\title{
Chemical and Nuclear Properties of Lawrencium (Element 103) and Hahnium (Element 105)
}

\author{
by \\ Roger Alan Henderson \\ Ph.D. Thesis \\ (September 10, 1990) \\ Department of Chemistry \\ University of California, Berkeley \\ and \\ Nuclear Science Division \\ Lawrence Berkeley Laboratory \\ 1 Cyclotron Road \\ Berkeley, CA 94720
}

This work was supported in part by the Director, Office of Basic Energy Sciences, Chemical Sciences Division of the U.S. Department of Energy under Contract No. DEAC03-76SF00098.

ivisol- 


\title{
Chemical and Nuclear Properties of Lawrencium (Element 103) and Hahnium (Element 105)
}

\author{
by
}

\section{Roger Alan Henderson}

\begin{abstract}
The chemical and nuclear properties of $\mathrm{Lr}$ and Ha have been studied, using 3-minute ${ }^{260} \mathrm{Lr}$ and 35 -second ${ }^{262} \mathrm{Ha}$. The crystal ionic radius of $\mathrm{Lr}^{3+}$ was determined by comparing its elution position from a cation-exchange resin column with those of lanthanide elements having known ionic radii. Comparisons are made to the ionic radii of the heavy actinides, $\mathrm{Am}^{3+}$ through $\mathrm{Es}^{3+}$, obtained by $\mathrm{x}$-ray diffraction methods, and to $\mathrm{Md}^{3+}$ and $\mathrm{Fm}^{3+}$ which were determined in the same manner as $\mathrm{Lr}^{3+}$. The hydration enthalpy of $-3622 \mathrm{~kJ} / \mathrm{mol}$ was calculated from the crystal ionic radius using an empirical form of the Born equation. Comparisons to the spacings between the ionic radii of the heaviest members of the lanthanicle series show that the $2 \mathrm{Z}$ spacing between $\mathrm{Lr}^{3+}$ and $\mathrm{Md}^{3+}$ is anomalously small, as the ionic radius of $\mathrm{Lr}^{3+}$ of $0.0886 \mathrm{~nm}$ is significantly smaller than had been expected.

The chemical properties of $\mathrm{Ha}$ were determined relative to the lighter homologs in group 5. Nb and Ta. Group 4 and group 5 tracer activities, as well as $\mathrm{Ha}$, were adsorbed onto glass surfaces as a first step toward the determination of the chemical properties of Ha. Ha was found to adsorb on surfaces, a chemical property unique to the group 5 elements, and as such demonstrates that $\mathrm{Ha}$ has the chemical properties of a group 5 element. A solvent extraction procedure was adapted for use as a micro-scale chemical procedure to examine whether or not Ha displays eka-Ta-like chemical under conditions where $\mathrm{Ta}$ will be extracted into the organic phase and $\mathrm{Nb}$ will not. Under the conditions of this experiment Ha did not extract, and does not show eka-Ta-like chemical properties.
\end{abstract}


Some nuclear properties of ${ }^{261} \mathrm{Rf}$ and ${ }^{261} \mathrm{Lr}$ were also studied. An electron-capture decay branch in ${ }^{261} \mathrm{Rf}$, produced by the ${ }^{248} \mathrm{Cm}\left({ }^{18} \mathrm{O}, 5 \mathrm{n}\right)$ reaction, was searched for by detecting the daughter activity, ${ }^{261} \mathrm{Lr}$. In addition, the half-life and fission properties of ${ }^{261} \mathrm{Lr}$, froduced by the ${ }^{248} \mathrm{Cm}\left({ }^{18} \mathrm{O}, \mathrm{p} 4 \mathrm{n}\right)$ reaction, were examined. The total kinetic energy of about $210 \mathrm{MeV}$ was compared to the total kinetic energies of other spontaneously-fissioning nuclides.

The production cross sections for ${ }^{260} \mathrm{Lr}$ from the ${ }^{249} \mathrm{Bk}\left({ }^{18} \mathrm{O}, \alpha 3 \mathrm{n}\right)$ reaction, ${ }^{261} \mathrm{Lr}$ and ${ }^{262} \mathrm{Lr}$ from the ${ }^{248} \mathrm{Cm}\left({ }^{18} \mathrm{O}, \mathrm{p} 4 \mathrm{n}\right)$ and ${ }^{248} \mathrm{Cm}\left({ }^{18} \mathrm{O}, \mathrm{p} 3 \mathrm{n}\right)$ reactions, were determined to be $8.7 \mathrm{nb}, 700 \mathrm{pb}$, and $240 \mathrm{pb}$, respectively. A comparison of the magnitudes of the (p4n) and $(\mathrm{p} 3 \mathrm{n})$ cross sections was used to set an upper limit of $14 \%$ on the EC branch in ${ }^{261} \mathrm{Rf}$. 


\section{Dedication:}

This thesis is dedicated to my high school chemistry and calculus teacher, Dr. Margaret Cox. She inspired me to pursue chemistry as my career. She made learning chemistry quite enjoyable through her presence and teaching ability. Her cheerful mode was always appreciated throughout the year of calculus, which normally is just another boring math class. 


\section{Acknowledgments:}

First I would like to thank my parents, Richard and Barbara, who have given me much needed and appreciated support throughout my academic career. I would also like to thank my sister, Mary Kay, for her support and encouragement over the years.

I wish to thank my advisor for the past six years, Dr. Darleane C. Hoffman, for her constant encouragement and support, for without it this would not be possible.

I also wish to thank the Hoffman group members Aaron, Andreas, Beth, Bob, Bob, Bobby, Carolyn, Chris, Diana, Dianne, Flipper, George, Howard, John, Ken, Lithium, Matti, Michelle, Nancy, Peter, Steve, Teresita, and Yuichi for friendship and helping to make my stay in Berkeley more enjoyable.

I am indebted to the staff and crew of the $88^{\prime \prime}$ Cyclotron for their excellent work in support of this project, providing the particle beams necessary to create the isotopes for analysis.

I am indebted to the Division of Chemical Sciences, Office of Basic Energy Sciences, U.S. Department of Energy, for making the ${ }^{248} \mathrm{Cm}$ and ${ }^{249} \mathrm{Bk}$ available through the transplutonium element production facilities at the Oak Ridge National Laboratory.

Last of all, I wish to give my brothers in Alpha Chi Sigma, especially the Sigma chapter, my heartfelt thanks for the friendship and support that I enjoyed while living in the chapter house.

This work was supported in part by the [irector, Office of Basic Energy Sciences, 
Chemical Sciences Division of the U.S. Department of Energy under Contract No. DEAC03-76SF00098. 


\section{Table of Contents:}

I. Introduction 1

A. History 4

II. Experimental Procedures

A. Targets and Target Systems 11

B. Production of Isotopes 17

C. Chemistry

C1. $\alpha$-HIB Separations 18

C2. ACCESS Automated $\alpha$-HIB 22

C3. ACCESS Chemistry with Catcher Foils 23

C4. Extractions 26

C5. Adsorption Procedure 29

D. Detector Systems 31

E. Data Analysis 34

III. Lawrencium Studies

A. Extraction Studies 35

B. Ion Exchange Studies 37

C. Production of ${ }^{261} \mathrm{Lr}$ 46

D. ${ }^{262} \mathrm{Lr}$ Experiments 52 
IV. Hahnium Experiments

A. Adsorption Studies

B. Extraction Studies

60

V. Discussion

A. Chemistry of Lawrencium 64

B. Isotopes of Lawrencium 66

C. Chemistry of Hahnium 67

D. Future 67

Appendix A. Attempts to Reduce $\mathrm{Lr}^{3+}$

A. Introduction 69

B. Chemistry 70

C. Results and Discussion of the HDEHP Experiment 71

D. Non-Aqueous Experiments 73

1. Column Procedure $-74$

2. Results and Discussion 75

Appendix B. Attempts to Measure the Ionic Radius of $\mathrm{No}^{3+}$

A. Introduction 77

B. Chemical Procedure 79

C. Results and Discussion 80

References 84 


\section{Foreword:}

This thesis contains material from these two published papers:

Atom-at-a-Time Radiochemical Separations of the Heaviest Elements; Lawrencium Chemistry, J. Radioanal. Nucl. Chem. 124, 135 (1988) by D.C. Hoffman, R.A. Henderson, K.E. Gregorich, D.A. Bennett, R.M. Chasteler, C.M. Gannett, H.L. Hall, D.M. Lee, M.J. Nurmia, S.Y. Cai, R. Agarwal, A.W. Charlop, Y.Y. Chu, and R.J. Silva

Aqueous Chemistry of Element 105, Radiochimica Acta 43, 233 (1988) by K.E. Gregorich, R.A. Henderson, D.M. Lee, M.J. Nurmia, R.M. Chasteler, H.L. Hall, D.A. Bennett, C.M. Gannett, R.B. Chadwick, J.D. Leyba, D.C. Hoffman, and G. Hermann 


\section{Chapter I: Introduction}

Very little is currently known about the chemical properties of the heaviest elements. It is of particular interest to compare the chemical properties of the elements at the end of the actinide series and the beginning of the transactinide series with those of their lighter homologs, the heavy lanthanides, and the group 4 and group 5 elements, respectively. It is of special interest to confirm Seaborg's actinide concept [SEA45] by studying the chemical and nuclear properties of element 103, lawrencium, at the end of the actinides, and of elements 104 and 105 at the beginning of the transactinides, in particular to see whether trends in chemical properties of a given group can be extrapolated to their heaviest members at the very end of the table. The heaviest members of the actinide series, starting with Am, are most stable in aqueous solution as $3+$ cations, with the exception of No, which is most stable in the $2+$ oxidation state. Nobelium can exist in the $2+$ oxidation state in aqueous solution because this results in the stable electronic configuration $[\mathrm{Xe}] 5 \mathrm{f}^{14}$, with the completed $5 \mathrm{f}$ shell. Nobelium can be oxidized to the $3+$ state using $\mathrm{H}_{5} \mathrm{IO}_{6}$ which has a standard reduction potential, $\mathrm{E}^{\mathrm{O}}=+1.7 \mathrm{~V}$ [SIL69]. Another characteristic chemical property of the $3+$ actinide ions is the formation of sesquioxides of the form $\mathrm{M}_{2} \mathrm{O}_{3}$. Tetravalent oxidation states are known in some of the heavier actinides, $\mathrm{Am}, \mathrm{Cm}, \mathrm{Bk}$, and $\mathrm{Cf}$. As $\mathrm{Z}$ increases, it becomes more difficult to oxidize the element to the $4+$ oxidation state. $2+$ oxidation states can also be formed among many of the heavy actinides; however, they are typically unstable in aqueous solution.

Few chemical experiments on the transactinide elements have been performed. One question of interest in this region is whether or not the early transactinide elements will begin a new $6 \mathrm{~d}$ series, similar to those started by Hf and Ta, or whether the chemical properties will be changed by Relativistic effects arising from the very high nuclear charge. Of these elements, the chemical properties of only Rf and Ha have been studied. 
The experiments on $\mathrm{Ha}$ will be discussed later. For Rf, previous experiments have shown [SIL70A] that the stable oxidation state of $\mathrm{Rf}$ is $4+$ in aqueous solution, and that its properties place it in Group 4. Gas phase experiments have also been performed [ZVA66][ZVA69][ZVA70], with the result that $\mathrm{Rf}$ has been shown to form a tetravalent chloride complex as Hf does, and is, therefore, a member of Group 4 in the periodic system.

The heaviest elements are characterized by their short half-lives. The isotopes of these elements decay by all known decay modes. However, alpha decay and spontaneous fission (SF) are the most prominent modes. Beta decay, both $\mathrm{B}^{-}$and electron capture, is also exhibited. Shell effects and unpaired nucleons play a very large role in the stability of these elements. The shell effects are responsible for the existence of the elements heavier than fermium. The liquid-drop model fission barriers in this region are small at best, and for the elements beyond Fm, the fission barrier is predicted to be zero by this model. The observation of fission isorners led to the development of the Strutinsky method of shell corrections to the liquid drop model [STR67]. The major result of this theory is the explanation of shell stabilization towards fission, and the existence of fission isomers as a consequence of a double-humped fission barrier. Isomeric states are also known to exist in many isotopes that have an odd neutron, odd proton, or both. Odd particles also stabilize an isotopes ciecay via SF [HOF87A].

The short half-lives of these elements make chemical experiments very difficult to perform, and often novel techniques must be developed and used. To effectively study the chemistry of these elements it is necessary to have procedures that can be carried out in a time comparable to the half-life, or less, of the isotope that is being used for study. The sample must also be in a suitable form for radiation measurements after the completion of a procedure. This requirement puts particular constraints on the type of procedures that can be performed for the heaviest element isotopes which often decay primarily by alpha or SF. For alpha detection, the samples must be as clean as possible, 
for any amount of mass that is present will degrade the resolution of the alpha spectrum, which can make positive identification of these isotopes quite difficult. Also, none of the isotopes that are used for study exist in weighable quantities, and often only a few atoms or a single atom are present at any one time. This small amount of material makes it nearly impossible to use such classical analytical techniques as $\mathrm{X}$-ray diffraction, polarography, and nearly all forms of optical spectroscopy, to name but a few. The procedure used must, therefore, put these few atoms through as many reactions as possible, so that statistically meaningful answers can be arrived at from these ultra-tracer scale experiments [BOR81][HUL83][KEL77]. Typically, thermochromatography, liquidliquid extractions, and ion exchange resin procedures satisfy these requirements. The experimental procedures used must often be repeated many times.

Comparison of the chemical properties of one group of elements to their lighter homologs is a very powerful technique for understanding the chemical properties of an unknown species. Elutions from cation-exchange resin columns can be performed using trace amounts of lanthanide elements to infer the behavior of the heaviest actinide elements. By comparing elution positions of a new element with those of elements of known properties, one can learn many things about the chemistry of the new element: for example, its ionic radius and the type of chemical complex that it has formed. Solvent extraction techniques can also be used in a similar fashion, and are another procedure that can be carried out quite rapidly.

The current research is involved with the determination of the fundamental chemical properties of $\mathrm{Lr}$ and $\mathrm{Ha}$. It is also concerned with finding new ways to produce isotopes of $\mathrm{Lr}$ for future chemical study, and also with determining the nuclear properties of some of these isotopes. Our research group has specialized in the development of very rapid micro-scale chemical procedures. Our access to heavy target materials, such as ${ }^{249} \mathrm{Bk}$ and ${ }^{248} \mathrm{Cm}$, and the hign-intensity beams that the 88 -Inch Cyclotron can deliver give us a unique opportunity for the study of the chemical properties of these short-lived isotopes 
of the heaviest elements.

\section{A. History}

Element 103, lawrencium, was discovered in 1961 by Ghiorso and co-workers [GHI61]. They produced a new 8.6-MeV alpha-emitting isotope with a $8 \pm 2$ second halflife in the reactions of ${ }^{11} \mathrm{~B}$ and ${ }^{10} \mathrm{~B}$ with ${ }^{252} \mathrm{Cf}$. This isotope was later assigned to ${ }^{258} \mathrm{Lr}$. Since that time, a total of ten isotopes have been produced and identified, (See Table I). The isotope with the longest half-lire is ${ }^{262} \mathrm{Lr}$, which has a 3.6 hour half-life. Table I 1usts the currently known isotopes of $\mathrm{Lr}$.

\section{Table I: Isotopes of Lawrencium}

\begin{tabular}{|c|c|c|c|}
\hline Mass Number & Half-life & $\begin{array}{c}\text { Decay Modes anu } \\
\alpha \text { Energies (MeV) }\end{array}$ & References \\
\hline 253 & $1.3 \mathrm{~s}$ & $\alpha 8.800,8.722$ & [HES85] \\
\hline 254 & $13 \mathrm{~s}$ & $\alpha 8.460,8.403$ & [MUN81][HES85] \\
\hline 255 & $22 \mathrm{~s}$ & $\alpha 8.410,8.360$ & [BEM76][ESK71] \\
\hline 256 & $28 \mathrm{~s}$ & $\begin{array}{c}\alpha 8.635,8.520, \\
8.475,8.430 \\
8.290,8.319\end{array}$ & $\begin{array}{c}\text { [DON65][ESK71] } \\
\text { [BEM76] }\end{array}$ \\
\hline 257 & $0.646 \mathrm{~s}$ & $\alpha 8.864,8.800$ & [ESK71][BEM76] \\
\hline 258 & $4.3 \mathrm{~s}$ & $\begin{array}{c}\alpha 8.654,8.621 \\
8.595,8.565\end{array}$ & [ESK71][BEM76] \\
\hline 259 & $5.4 \mathrm{~s}$ & $\alpha 8.450$ & {$[$ [ESK71] } \\
\hline 260 & $3.0 \mathrm{~m}$ & $\alpha 8.030$ & {$[$ [ESK71] } \\
\hline 261 & $39 \mathrm{~m}$ & $S F, E C ?$ & {$[$ [LOU87] } \\
\hline 262 & $3.6 \mathrm{~h}$ & $\alpha \mathrm{EC}$ & [LOU87] \\
\hline
\end{tabular}


The first chemical study of $\mathrm{Lr}$ was reported in 1970 by Silva and co-workers [SIL70]. They studied the extraction of $25.9-\mathrm{s}{ }^{256} \mathrm{Lr}$ with thenoyltrifluoroacetone, TTA, dissolved in methylisobutyl ketone, MIBK. Buffered acetic and monochloroacetic acids served as the aqueous phases. The extraction behavior of $\mathrm{Lr}$ was compared directly to the extraction properties of other $3+$ actinide ions, and was found to be quite similar, showing that $\mathrm{Lr}$ exists in the $3+$ oxidation state in aqueous solution. Element 104 was found to be tetravalent [SIL70A] thus confirming that $\mathrm{Lr}$ completes the 14 member actinide series, as Seaborg had predicted in 1945 [SEA45]. Until the present study, no further experiments had been performed to determine other chemical properties of $\mathrm{Lr}$.

Element 105 , hahnium, has a history clouded with political debate over the conflicting claims as to its criginal discovery. Soviet scientists under G.N. Flerov [FLE68] have claimed the initial discovery and have proposed the name niels bohrium. American scientists led by A. Ghiorso [GHI70] have also claimed the initial discovery, and have proposed the name used in this paper, hahnium. A detailed discussion of the controversy concerning the discovery has been given by Hyde, Hoffman, and Keller [HYYD87]. Following the discovery experiments, a total of five more isotopes of Ha have been discovered, with the longest-lived isotope being 40-sec. ${ }^{262} \mathrm{Ha}$. Table II lists the currenti' ${ }^{\prime}$ known isotopes of $\mathrm{Ha}$.

Following the discoveries of element 105 reported in 1968 and 1970, respectively, only two gas-phase experiments by Zvara [ZVA74],[ZVA76] and no aqueous phase experiments have

been performed to determine chemical properties of Ha. These experiments were designed to look at the volatile pentachloride and pentabromide species. The adsorption characteristics of volatile compounds formed from interactions with different halogenating species were studied. These experiments were nterpreted as showing that $\mathrm{Ha}$, presumed to exist as volatile $\mathrm{HaBr}_{5}$ or $\mathrm{HaCl}_{5}$ species, had adsorption characteristics similur to $\mathrm{TaBr}_{5}$ or $\mathrm{TaCl}_{5}$. As is the case with $\mathrm{Lr}$, no further studies of the chemical 
properties of $\mathrm{Ha}$ have been attempted until the current study.

\section{Table Il: Isotopes of Hahnium}

\begin{tabular}{|c|c|c|c|}
\hline Mass Number & Half-life & $\begin{array}{l}\text { Decay Modes and } \\
\alpha \text { Energies }(\mathrm{MeV})\end{array}$ & References \\
\hline 255 & $\cong 1.2 \mathrm{~s}$ & $\mathrm{SF} \cong 20 \%$ (est.) & [OGA76][FLE76] \\
\hline 256 & $2.6 \mathrm{~s}$ & $? ? ?$ & [OGA83] \\
\hline 257 & $1.4 \mathrm{~s}$ & $\begin{array}{l}\alpha 9.160,9.071, \\
8.970 ; \text { SF }(17 \%)\end{array}$ & [OGA76][HES85] \\
\hline 258 & $4.4 \mathrm{~s}$ & $\begin{array}{c}\alpha 9.299,9.172 \\
9.078,9.008 \\
\text { EC }(33 \%)\end{array}$ & [HES85] \\
\hline 260 & $1.52 \mathrm{~s}$ & $\begin{array}{c}\alpha 9.128,9.082 \\
9.047 ; \operatorname{SF}(<9.6 \%)\end{array}$ & [BEM77][GHI70] \\
\hline 261 & $1.8 \mathrm{~s}$ & $\alpha 8.93 ; \mathrm{SF}(\cong 25 \%)$ & [GHI71][FLE70] \\
\hline 262 & $34 \mathrm{~s}$ & $\begin{array}{c}\alpha 8.670,8.530 \\
8.450 ; \text { SF }(71 \%) ; \\
\text { EC }(\cong 3 \%)\end{array}$ & [GHI71][BEM7TA] \\
\hline
\end{tabular}

Continued study of the chemistry of these two elements is of interest for several reasons. The architecture of the periodic table is used to help predict the chemical properties of elements in a homologous series. It is indeed very interesting to see if the trends observed in a given group of the periodic table can be extrapolated down to the heaviest members of a group. Any changes in these trends in the heavy element region are of key interest. 
A basic understanding of the chemical properties of an element can be achieved through thermodynamic considerations. One item of particular interest is the heat of formation of the aqueous $3+$ ions which are formed throughout the lanthanide series and the latter half of the actinide series. The heat of formation can be broken down into its substituent components through the use of the Born-Haber cycle.

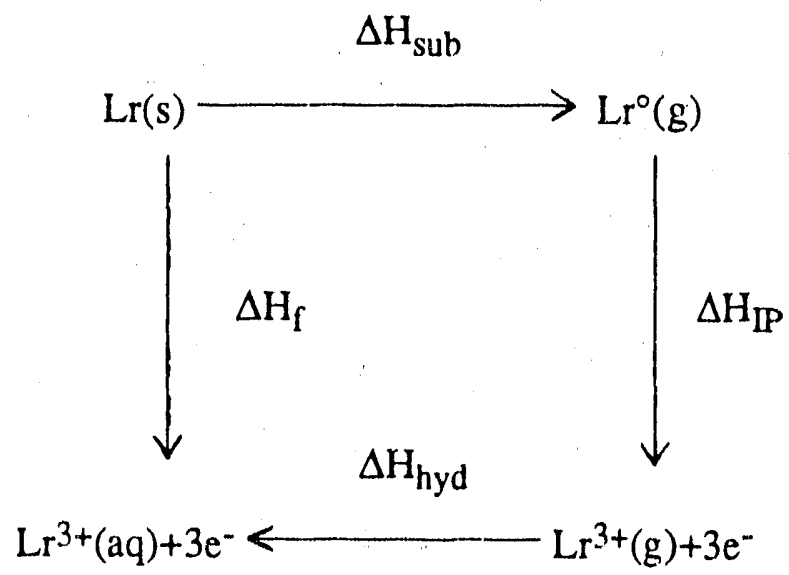

The four separate components can be determined as follows. The heat of sublimation can be determined through thermochromatographic means, similar to those that were used for the study of $\mathrm{Ha}$ gas phase chemistry. The heat of ionization can be calculated only with the use of highly sophisticated relativistic Dirac-Fock computer codes. The hydration cnthalpy can be calculated from an empirical form of the Born equation, for example, by use of eq. 1 [PHI66]. In this equation, $z$ is the charge of the ion and $r$ is the Pauling crystal ionic radius in $\mathrm{nm}$. The heat of formation can be determined in the actinire case by the use of radiopolarography. However, this technique has not been developed sufficiently to measure properties on the few-atom scale that would be necessary for elements 103 and 105. In the case of the hydration enthalpy, one notes that this quantity can be calculated from the ionic radius as follows:

$$
\Delta \mathrm{H}_{\text {hyd }}=-69.9 \mathrm{z}^{2} /(\mathrm{r}+0.085) \mathrm{kJ} / \mathrm{mol} \quad \text { eq. } 1
$$


Marcus and Kertes [MAR69] have pointed out that in ion exchange processes the distribution coefficient $\mathrm{Ha}$ a linear relationship to the ionic radius. By comparing the elution position of ar, unknown ion to the elution positions of ions with similar coordination numbers and known ionic radii, one can then infer the ionic radius for the unknown ion. Ion exchange procedures appropriate to this problem have been in existence for some time. In particular, cation exchange using the eluting agent alphahydroxyisobutyrate, $\alpha$-HIB, provides an excellent method for studying the ionic radius of $\mathrm{Lr}^{3+}$. Studies by Smith and Hoffman [SMI56] and by Choppin and Silva [CHO56] have shown that a linear relationstip betwien the elution position and the crystal ionic radius does indeed exist for the lanthanides and the 3+ actinide elements. By directly comparing the elution position of $\mathrm{Lr}^{3+}$ with some lanthanide tracers, it is possible to infer a value for the ion $c$ radius, thus allowing a vaitie for the hydration enthalpy to be calculated.

Different interpretations of the results of Zvara on the gas-phase experiments with $\mathrm{Ha}$ have been offered by Keller and Seaborg[KEL77] and by Hyde et.al.[HYD87]. Their interpretations of the data leave some doub: as to whether or not the chemical similarity of $\mathrm{Ha}$ to its homologs $\mathrm{Nb}$ and $\mathrm{Ta}$ has been demonstrated. Differences between the chemistry of $\mathrm{Ha}$ and its horwologs can also be explored using rapid aqueous chemical procedures. The best procedures offering the quickest processing times are single-step solvent extractions. By comparing the ex'ractability of Ha to its homologs in different systems, one can determine how well the periodic trends in the lighter elements extrapolate to the heavy element region.

Relativistic effects may play a large role in determining the chemical properties of the heaviest elements. These relativistic effects are mainly manifested as increased binding of the low angular momentum quantum number electrons, the $\mathrm{s}$ and $\mathrm{p}$ electrons. Increased binding energy is an example of a direct relativistic effect. There are also indirect relativistic effects, in that electrons in higher angular momentum orbitals will see less of the nuclear charge due to increased screening by the $s$ and $p$ electrons. Hence 
they will have larger radii and lower binding energies than without relativistic effects [KEL84][PIT79][PYY79][PYY88]. These effects may stabilize lower oxidation states of heavy actinides relative to the homologous lanthanide elements, and may also have a noticeable effect on the size of the atom or its ion(s). Attempts to look for a relativistically stabilized $1+$ oxidation state in $\mathrm{Lr}$ are described in .Appendix A.

Another manifestation of relativistic effec $\iota s$ is the charige in the order of electron shell filling when compared to the lighter homologs of the periodic table. $\mathrm{Lr}$, by analogy to $\mathrm{Lu}$, should have the ground-state electronic configuration of $[R n] 5 f^{14} 6 d^{1} 7 s^{2}$. However, relativistic calculations by Brewer [BRE84], Desclaux and Fricke [DES80], and Pyykko [PYY88] for the neutral atom suggest that the ground state should be $[R n] 5 f^{14} 7 s^{2} 7 p_{1 / 2}$. Experimental evidence for this does not exist as yet. However, methods to prove this using the Stern-Gerlach technique have been proposed [HUL84]. Few direct comparisons of non-relativistic and relativistically adjusted electronic configurations exist; however, multiconfiguration Dirac-Fock (MCDF) calculations by Glebov et.al. [GLE89] have shown that relativistic effects in element 104 , Rf, play a major role in determining the [RN] $5 p^{14} 6 \mathrm{~d} 7 \mathrm{~s}^{2} 7 p_{1 / 2}$ atomic ground state, and find that the $6 \mathrm{~d}^{27} \mathrm{~s}^{2}$ state is only $0.6 \mathrm{eV}$ above the ground state.

Special facilities and expertise are essential if these heaviest elements are to be studied. The 88" Cyclotron at the Lawrence Berkeley Laboratory provides high intensity beams of light heavy ions for the bombardment of heavy actinide targets for the production of isotopes for study. Target materials of ${ }^{248} \mathrm{Cm}$ and ${ }^{249} \mathrm{Bk}$ are produced at the High Flux Isotope Reactor, HFIR; at the Oak Ridge National Laboratory. $\mathrm{He} / \mathrm{KCl}$ gas jet transport systems facilitate the removal of the reaction products from the target chamber to remote chemical processing sites. The development of specialized microscale chemical techniques has also helped to make the study of the heaviest elements possible. These techniques allow the separation of the desired element from the large quantities of reaction products that result from beam interactions with the target as well 
as the fission products from the target material.

Since the HFIR reactor did not operate for several years during the course of this research, methods for producing isotopes of $\mathrm{Lr}$ that do not rely on using the relatively short-lived ${ }^{249} \mathrm{Bk}$ (325-d) or ${ }^{254} \mathrm{Es}$ (275-d) target materials were investigated. Production methods using lighter target material, e.g. ${ }^{248} \mathrm{Cm}\left(3.397 \times 10^{5}-\mathrm{y}\right)$, that does not decay rapidly and is readily available were explored. Production methods using the decay of heavier isotopes into the longer-lived $\mathrm{Lr}$ isotopes also hold promise for future chemical studies of Lr. 


\section{Chapter II: Experimental Procedures}

\section{A. 'Targets and Target Systems}

All irradiations were carried out at the Lawrence Berkeley Laboratory's 88" Cyclotron. Targets consisting of either ${ }^{249} \mathrm{Bk}$ or ${ }^{248} \mathrm{Cm}$ sesquioxides were prepared by electrodepositing the respective $\mathrm{M}\left(\mathrm{NO}_{3}\right)_{3}$ species by a molecular deposition technique [AUM74][EVA72][MUL75] from an isopropanol solution. The deposited $\mathrm{NO}_{3}^{-}$species were converted to the oxide form by heating in an oven for thirty minutes at $450-500^{\circ} \mathrm{C}$. This method of target preparation has been shown [MOO83] to give superior targets for irradiation. This method produces uniform targets that adhere well to the substrate, resisting flaking that can occur with targets that are evaporated onto the backing.

325 -day ${ }^{249} \mathrm{Bk}$ was purified from its beta-decay daughter ${ }^{249} \mathrm{Cf}$ by oxidation of the $\mathrm{Bk}^{3+}$ to the $4+$ oxidation state using saturated $\mathrm{NaBrC}_{3}$. The $\mathrm{Bk}^{4+}$ was then extracted into a solution of $0.5 \mathrm{M}$ HDEHP (di-2-ethylhexylorthophosphoric acid) in heptane [HOR69A], and back extracted with $3 \mathrm{M} \mathrm{HCl}$ solution containing a small amount of $\mathrm{H}_{2} \mathrm{O}_{2}$ to reduce $\mathrm{Bk}^{4+}$ to $\mathrm{Bk}^{3+}$. The resulting solution was taken to dryness under a heat lamp. The $\mathrm{BkCl}_{3}$ was converted to the nitrate form by evaporating to dryness several times from a $2 \mathrm{M} \mathrm{HNO}_{3}$ acid solution. The resulting $\mathrm{NO}_{3}{ }^{-}$salt was dissolved in $2 \mathrm{ml}$ of pure isopropanol and placed in an electroplating cell. A diagram of the electroplating apparatus is shown in figure 1. A layer of approximately $50 \mu \mathrm{g} / \mathrm{cm}^{2}$ was deposited on a Be foil in a $6-\mathrm{mm}$ diameter circle, as defined by the chimney of the plating apparatus. Deposition is carried out by applying a voltage of several hundred volts. The solution is stirred during the electroplating by attaching the plating electrode to the center of a small speaker. The plating power supply is adjusted so that the current does not exceed 6 $\mathrm{mA} / \mathrm{cm}^{2}$, at which point the solution will begin to bubble and poor deposition will result. After deposition of each layer, the target was dried under a heat lamp, then baked in a 


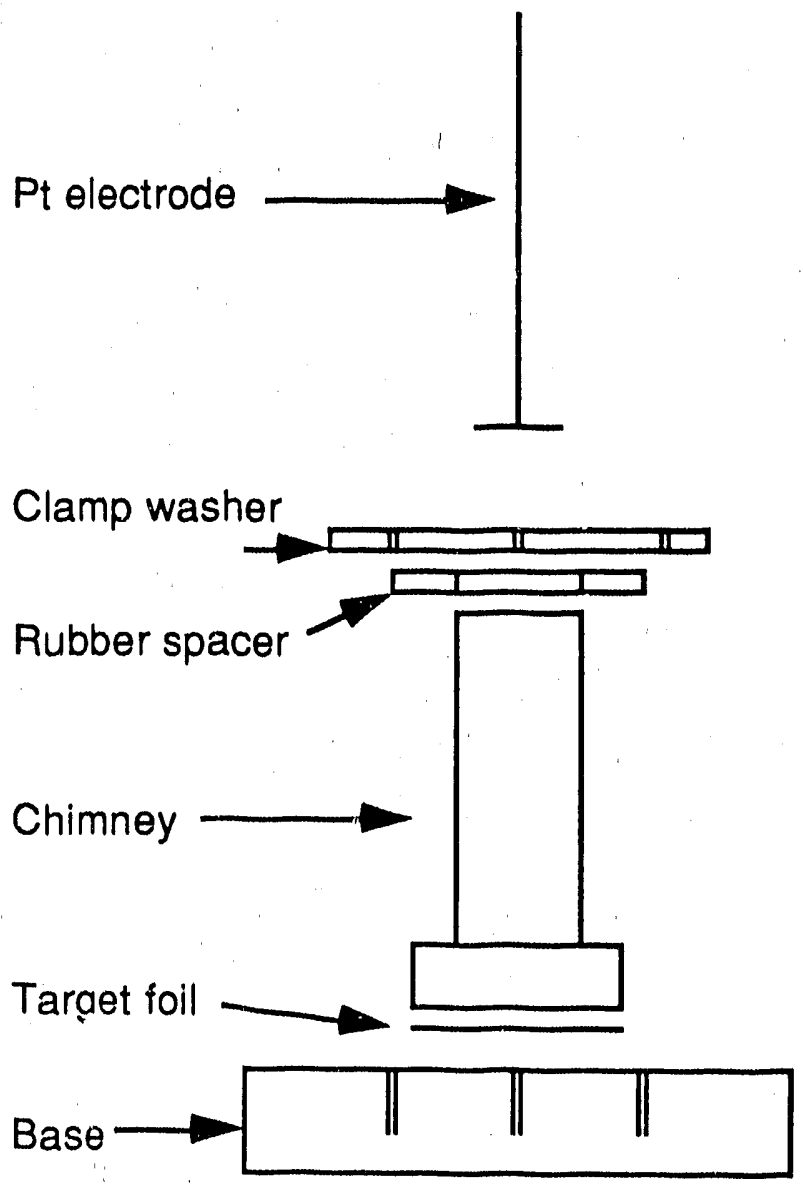

Figure 1: Electroplating cell 
$450-500^{\circ} \mathrm{C}$ oven for about thirty minutes. The process was repeated until the desired thickness was achieved. Assuming uniform deposition and a composition of ${ }^{249} \mathrm{Bk}_{2} \mathrm{O}_{3}$, the target thickness was determined by counting the alpha emission from the ${ }^{249} \mathrm{Bk}$ target material in a low geometry alpha chamber, utilizing the $5.4168-\mathrm{MeV}$ alpha particle for the determination. The efficiency of the detector has been determined to be $1.446 \times 10^{-4}$. The final thickness of this target was $940 \mu \mathrm{g} / \mathrm{cm}^{2}$.

${ }^{248} \mathrm{Cm}$ was purified by elution from a anion exchange resin column using conc. $\mathrm{HCl}$, which effects the separation of the $\mathrm{Cm}$ from its daughter nuclide, ${ }^{244} \mathrm{Pu}$. The final target was then prepared in the same manner as the Bk target. The final thickneiss of this target was $742 \mu \mathrm{g} / \mathrm{cm}^{2}$.

A schematic of the target system is shown in figure 2 . The ${ }^{18} \mathrm{O}^{5+}$ beam first enters a graphite collimator which defines the beam spot to be no larger than the area of the target material. The i.d. of the collimator was $6 \mathrm{~mm}$. The beam then passes through a Havar vacuum isolation.ndow, which separates the target system from the cyclotron vacuum. A small volume oi $\mathrm{N}_{2}$ gas is next, whose purpose is to cool the target backing and target material, and also the Havar vacuum isolation foil. The beam then passes through the $\mathrm{Be}$ target backing, spaced $2 \mathrm{~mm}$ from the isolation foil, followed by the target material. The beam energies on target were calculated using the range tables of Northcliff and Schilling [NOR70]. The beam is stopped in a 1-cm thick graphite block mounted on the back plate of the target chamber, $15 \mathrm{~cm}$ from the target. The back plate of the target chamber as well as the collimator flange are water cooled to prevent excessive heat buildup from the beam.

For some of the experiments, the Light Ion Multiple target system, LIM, was used [HAL89] with two ${ }^{248} \mathrm{Cm}$ targets. This use of multiple targets allows the production rate to increase with the number of targets being used. Figure 3 shows a schematic of the LIM target system as it was used in these experiments. The first target, the same $742 \mu \mathrm{g} / \mathrm{cm}^{2}$ ${ }^{248} \mathrm{Cm}$ target mentioned above, is mounted in the normal position inside the target 

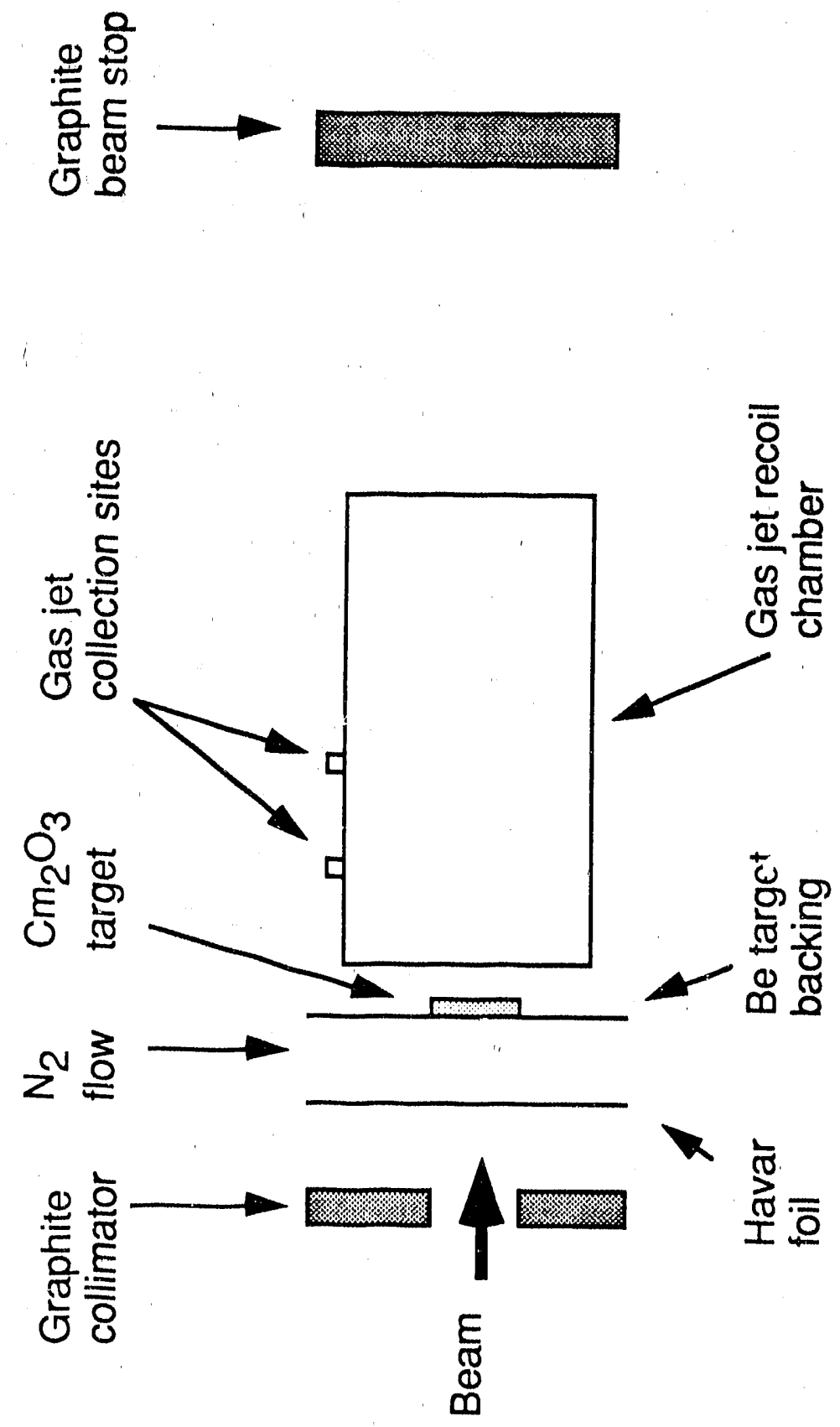

Figure 2: Target system schematic 


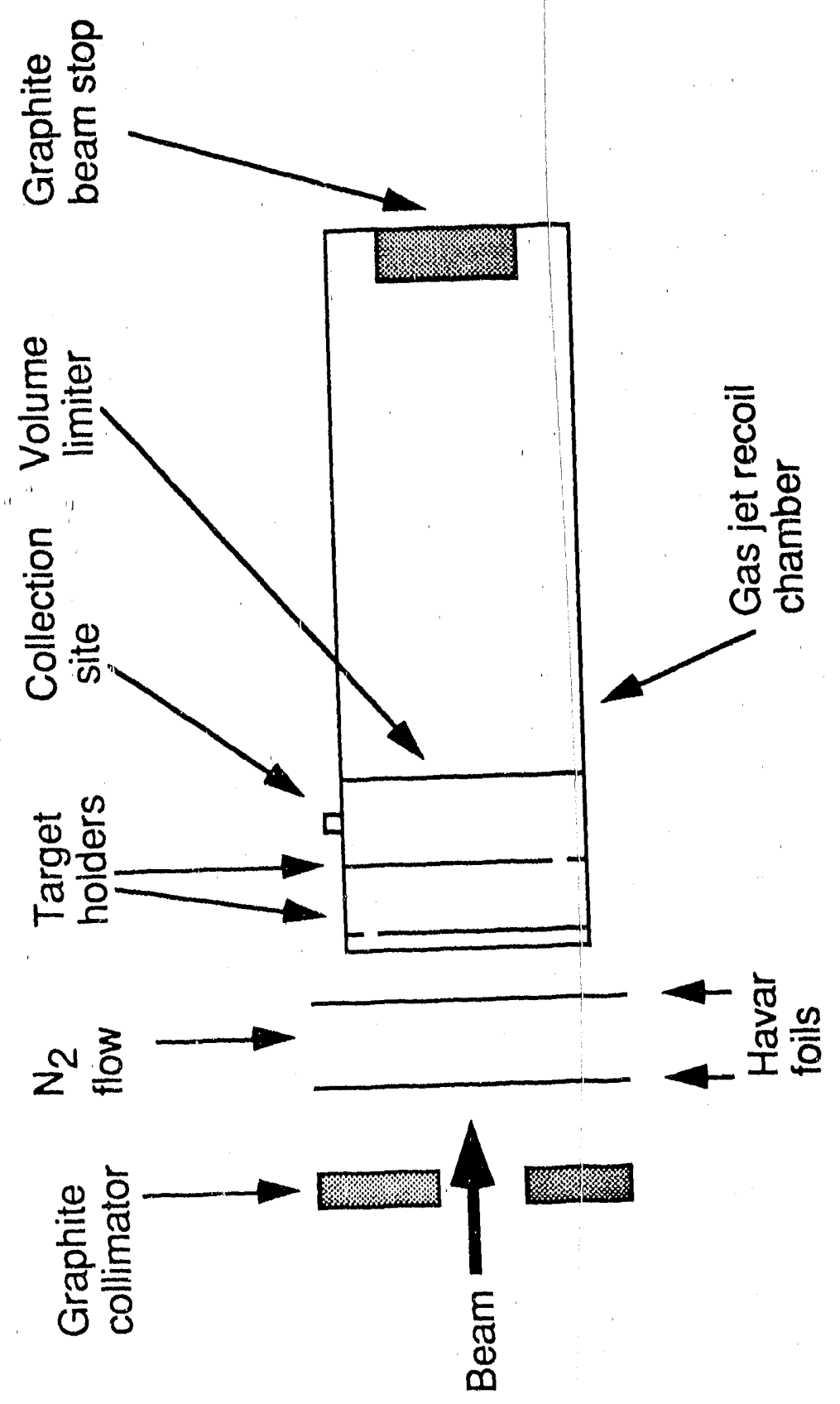

Figure 3: Light Ion Multiple Target (LIM) system schematic 
chamber. The second target, a $491 \mu \mathrm{g} / \mathrm{cm}^{2}{ }^{248} \mathrm{Cm}$ target, was placed in the LIM target stack holder. This second target was placed so that it was at about two and a half times, about $4 \mathrm{~cm}$, the recoil range of the compound-nucleus products in the $\mathrm{He}$ inside the chamber. A Be volume limiting foil is placed $5 \mathrm{~cm}$ behind the second target inside the target stack to confine the $\mathrm{He} / \mathrm{KCl}$ aerosols to a small volume directly after the second target. Milled slots around the second target allow the $\mathrm{He} / \mathrm{KCl}$ transport system to collect products from the first target before sweeping out the volume behind the second target. The He pressure in the recoil chamber was adjusted to about $1.2 \mathrm{~atm}$. The compound nucleus range in $\mathrm{He}$ for an incident $98-\mathrm{MeV}{ }^{18} \mathrm{O}$ projectile on a ${ }^{248} \mathrm{Cm}$ target is 0.3 $\mathrm{mg} / \mathrm{cm}^{2}$, or $1.5 \mathrm{~cm}$. Transport of the reaction products from the LIM system is achieved by means of the $\mathrm{He} / \mathrm{KCl}$ aerosol jet transport system.

Depending on the needs of the experiment, the recoiling product atoms are either caught in a Au catcher foil that is placed directly behind the target, about $2 \mathrm{~mm}$ away, or attached to $\mathrm{KCl}$ aerosol particles and swept out of the target chamber through a $1.5-\mathrm{mm}$ i.d. capillary tube to a remote site for chemical processing.

The $\mathrm{KCl}$ aerosols for the $\mathrm{He} / \mathrm{KCl}$ gas jet system were generated by heating crystalline $\mathrm{KCl}$ to $690^{\circ} \mathrm{C}$ inside a quartz tube. Helium gas was used to sweep the aerosols out of the tube and into a 'presorter' capillary, where the larger aerosol particles were allowed to settle out of the He flow. The presorter is a 5 meter length of 2-mm i.d. polypropylene capillary tube which has been wound into several coils of about $15 \mathrm{~cm}$ diameter. The 'presorted' aerosols were transported directly into the target chamber via a 3/8" i.d. tygon tube. Once inside the target chamber the aerosols were confined to a small volume directly behind the target, as shown in figure 2 . They were then taken from the target system via a small capillary tube, $1.5-\mathrm{mm}$ i.d., to a remote collection site on top of the experimental cave. The length of the capillary tube was about 7 meters. The aerosol transport system has two positions inside the recoil chamber for collection of the aerosols from single target experiments. One collection site is $2 \mathrm{~cm}$ from the plane of the target, 
the second is $4.5 \mathrm{~cm}$ away. The collection efficiency was found to be the best from the closest collection site. For the experiments performed with the LIM system, the collection site is on the target stack holder, immediately in front of the volume limiting Be foil.

The exterior collection site was inside a small lucite box placed in one corner of a fume hood, figure 4 . The aerosols were collected from the end of the capillary onto a surface, usually a $\mathrm{Pt}$ foil, held under vacuum. Four collection sites were placed around the circumference of a brass turntable. The vacuum was controlled by using an electric solenoid valve. A micro-switch at the edge of the wheel indicated when the wheel was moved. The signal from the switch was fed into the Realtime data Acquisition and Graphics System, RAGS, providing the start and stop times for the collections. RAGS recorded the beam integral during the experiments, as well as all of the alpha and fission data, which includes event-by-event information, i.e. the time of the event, the detector number, and the pulse-height of the event.

\section{B. Production of Isotopes}

Isotopes of $\mathrm{Lr}$ were produced using both ${ }^{249} \mathrm{Bk}$ and ${ }^{248} \mathrm{Cm}$ targets. ${ }^{260} \mathrm{Lr}$ was produced by the ${ }^{249} \mathrm{Bk}(18 \mathrm{O}, \alpha 3 \mathrm{n})$ reaction. The cross section for this reaction was determined in this study. ${ }^{261} \mathrm{Lr}$ and ${ }^{262} \mathrm{Lr}$ were produced by interactions of ${ }^{18} \mathrm{O}$ with a ${ }^{248} \mathrm{Cm}$ target. These production reactions and cross sections are studied in this work.

${ }^{262} \mathrm{Ha}$ was produced by the ${ }^{249} \mathrm{Bk}\left({ }^{18} \mathrm{O}, 5 \mathrm{n}\right)$ reaction. The cross section for this reaction has been inferred to be $5 \mathrm{nb}$ by examination of the data from the discovery experiment [GHI71]. 


\section{Chemistry}

\section{C1. $\alpha$-HIB Separations}

The complexing agent alpha-hydroxyisobutyrate, $\alpha$-HIB, when used as an eluant for cations from a cation-exchange resin column, provides an excellent way to separate the actinide and lanthanide ions according to their ionic radii [SMI56][CHO56]. Depending upon the concentration and $\mathrm{pH}$ of the $\alpha$-HIB solution, the separation can be tailored to meet the specific requirements of the separation. The separation requirements for these studies were that $\mathrm{Lr}$ must be completely separated from $\mathrm{Md}$ so that the $\mathrm{Lr}$ isotopes used for these studies will not be confused with Md isotopes having similar decay properties. It is also necessary to perform the separation as rapidly as possible for the studies using ${ }^{260} \mathrm{Lr}$, as this isotope has only a three minute half-life. These seemingly incompatible requirements were both met with a manual procedure. The column used for this study consisted of a 2-mm i.d. x 20-mm Pt tipped glass column packed with Dionex cationexchange resin, $15 \mu \mathrm{m}$ particle size, to a height of $4 \mathrm{~cm}$. This is a pellicular type resin, with an active surface and an inert core. This type of resin has the advantage that it equilibrates much faster than por. Is or macro-porous resin types, allowing the separation to be carried out rapidly at room temperature, since it reaches equilibrium quickly. Prior to the on-line experimerts with $\mathrm{Lr}$, the column was carefully calibrated using lanthanide tracers to check the elution positions and the reproducibility of the separation.

$\mathrm{Ho}, \mathrm{Tm}, \mathrm{Er}$, and $\mathrm{Yb}$ tracer activities produced via neutron capture in a Triga Mark III researah reactor at the University of California, Berkeley, were used for the column calibrations. Small samples of the stable isotopes of each element were placed inside quartz tubes which were then sealed and placed into the central thimble of the reactor. They were then sıbjected to a neutron flux of $5 \times 10^{12} \mathrm{n} / \mathrm{cm}^{2}$.sec. The samples were left in the central thimble for about an hour. The central thimble region of the reactor provides 


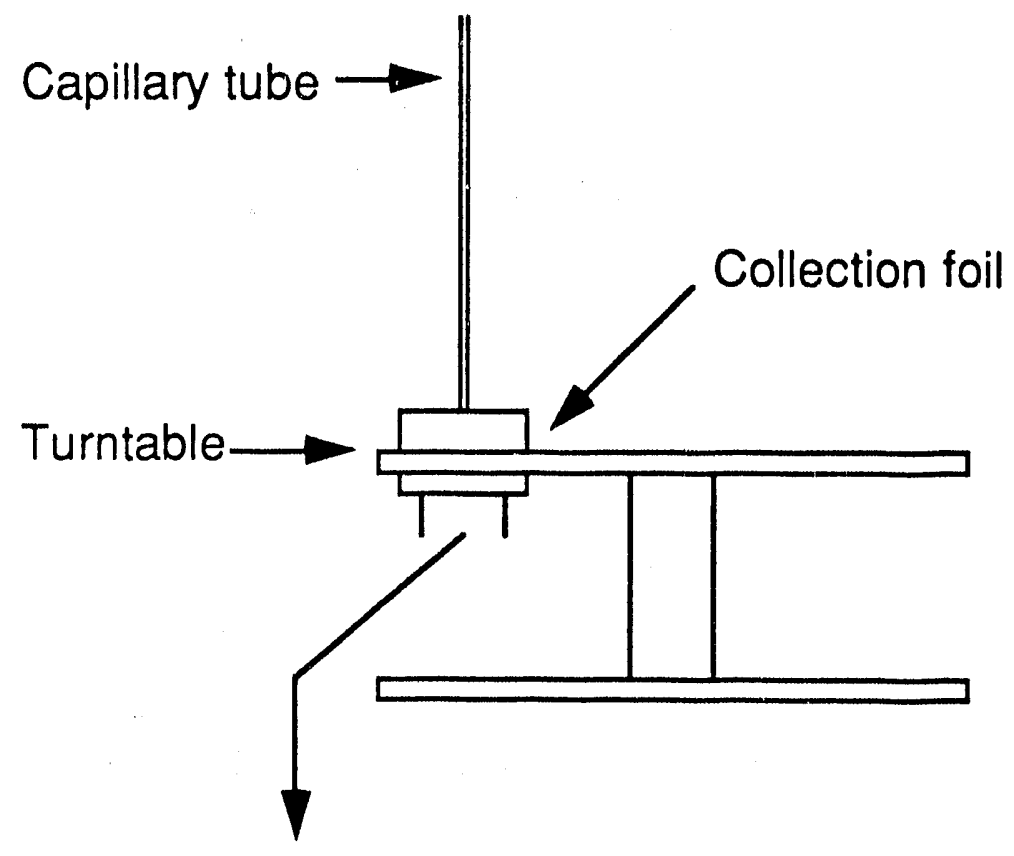

To vacuum pump

Figure 4: Gas jet collection site schematic 
the highest neutron flux, so that the specific activity of the tracers will be as high as possible. The isotopes that were predominantly formed and used in the $\alpha-H I B$ experiments were ${ }^{166} \mathrm{Ho},{ }^{171} \mathrm{Er},{ }^{170} \mathrm{Tm}$, and ${ }^{175} \mathrm{Yb}$.

Prior to each experiment the resin is converted to the $\mathrm{NH}_{4}+$ form by running a solution of $1 \mathrm{M} \mathrm{NH} \mathrm{NH}_{4} \mathrm{Cl}$ through the column. Reaction products recoiling from the target are removed from the target chamber using the $\mathrm{He} / \mathrm{KCl}$ aerosol jet transport system. The aerosols are then collected on a $\mathrm{Pt}$ foil. The $\mathrm{KCl}$ spot is dissolved using $50 \mu 1$ of $0.5 \mathrm{M}$ $\mathrm{HCl}$ containing the lanthanide tracers ${ }^{175} \mathrm{Yb}$ and $169,171 \mathrm{Er}$. The solution is pushed onto the resin bed using $\mathrm{N}_{2}$ gas pressure. The resin bed is then rinsed with $100 \mu \mathrm{l}$ of $\mathrm{H}_{2} \mathrm{O}$ to remove any remaining $\mathrm{HCl}$. Because separations using $\alpha$-HIB are very $\mathrm{pH}$ dependent, any residual acid will change the $\mathrm{pH}$ of the $\alpha$-HIB solution and hence change the anion concentration, thereby altering the elution positions of the ions under study. A solution of $0.05 \mathrm{M} \alpha-\mathrm{HIB}$, adjusted to $\mathrm{pH}=4.47$ using concentrated $\mathrm{NH}_{4} \mathrm{OH}$, was then used to elute the activity from the column. Fractions were collected dropwise on $\mathrm{Pt}$ foils. The $\alpha-\mathrm{HIB}$ solution on the foil was evaporated on a hot plate and flamed to ensure that the activity would adhere to the foil and to remove any residual organic material. The samples were cooled, then placed on top of $300-\mathrm{mm}^{2} \mathrm{Si}-\mathrm{Au}$ surface barrier detectors and assayed for $\alpha$ and SF decay. The total time for this procedure was about six minutes from the end of collection until the first sample, and about nine minutes until the last sample was placed on the detectors. After the experiment, the foils were placed in front of an intrinsic Ge detector to determine the relative positions of the lanthanide tracers that were used in the experiment by assay of their $\gamma$-radiations. This was done to make certain that the column had performed as expected. At the end of each elution the column was stripped using 0.5 $\mathrm{M}, \mathrm{pH}=4.2 \alpha$-HIB to remove any remaining activity. The column was then prepared for the next experiment by rinsing it with $1 \mathrm{M} \mathrm{NH}_{4} \mathrm{Cl}$ and $\mathrm{H}_{2} \mathrm{O}$. Two columns were used so that the maximum number of experiments could be performed in the shortest time possible. A flow chart of this procedure is shown in figure 5 . 


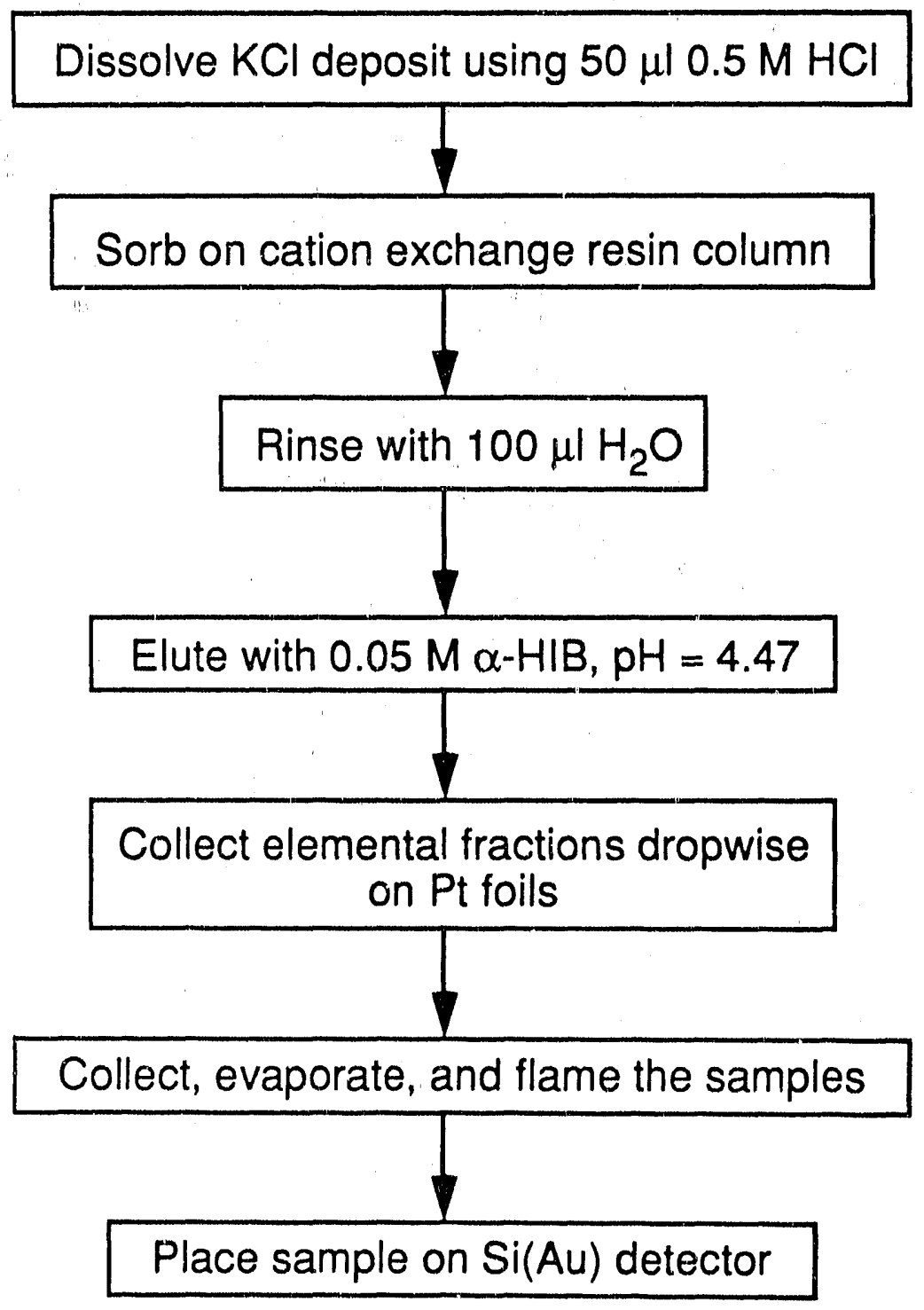

Figure 5: $\alpha-$ HIB chemistry flowchart 


\section{C2. ACCESS automated $\alpha$-HIB}

The construction of our computer controlled Automated Chromatographic Chemical Element Separator System, ACCESS [PAL88], allowed us to automate the $\alpha$-HIB chemical procedure. This has several immediate advantages over the manually performed separations. Primarily, the separation is much more reproducible than can be achieved manually. The separations can also be carried out faster, as more pressure can be applied from the solvent pumps than can be applied manually using gas pressure to the top of a resin column. ACCESS is an IBM-PC controlled column chromatography system that can be configured to do a variety of different chemical procedures. The system consists of off-the-shelf components including the PC, solvent pumps, a data acquisition board, several slider valves, and a fraction collector. The computer is an IBM-PC, into which a Data Translation DT-2801 data acquisition board is placed. The board translates the basica commands from the PC into signals appropriate for pump and valve control. Two LDC Cheminert Metering pumps from Milton Roy, along with a model AA Eldex metering pump are used to provide solvent flow through the system. The Eldex pump is connected to a six-way solvent selector valve to allow a total of eight separate solutions to be pumped through the system. Cheminert slider valves obtained from Rainin direct the flow of solvents through capillary tubes to the appropriate locations in the system. These are air actuated slider valves controlled by the PC. A Gilson model FC-203 fraction collector is used to collect the eluate from the column. The fraction collector is controlled through the PC's serial port. The analytical column can be run at ambient temperature or heated in specially constructed $\mathrm{Al}$ heater blocks. Resistive heaters plugged into a Variac control the temperature of the heating block. A sample injection loop is currently in use until an automated injection system can be made to collect the aerosols from the $\mathrm{He} / \mathrm{KCl}$ jet directly. Figure 6 shows the electronics of the ACCESS system, while figure 7 is a schematic of the chemistry side of the system. 
The procedure used for the ACCESS controlled $\alpha$-HIB experiments is as follows. Samples were collected on teflon squares at the aerosol jer collection site. The $\mathrm{KCl}$ aerosols were dissolved in $95 \mu \mathrm{l}$ of $0.05 \mathrm{M} \alpha-\mathrm{HIB}$, without $\mathrm{pH}$ adjustment. The sample was picked up in a plastic syringe and injected into the injection loop in ACCESS. The sample was then pumped from the injection loop into a $1.5-\mathrm{mm} \times 40-\mathrm{mm}$ glass column packed with Hamilton $12 \%$ crossinked, 7-10 $\mu \mathrm{m}$ particle size, cation exchange resin using the same $\alpha$-HIB solution that was used to pick up the sample. This $\alpha$-HIB solution will not elute the $3+$ actinide ions from the resin. The elution was carried out using 0.5 $\mathrm{M}, \mathrm{pH}=3.39 \alpha$-HIB solution, prepared by rdjusting the $\mathrm{pH}$ of a $0.5 \mathrm{M} \alpha$-HIB solution with $\mathrm{NH}_{4} \mathrm{OH}$. The samples were collected either on special miniature hot plates or in polypropylene tubes, depending on the needs of the experiment. The miniature hot plates were $2.5-\mathrm{cm} \mathrm{Al}$ cylinders into which a resistive heater plug was placed. The temperature was controlled using a Variac. Once the desired fractions were collected, the column was stripped using 1.0 M, pH $=4.2, \alpha$-HIB solution. The resin was then rinsed with $\mathrm{H}_{2} \mathrm{O}, 1 \mathrm{M}$ $\mathrm{NH}_{4} \mathrm{Cl}$, and then $\mathrm{H}_{2} \mathrm{O}$ again to prepare it for the next experiment. The samples were evaporated, flamed, and placed on Si-Au surface barrier detectors to assay for $\alpha$ and SF activities.

\section{C3. ACCESS Chemistry with Catcher Foils}

In some of the experiments that were performed on ACCESS the recoiling product atoms were caught in $1.6 \mathrm{mg} / \mathrm{cm}^{2}$ Au catcher foils. The catcher foils were removed from the target system following the end of the bombardment. The Au foil was then cut from the support ring and placed in a 15-ml centrifuge tube. Two drops of concentrated $\mathrm{FCl}$ and one drop of $\mathrm{HNO}_{3}$ were added to the tube, which was then heated in a hot water bath to speed the dissolution of the foil. The resulting solution was placed on the top of a 2$\mathrm{mm}$ diameter by $50-\mathrm{mm}$ long glass column packed with 200 mesh AG-1X8 anion 


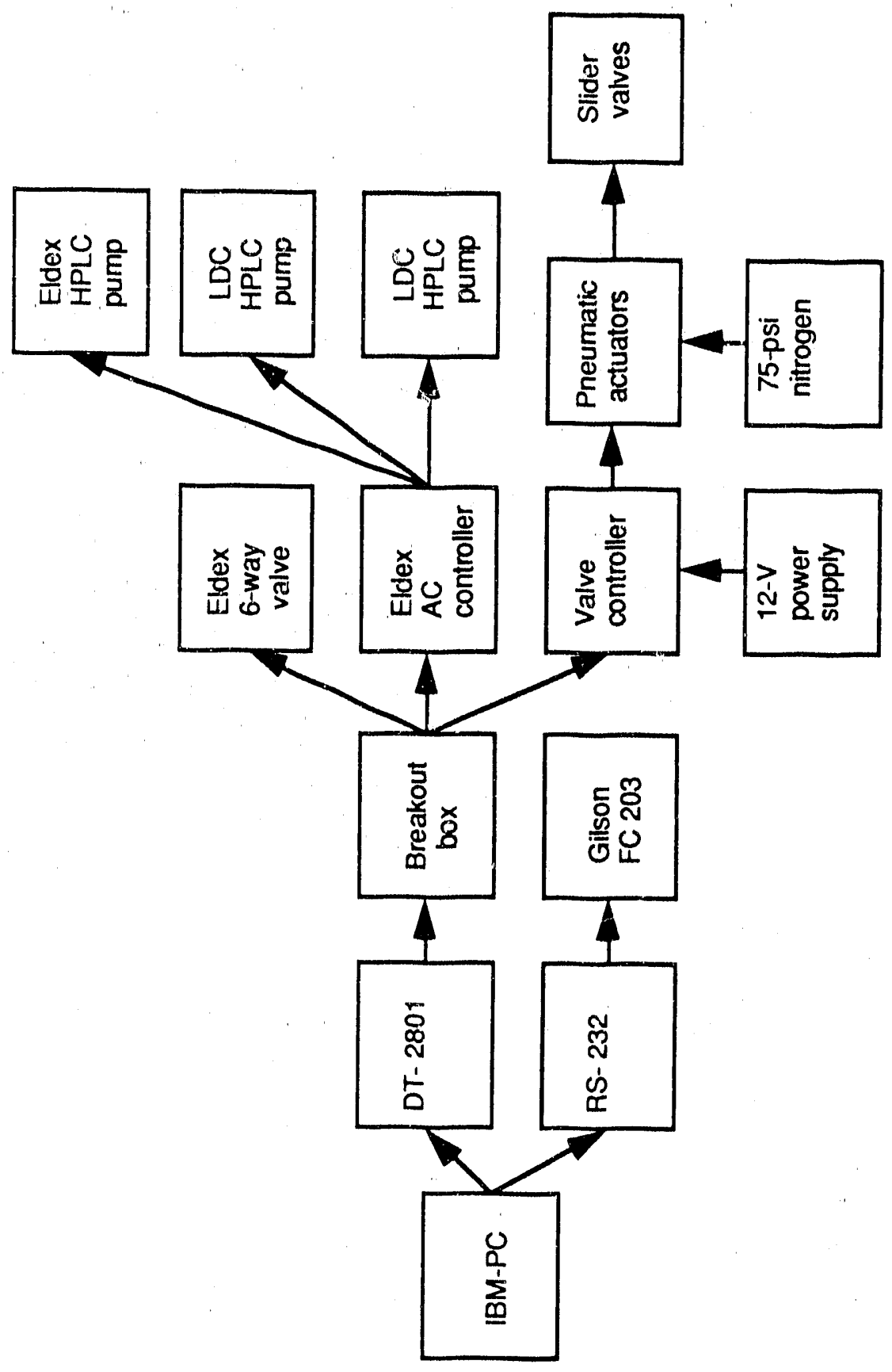

Figure 6: Electronics diagram of ACCESS 


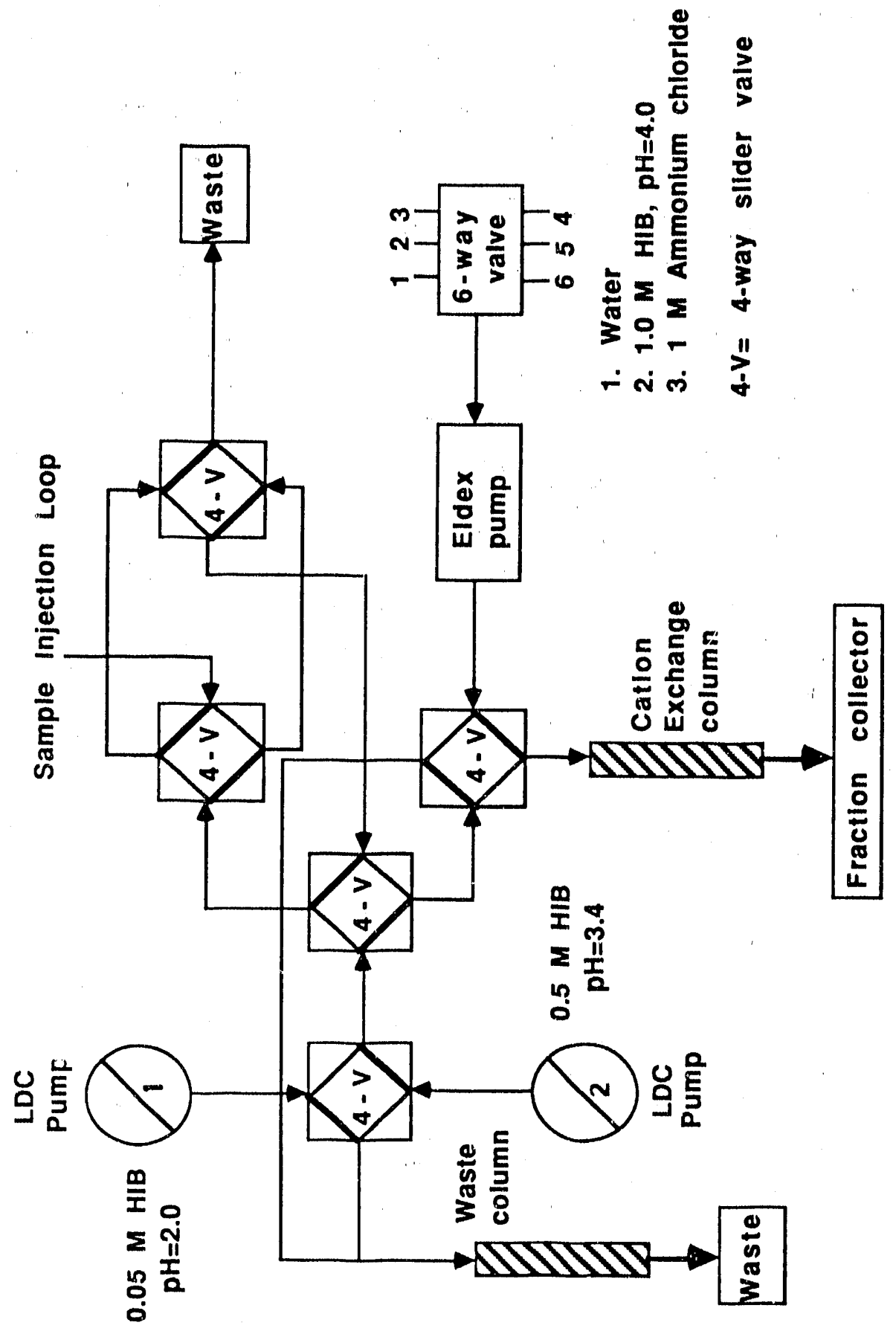

Figure 7: Flow diagram of the chemistry for ACCESS 
exchange resin from Bio-Rad Laboratories. The active solution was pushed onto the resin using $\mathrm{N}_{2}$ gas pressure. The actinide fraction was stripped from the column using concentrated $\mathrm{HCl}$. The eluate was collected in a glass planchette and evaporated beneath a heat lamp. After the concentrated $\mathrm{HCl}$ had evaporated, a small amount of $1 \mathrm{M} \mathrm{HCl}$ was added to the planchette and again taken to dryness. The sample was removed from the planchette with $95 \mu \mathrm{l}$ of $0.05 \mathrm{M} \alpha-\mathrm{HIB}, \mathrm{pH}=2.7$. A flow chart for this separation is shown in figure 8. The sample was then injected into ACCESS and the separation performed as in the gas jet collection type experiments. Individual elemental fractions were collected in polypropylene tubes. The samples were evaporated in many steps on $\mathrm{Ta}$ foils placed on top of washers on the top of a hot plate. The washers provided edge heating of the $\mathrm{Ta}$ foils, and helped retain the sample in the center of the foil during evaporation. Once dry, the samples were flamed to ensure good adhesion of the sample to the foil. They were cooled and placed on the Si-Au surface barrier detectors for radiation measurements.

\section{C3. Extractions}

Single step solvent extraction experiments provide one of the most rapid procedures that can be performed. When performed on a micro scale, the organic phase can be evaporated in a minimum time to give good, clean samples for radiation detection. In order to confirm the isotopic assignment of ${ }^{260} \mathrm{Lr}$ as an isotope of $\mathrm{Lr}$, solvent extraction experiments were performed that were the same as the TTA extractions that had been performed by Silva [SIL70] on 28-sec. ${ }^{256} \mathrm{Lr}$. Solutions of $0.1 \mathrm{M}$ TTA in MIBK were prepared by dissolving $2.22 \mathrm{~g}$ of TTA directly in $100 \mathrm{ml}$ of MIBK. Buffer solutions ranging in $\mathrm{pH}$ from 1.86 to 5.5 were prepared by adding $0.1 \mathrm{M}$ sodium acetate to either $0.1 \mathrm{M}$ acetic acid or $0.1 \mathrm{M}$ monochloroacetic acid solutions until the desired $\mathrm{pH}$ was attained. pH measurements were made lising a Beckman Futura II glass combination 


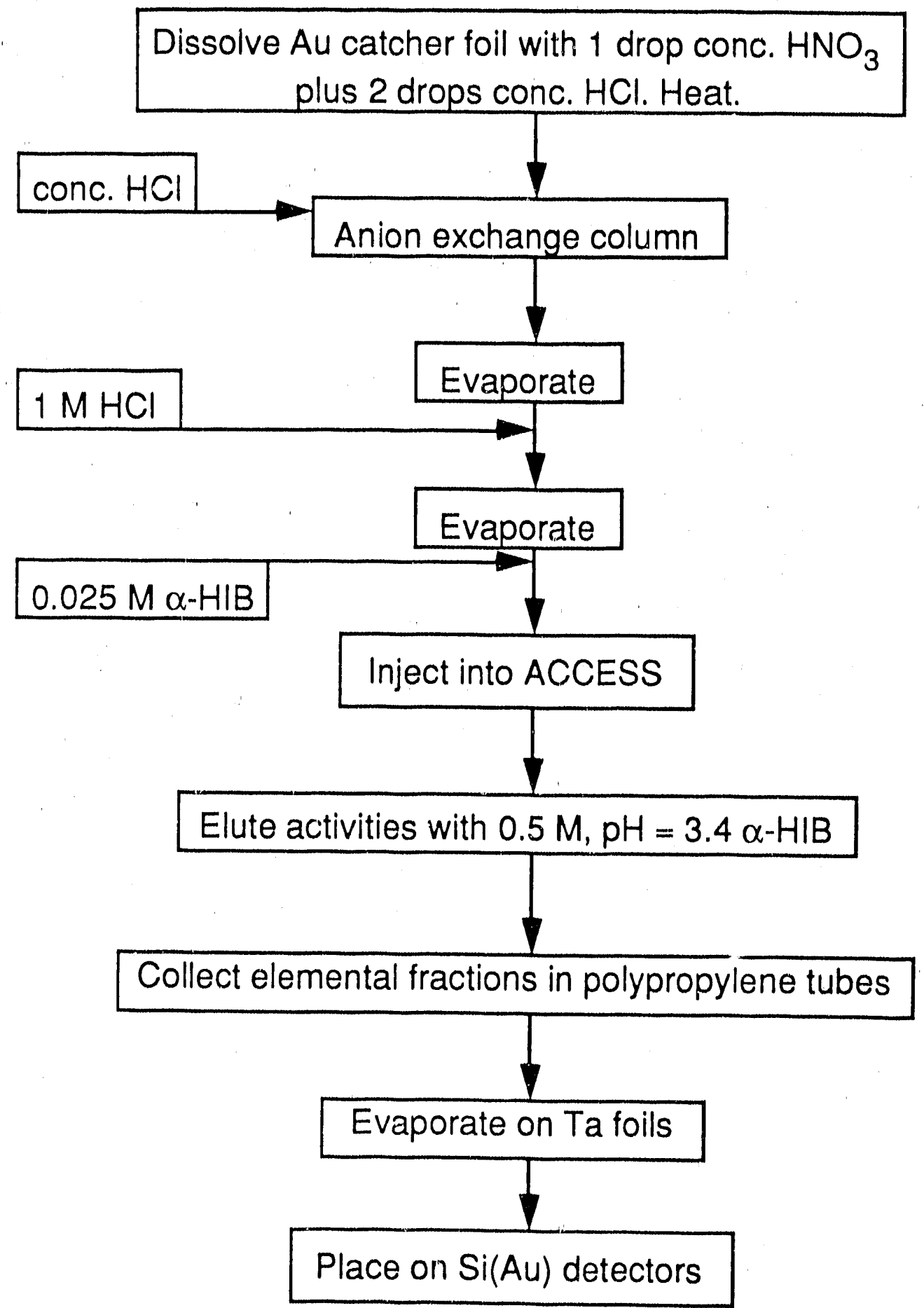

Figure 8: ACCESS $\alpha$-HIB procedure with Au catcher foils 
electrode and a Corning model 109 digital $\mathrm{pH}$ meter. Recoiling product atoms were transported via the $\mathrm{He} / \mathrm{KCl}$ aerosol jet to a collection site in a glove box placed on top of the experimental cave. The aerosols were dissolved with $50 \mu \mathrm{l}$ of the aqueous buffer solution, and placed in a $2-\mathrm{ml}$ centrifuge tube containing $50 \mu \mathrm{l}$ of the organic phase. The tube was heated in a hot water bath for 10 seconds, then mixed on an orbital shaker for 10 seconds. The tube was placed in a centrifuge and spun for about 15 seconds, long. enough for the centrifuge to attain its maximum speed. The tube was removed from the centrifuge, and the organic phase removed from the tube with a transfer pipette. The organic phase was placed on a $\mathrm{Pt}$ foil on top of a stack of washers on a hot plate and dried. The resulting sample was placed on a $\mathrm{Si}$-Au surface barrier detector and assayed for $\alpha$ and SF decay. This procedure took about 65 seconds to perform, and was repeated every 90 seconds.

Element 105, Ha, provides special experimental difficulties due to the fact that ${ }^{262} \mathrm{Ha}$, currently the longest-lived isotope of $\mathrm{Ha}$, has only a 34 second half-life. Methods for studying the chemical properties of $\mathrm{Ha}$ must, therefore, be done very rapidly, and must produce samples free of mass contaminants that degrade the resolution of alpha spectra. Ha must also be separated from the actinide elements, in particular Fm and Md. One such method is a single step solvent extraction separation. An industrial procedure [WER54] using methylisobutyl ketone, MIBK, was adapted for use on a micro-scale for studies of $\mathrm{Ha}$ chemistry. $\mathrm{Ta}$ and $\mathrm{Nb}$ can be separated from each other by selective extractions from strong mineral acid solutions into MIBK. MIBK is a low-boiling , b.p. $=114^{\circ} \mathrm{C}$, organic solvent that can be evaporated quite quickly, leaving no residue behind, and is thus well suited for fast chemical studies, and for producing good quality sources for alpha spectroscopy. Off-line tests using ${ }^{182} \mathrm{Ta}$ and ${ }^{249} \mathrm{Cf}$ showed that the extraction of Ta was quite good, while Cf did not extract at all. The procedure used was as follows. Aerosol particles from the $\mathrm{He} / \mathrm{KCl}$ jet were collected on a $\mathrm{Pt}$ foil. The $\mathrm{KCl}$ spot was dissolved using $20 \mu$ of a $3.8 \mathrm{M} \mathrm{HNO}_{3} / 1.1 \mathrm{M} \mathrm{HF}$ acid solution. The sample was removed from 
the $\mathrm{Pt}$ foil and placed in a $1 \mathrm{ml}$ centrifuge cone containing $20 \mu \mathrm{l}$ of MIBK. The solvents were mixed ultrasonically for about 2 seconds. A rnodified home humidifier was used to provide an ultrasonic mixing bath. Mixing was found to be complete in about 1 second, but to ensure that it was, a two-second mixing time was used in the experiments. The centrifuge tube was placed in a centrifuge that had been specially modified to achieve its maximum speed and come to a complete stop in about four seconds. The upper MIBK layer was transferred to a $\mathrm{Ni}$ foil that was being edge heated on a hot plate. After drying, the sample was flamed, cooled, and placed on Si-Au surface barrier detectors to measure the $\alpha$ and SF activity. The processing time for this procedure averaged about 65 seconds. This extraction procedure is displayed schematically in figure 9.

\section{C4. Adsorption procedure}

Group 4 and 5 elements are known to adhere quite well to glass surfaces. This fact was used to separate $\mathrm{Ha}$ from the actinide elements via a quick adsorption technique. The technique that was developed was found to produce excellent sources for alpha spectroscopy. This was also a separation that could be performed quite rapidly. The aerosols from the $\mathrm{He} / \mathrm{KCl}$ jet were collected on the substrate of choice for the experiment. In the case of the Ha experiments, a glass microscope cover slip was used. The subsuate was removed from the collection site and placed directly on a hot plate at its maximum temperature, $\cong 225^{\circ} \mathrm{C}$. Five $\mu$ l of a concentrated acid was placed on the KCl spot and fumed to dryness. For some of the test runs, this was repeated. After fuming, the substrate was rinsed with $1.5 \mathrm{M} \mathrm{HNO}_{3}$ to remove everything except the group 4 and 5 elements. The substrate was rinsed with acetone to help speed the drying of the substrate. It was placed in the stream of hor air from an electric heat gun for the final drying. The sample was placed either in front of an intrinsic Ge detector, or, as in the $\mathrm{Ha}$ experiments, on top of a Si-Au surface barrier detector. For the experiments on $\mathrm{Ha}$, two 


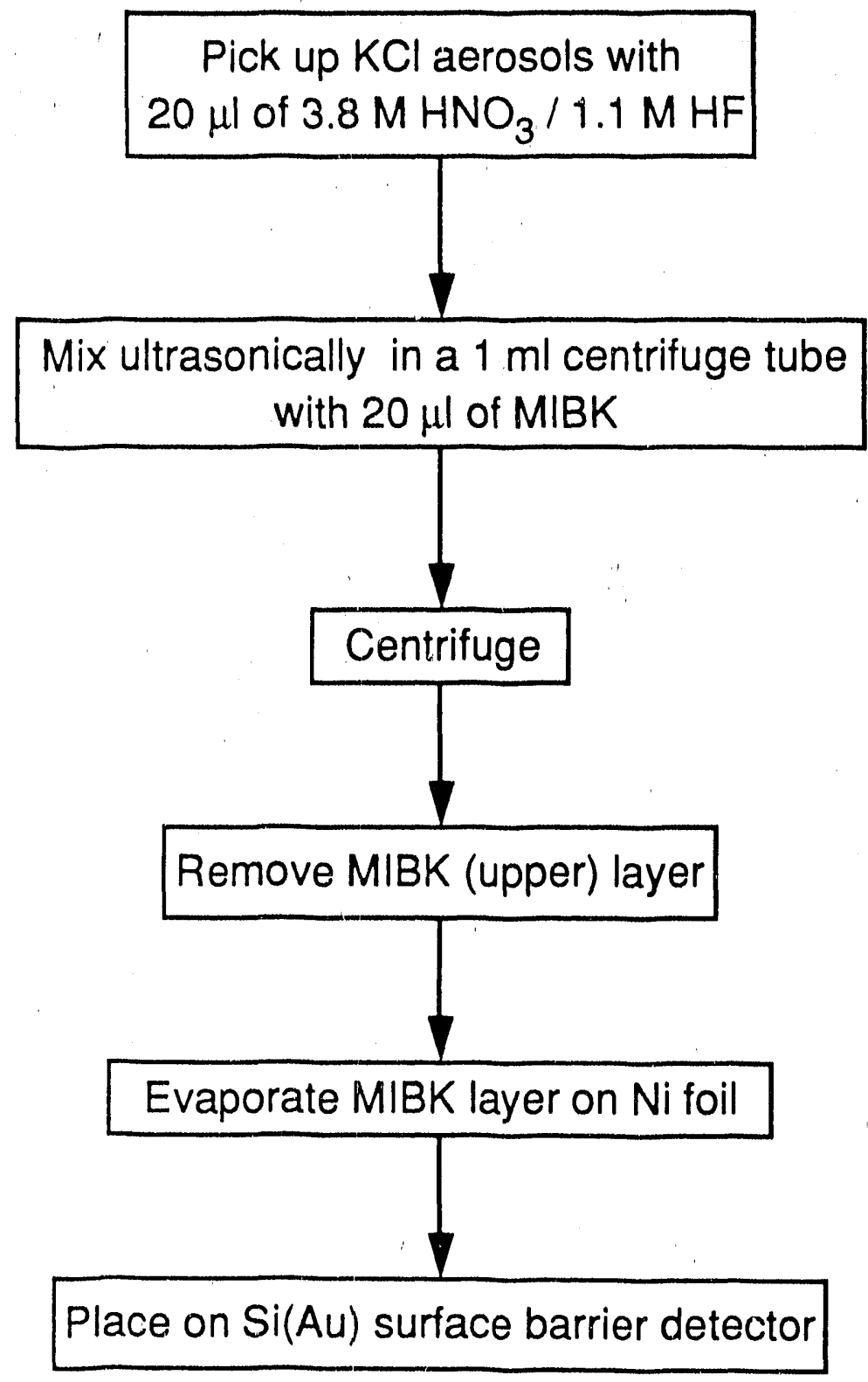

Figure 9: MIBK chemistry flowchart 
fumings with $\mathrm{HNO}_{3}$ were used, as this affords a crude separation between group four and group five elements, as observed in the test experiments. The procedure with two fumings took an average of 51 seconds to perform. The procedure is shown schematically in figure 10.

\section{Detector Systems}

Data collection at the cyclotron was performed using the Realtime data Acquisition and Graphics System, RAGS. RAGS is an LSI computer-controlled data acquisition system, shown schematically in figure 11. Alpha or spontaneous fission events from the sample on one of a series of ten detectors are recorded in one of the eight channels in one of four ORTEC AD811 analog-to-digital-converters (ADC). Fission events show up as overflow events in the appropriate alpha spectrum, and are mixed together in groups of five for the fission spectra. The detected event energies are stored digitally on magnetic tape. Each event has the time that it occurred stored with it, as well as the detector in which it was recorded. ORTEC $300 \mathrm{~mm}^{2} \mathrm{Si}(\mathrm{Au})$ surface barrier detectors were used.

The alpha spectrum was calibrated using a source of $212 \mathrm{~Pb}$, prepared by the recoil of daughter activities from a ${ }^{228} \mathrm{Th}$ source. ${ }^{212} \mathrm{Bi}$ and ${ }^{212} \mathrm{Po}$, the $\mathrm{B}^{-}$- decay daughters of ${ }^{212} \mathrm{~Pb}$, have alrha particles of $6.051 \mathrm{MeV}$ and $8.784 \mathrm{MeV}$, respectively. The fission spectrum was calibrated using a ${ }^{252} \mathrm{Cf}$ source.

Long-term counting was performed off-line in a separate laboratory. The signals from a series of eight ORTEC $100 \mathrm{~mm}^{2} \mathrm{Si}$-Au surface barrier detectors in separate vacuum chambers were fed into an ORTEC ADCAM data acquisition system. The ADCAM is controlled by an IBM-PC. Spectra are stored on the internal hard-rive of the PC.

Gamma counting, which was used primarily for off-line tracer tests of the procedures outlined above, was performed using intrinsic Ge detector spectrometer systems using Canberra's System 100 PC based data acquisition system. 


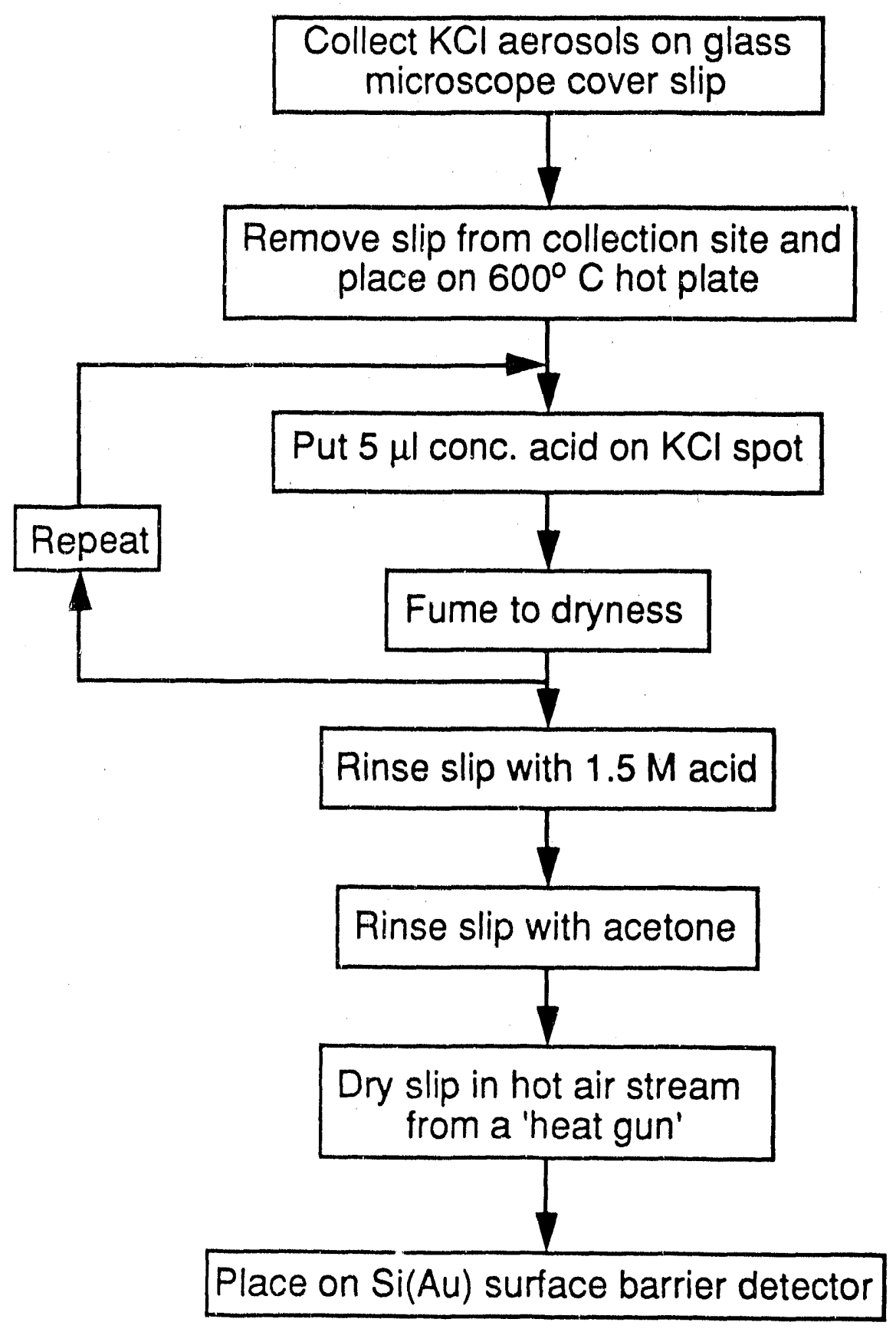

Figure 10: Adsorption chemistry flowchart 


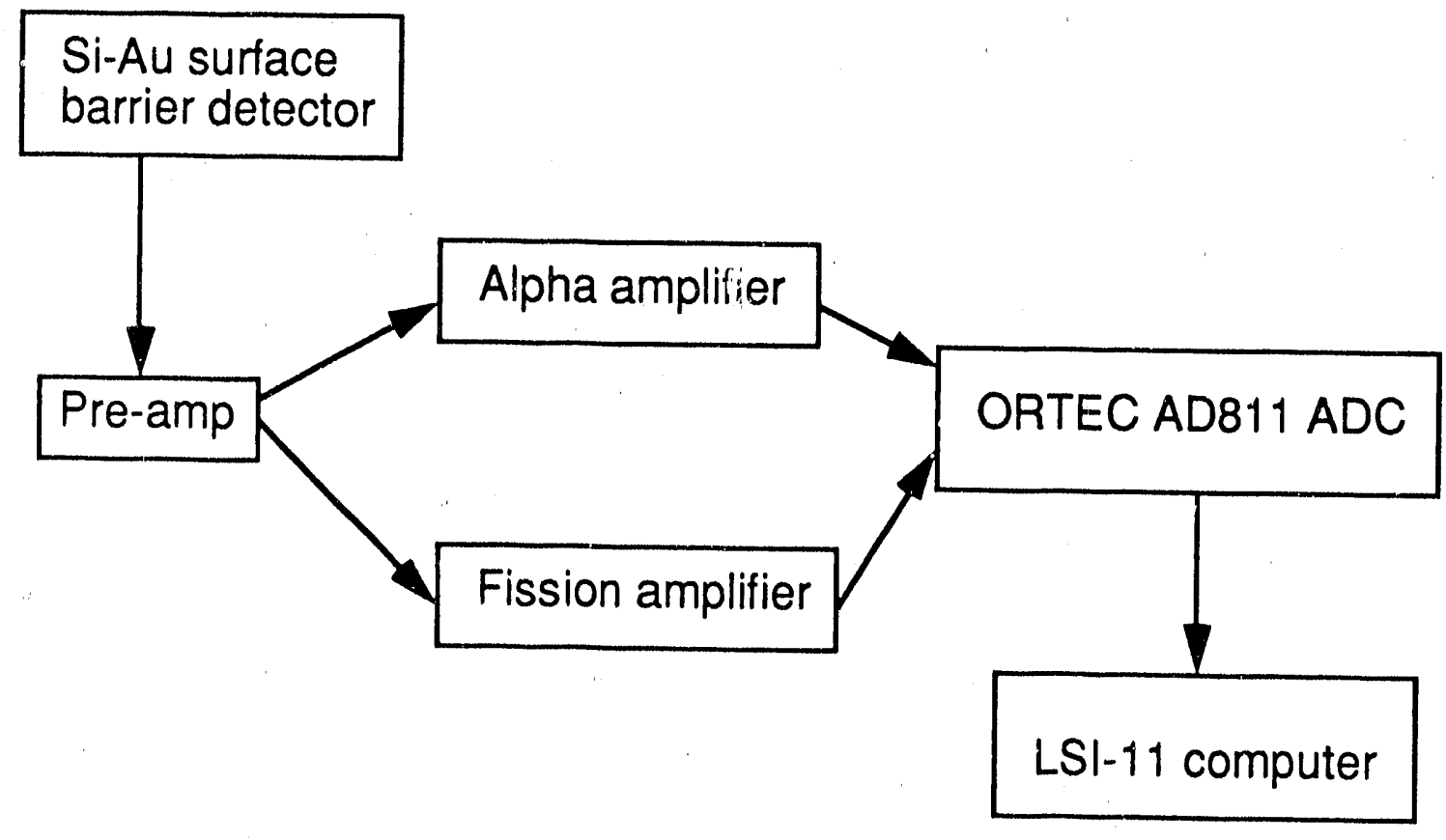

Figure 11: RAGS electronics schematic 


\section{E. Data Analysis}

The on-line data taken with the RAGS system were analyzed using a maximum likelihood computer code, utilizing a simplex method of decay curve fitting (MLDS) [GRE90]. The alpha and spontaneous fission spectra are integrated using a separate code especially written for the task. The data are first histogrammed into channel-by-channel spectra according to the time intervals that are set by the program user. The histogram files are then input into the integration program, which displays the individual spectra graphically on the computer screen. Regions of interest are determined, then written into separate decay-curve files. These files are then sent through the decay-curve fitting program and initial activities calculated for the components of the curve. The half-lives are allowed to vary freely in order to check how well the data match the known half-life values for the nuclides that are present in the sample.

Once initial activities are calculated for the time at the end of the bombardment; cross sections for the production of the nuclide can be calculated by standard means. The usual corrections for detector geometry, chemical efficiency, and time of separation are included in these calculations.

Some of the off-line data were analyzed using a least-squares curve fitting program. This method and the simplex method have been carefully compared and have been found to give results that are in good agreement [GRE90]. 


\section{Chapter III: Lawrencium Studies}

\section{A. Extraction Studies}

Since the discovery of a three-minute activity attributed to ${ }^{260} \mathrm{Lr}$ [ESK71], no chemical experiments have been performed to positively identify this activity as an isotope of Lr. All of the previous studies have been nuclear measurements only. The authors of these discovery experiments pointed out that the genetic link to $256 \mathrm{Md}$ that they found was far from conclusive, based upon the amount of background activity from ${ }^{256} \mathrm{Md}$ that had been produced directly. Further complications arise from the decay properties of 76 -minute ${ }^{256} \mathrm{Md}$, in that it has a $90.1 \%$ EC branch, which gives 2.63 -hour ${ }^{256} \mathrm{Fm}$, which has an SF branch of $93 \%$. The alpha particles emitted from ${ }^{256} \mathrm{Md}$ lie in the energy region masked by the alpha particles from ${ }^{254} \mathrm{Fm}$, which further obscures attempts to correlate the ${ }^{260} \mathrm{Lr}$ alpha decays with its daughter activity by nuclear spectroscopic means only. Additional chemical experiments were therefore necessary to positively identify this activity as an isotope of Lr.

A series of solvent extraction experiments was performed based upon the known extraction of $\mathrm{Lr}$ by TTA from various $\mathrm{pH}$ buffer solutions. These experiments were patterned after those performed by Silva et. al. [SIL70], who studied the extraction of 28 sec. ${ }^{256} \mathrm{Lr}$. Buffer solutions of acetic acid/sodium acetate and monochloroacetic acid/sodium acetate were prepared at $\mathrm{pH}=1.86,2.33,2.67,3.00,3.15,3.5$, and 4.0. The extraction behavior of ${ }^{260} \mathrm{Lr}$ was studied and compared to the extraction of isotopes of Fm, namely ${ }^{250} \mathrm{Fm},{ }^{254} \mathrm{Fm}$, and $252,255 \mathrm{Fm}$. More than one hundred separations were performed over the total set of extraction conditions. ${ }^{260} \mathrm{Lr}$ did not extract from the $\mathrm{pH}=$ 1.86 buffer solution. It began to extract, along with $\mathrm{Fm}$, from the $\mathrm{pH}=2.33$ buffer solution, and was essentially completely extracted from the $\mathrm{pH}=3.15$ buffer solution. The simplest method for examining the extraction data was to take the ratio of the ${ }^{260} \mathrm{Lr}$ 
activity to the ${ }^{254} \mathrm{Fm}$ activity. ${ }^{254} \mathrm{Fm}$ is produced by a transfer reaction on the ${ }^{249} \mathrm{Bk}$ target. ${ }^{254} \mathrm{Fm}$ is produced with a much higher yield than ${ }^{260} \mathrm{Lr}$, and has an alpha energy that is not obscured by other activities that are produced in this reaction. The ratio between these two activities, shown in table III, was found to be constant within experimental error. The extractions at $\mathrm{pH}=2.33$ gives values that are not consistent with the rest of the data, but the statistics are poor, and in fact, the value for $\mathrm{pH}=2.33$ is calculated upon observation of a single $8.03 \mathrm{MeV} \alpha$-particle. The errors on the ratios are $68 \%$ confidence limits taken from a Poisson distribution. Poor statistics were a result of the low extractability of the actinides and the consequently lower data rate for ${ }^{260} \mathrm{Lr}$. Many more extractions, on the order of 100 or so, would need to be performed to obtain good statistics at the lowest $\mathrm{pH}$ buffer solution extractions. A total of twenty-three 8.03 $\mathrm{MeV} \alpha$-particles were observed in these separations.

Table III: Extraction ratios

\begin{tabular}{|c|c|}
\hline $\mathrm{pH}$ & Ratio: $8.03 \mathrm{MeV} \alpha^{\prime} \mathrm{s} /{ }^{254} \mathrm{Fm} \alpha$ 's \\
\hline 2.33 & $0.0043_{-0.0043}^{+0.0056}$ \\
\hline 2.67 & $0.014_{-0.004}^{+0.006}$ \\
\hline 3.00 & $0.021_{-0.006}^{+0.009}$ \\
\hline 3.15 & $0.023_{-0.008}^{+0.013}$ \\
\hline 4.00 & $0.034_{-0.008}^{+0.012}$ \\
\hline
\end{tabular}

${ }^{255}$ No has decay properties that are nearly identical to ${ }^{260} \mathrm{Lr}$, so it is important to show that the activity produced is not due to this nuclide. The fact that the ratio between the Fm and Lr activity does not change shows that the nuclide being extracted is not ${ }^{255}$ No. The extraction of $\mathrm{No}^{2+}$ starts much later than the $3+$ actinides in this system. This 
observed behavior is consistent with the extraction properties that were measured using 25 -second ${ }^{256} \mathrm{Lr}$ [SIL70], confirming that the assignment of this isotope to an isotope of $\mathrm{Lr}$ is correct. The production cross section was also determined in these experiments. The data for the $\mathrm{pH}=4.0$ extractions were used for this determination. Based upon the observation of eight $260 \mathrm{Lr}$ decays, a detector geometry of $35 \%$, and an overall experimental yield of $11 \%$, the cross section was found to be $8.3 \pm 1.7 \mathrm{nb}$. The error limit is statistical plus an additional ten percent to account for variations in the beam intensity and gas jet yields that are otherwise not accounted for. The cross section determination makes it possible to determine the feasibility of further chemical experimentation. This determination is important to show that an experiment can be performed in a reasonable amount of cyclotron beam time. This determination showed that it is possible to perform further chemical experiments using ${ }^{260} \mathrm{Lr}$ produced in this manner.

\section{B. Ion Exchange Studies}

The second set of chemical studies on $\mathrm{Lr}$ was also performed using the isotope ${ }^{260} \mathrm{Lr}$. The three minute half-life of this isotope requires that the chemical procedure be done rapidly, but it does allow time for elution from a cation-exchange resin column using $\alpha$ HIB. The procedure must also remove any contaminants that can be confused with ${ }^{260} \mathrm{Lr}$, in particular ${ }^{255} \mathrm{No}$, which has an almost identical half-life and nearly the same alphaparticle energies. The standard reduction potential for the $\mathrm{No}^{3+} / \mathrm{No}^{2+}$ couple has been determined [SIL69] to be $+1.4 \pm 0.2 \mathrm{~V}$. Only under strongly oxidizing conditions will nobelium exist as $\mathrm{No}^{3+}$. The fact that No exists as a $2+$ cation in aqueous solution means that it will not elute near $\mathrm{Lr}$ in the $\alpha$-HIB system. In fact, $2+$ species will elute much later than $3+$ cations with this technique. This is shown in figure 12 , where the relative elution positions of $\mathrm{Eu}^{3+}, \mathrm{Ba}^{2+}$, and $\mathrm{Ra}^{2+}$ are compared. Note also that $\mathrm{Lr}$ 


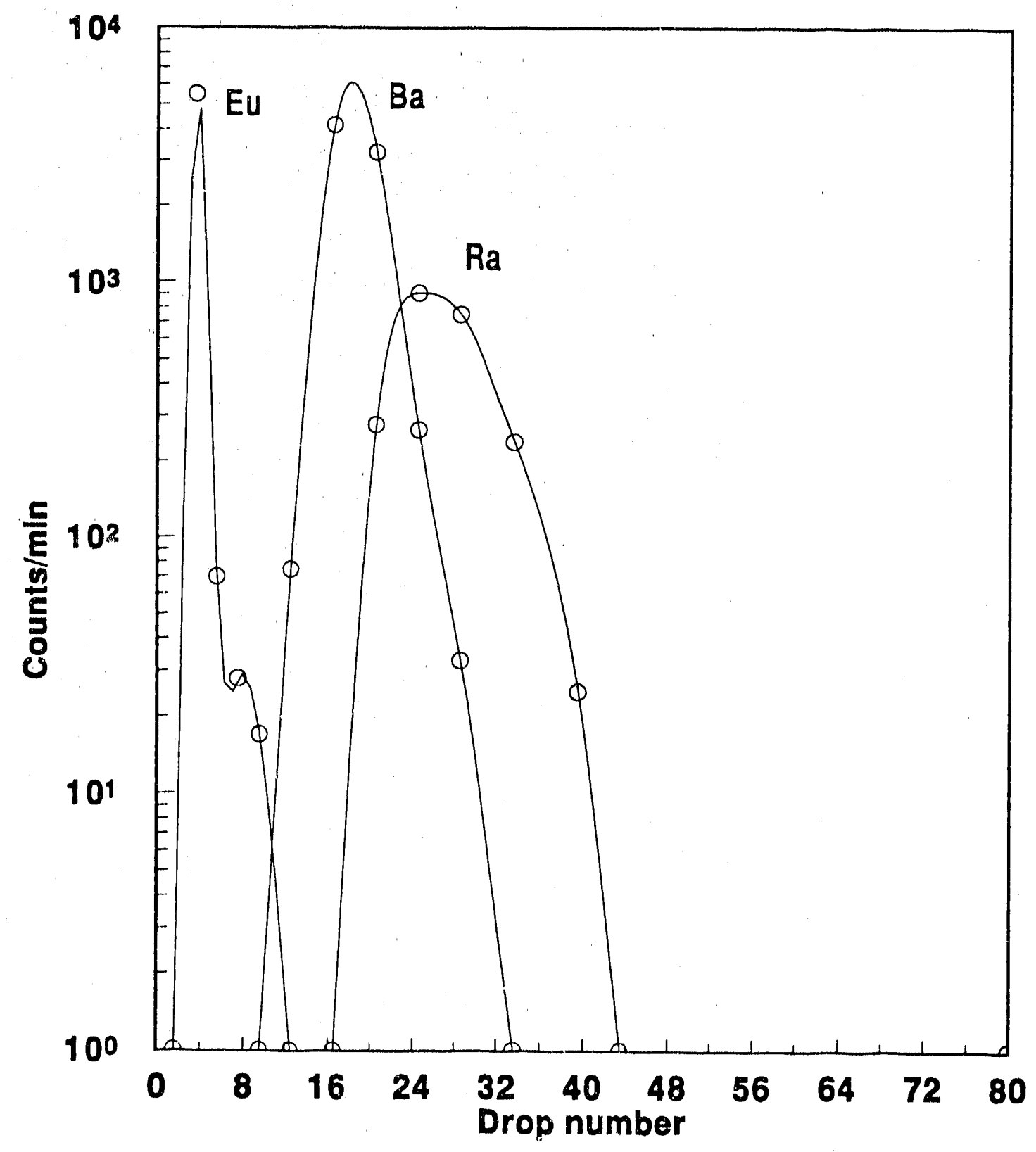

Figure 12: Elution of $\mathrm{Eu}^{3+}, \mathrm{Ba}^{2+}$, and $\mathrm{Ra}^{2+}$ from cation resin with $\alpha$-HIB 
will elute with $\alpha$-HIB much sooner than $\mathrm{Eu}$, so the separation from $\mathrm{No}^{2+}$ will be complete. Counting samples must also be as clean as possible, so that the best alpha spectrum may be obtained. Again, this requirement is met in the $\alpha$-HIB elution system. Sample sizes from manually operated $\alpha$-HIB separations are usually on the order of two drops, which can be evaporated and flamed to give very clean sources for alpha counting. The procedure can be completed in a short period of time, so that an excess of cyclotron time is not necessary to produce a meaningful result.

The determination of the ionic radius and thus the hydration enthalpy is facilitated, as was mentioned earlier, by the linear relationship between the crystal ionic radius and elution position for cations from a cation-exchange resin column. The species being compared must be in the same charge state as well as the same chemical form. The lanthanide and heavy actinide elements have been shown to exist in the same chemical form in aqueous solutions[CHO61]. Both sets of ions show the predominant $3+$ oxidation state in solution except for $\mathrm{No}^{2+}$. Thus, comparing the actinide ions of interest with heavy lanthanide tracers of known ionic radius is a valid method for determining the ionic radius of $\mathrm{Lr}^{3+}$.

A system in common use for the separation of the heavy $3+$ actinides and the lanthanide series is the elution of these ions from a cation-exchange resin column with $\alpha$ HIB. The $\alpha$-HIB separation depends on the ionic radius, with smaller ions being eluted from the culumn first. In the lanthanide and actinide series these are the highest $\mathrm{Z}$ ions, since the ionic radius contracts with increasing $\mathrm{Z}$. There is still some doubt as to the correct mechanism for this separation, but the most plausible explanation is that the $\alpha$ HIB anion forms the strongest complexes with the smallest ions. This results in the smallest ions being moved down the resin bed faster; hence, the highe $\mathrm{z} Z$ element will be the first to come off the column. It has been shown by Choppin [CHO61] that the $\alpha$ HIB complex that is formed at the $\mathrm{pHs}$ and concentrations of these studies involves four $\alpha$-HIB anions, and thus a $1^{-}$anionic species is being eluted from the column. Figure 13 
displays the linear relationship between the $\log$ of the distribution coefficient and the crystal ionic radius for the heavy lanthanide ions and for the heaviest actinide ions. The lanthanide points are plotted using relative elution positions, which are directly related to the distribution coefficients, from Smith and Hoffman [SMI56] and the ionic radius values from Templeton and Dauben [TEM54]. The values of the $\mathrm{Cf}^{3+}$ and $\mathrm{Es}^{3+}$ ionic radii are taken from Haire and Baybarz [HAI73]. The figure shows the known elution positions for the $\mathrm{Md}^{3+}$ and $\mathrm{Fm}^{3+}$ ions. $\mathrm{Md}^{3+}$ and $\mathrm{Fm}^{3+}$ do not have experimentally measured ionic radii, and the positions on the plot represent their elution positions relative to the lanthanide elements.

By examining the last few lanthanide and actinide elements, it is possible to estimate where $\mathrm{Lr}^{3+}$ would be expected to elute. Keeping in mind that nobelium is between $\mathrm{Md}$ and Lr, it would be expected that $\mathrm{Lr}$ should elute near the Tm position. The ideal lanthanide tracers to use for this are $\mathrm{Yb}$ and Er, leaving out $\mathrm{Tm}$, as it is known that any mass of an element in the system can affect the observed elution positions of the element and subsequent elements in the separation system.

Several calibration runs on the manually operated column were performed to check the elution positions and the reproducibility of the separation. The results of these tests are shown in figure 14. Note in particular the excellent separation between the $\mathrm{Yb}$ and $\mathrm{Er}$ tracers. The column separation was also found to be quite reproducible, as subsequent tests showed no change in the elution positions larger than one drop. Also note how soon the $\mathrm{Yb}$ is being eluted from the resin column. This indicates that the elution can be carried out in a minimum amount of time and still give a good separation between adjacent elements. The separation between these two elements could be increased by simply lowering the $\alpha$-HIB concentration; however, this would result in a much longer elution time.

The first set of on-line experiments was performed with the $\mathrm{Yb}$ and Er tracers present to make sure that the column behaved as it had in the calibration runs, and to give 


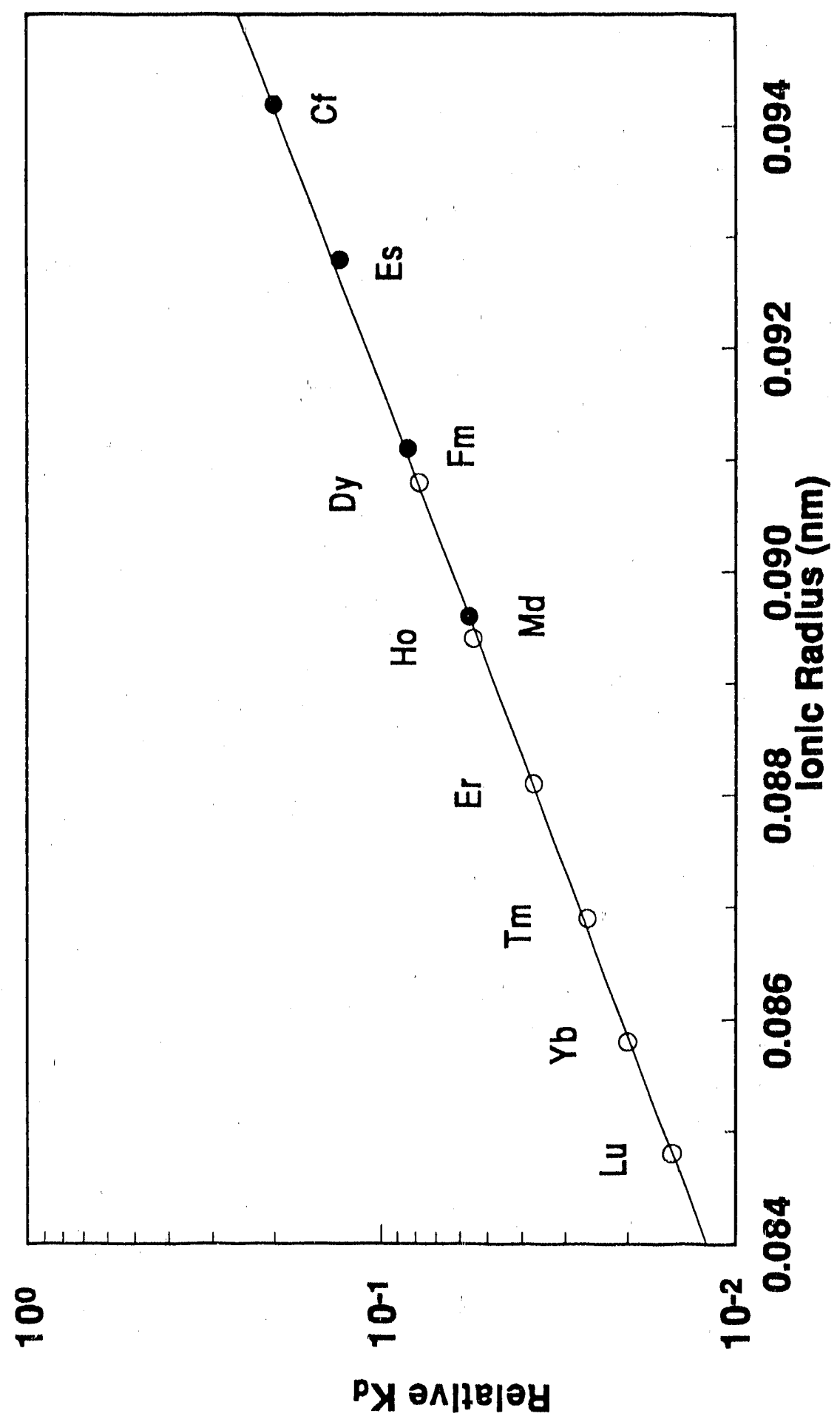

Figure 13: Ionic radius vs. Relative $\mathrm{K}_{\mathrm{d}}$ for heavy lanthanide and actinide $3+$ ions for elutions from cation resin with $\alpha$-HIB. Relative $\mathrm{K}_{\mathrm{d}}=\mathrm{K}_{\mathrm{d}} /\left(\mathrm{K}_{\mathrm{d}}\right)_{\mathrm{Cm}}$. 


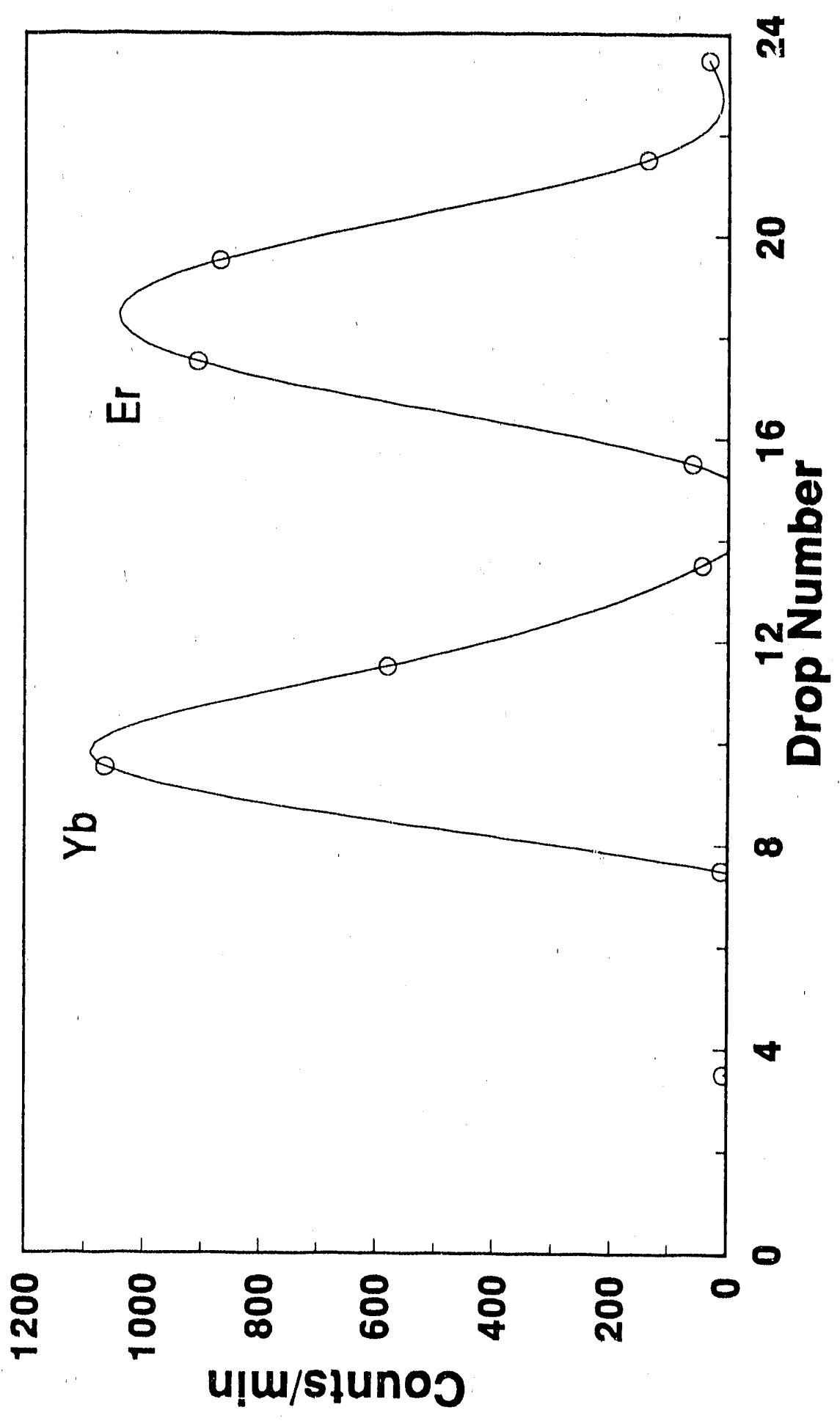

Figure 14: Elution curve for $\mathrm{Yb}^{3+}$ and $\mathrm{Er}^{3+}$ from the Dionex cation resin column with $\alpha$-HIB 
markers in the elution so that all elutions could be compared on the same basis. A total of 48 experiments was performed using the $\mathrm{Yb}$ and Er tracer activities to mark the elution position of Lr. No $\alpha$ events attributable to $260 \mathrm{Lr}$ were found near the Tm elution position. In two of the experiments, $\alpha$ events from ${ }^{260} \mathrm{Lr}$ were found very close to where the Er tracer was coming off the column. This result was somewhat unexpected, so it was necessary to alter the process to look near the Er elution position.

The second set of elution experiments used Tm and Ho tracers to mark the elution positions from the column. The eluting solution was kept the same, which meant that the activities were coming off the column a little later than in the elution with the $\mathrm{Yb}$ and $\mathrm{Er}$ tracers. A total of 29 experiments was performed with the Tm and Ho tracers, with a total of seven ${ }^{260} \mathrm{Lr}$ atoms being detected near the Er elution position. The alpha-particle energies were consistent with the known alpha-decay energy of $8.03 \mathrm{MeV}$. The most probable half-life of $2.7_{-0.9}^{+1.7}$ minutes was measured, and is consistent with the known half-life of $3.0 \pm 0.5 \mathrm{~min}$ [ESK 71] for this isotope. A possible contamination of the samples from ${ }^{223}$ Ac could give $\alpha$ events that would appear to be ${ }^{260} \mathrm{Lr}$ events. The decay chain of ${ }^{223} \mathrm{Ac}$ is shown in figure 15. The data were checked for correlations between the $8.03 \mathrm{MeV}$ alpha events and the two parent isotopes for this chain. No correlations were found, indicating that ${ }^{260} \mathrm{Lr}$ was definitely the isotope that was present in the samples. Additionally, Ac should elute much later than Lr, so to get Ac contamination it is necessary for this activity to build up on the column and run off in later elutions. Given the short half-life of ${ }^{223} \mathrm{Ac}$, this is a rather unlikely occurrence. With these facts, it is possible to conclude that $\mathrm{Lr}$ was present in these experiments, and was found to elute very near the elution position of Er. The result of these experiments is plotted in figure 16. From this plot, the ionic radius of the $\mathrm{Lr}^{3+}$ aqueous ion can be inferred. A value of $0.0886 \pm 0.0003 \mathrm{~nm}$ is derived from the relative elution positions. The error limits on the radius value represent the uncertainty in the elution positions as measured from separate 


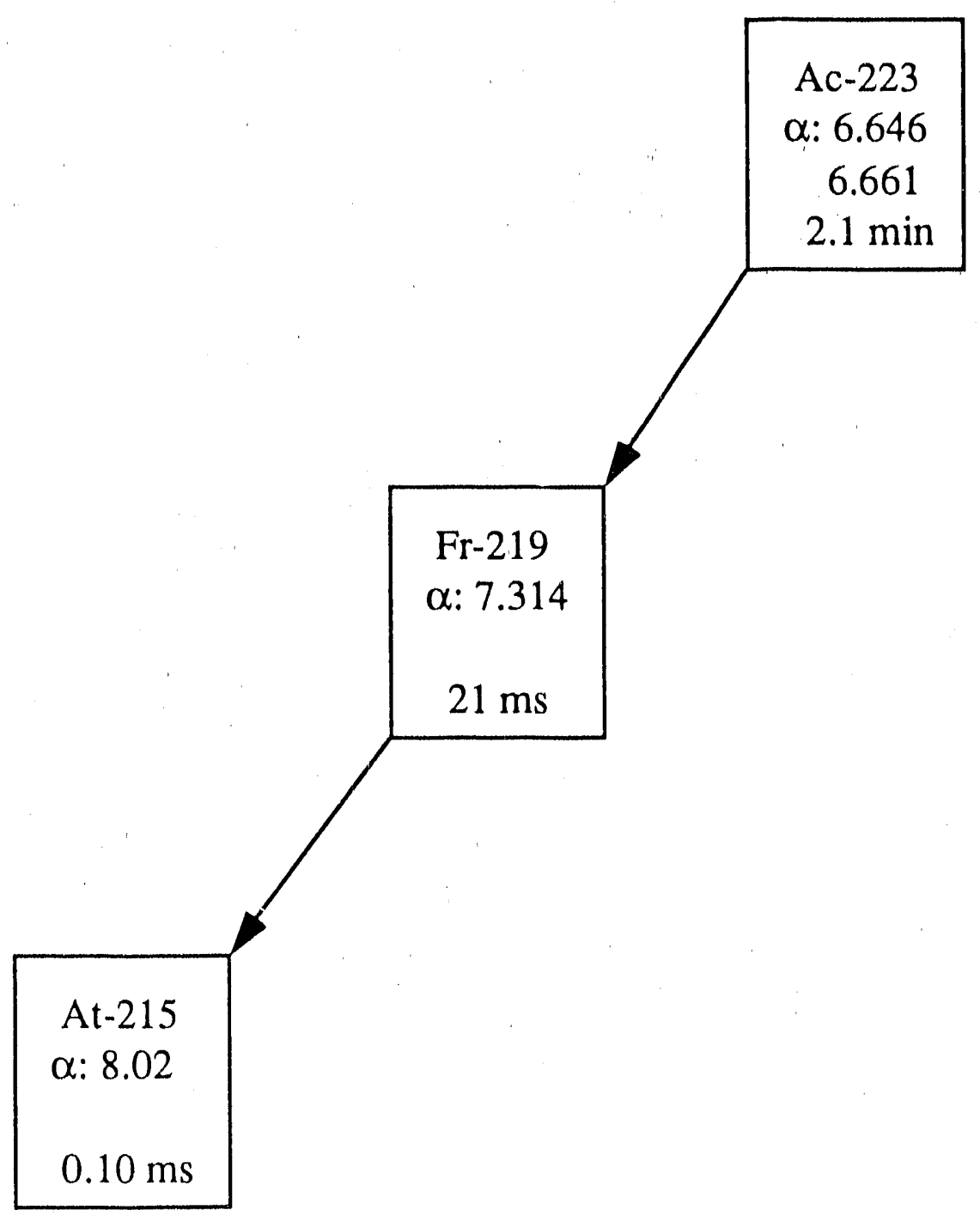

Figure $15:{ }^{223} \mathrm{Ac}$ decay chain 


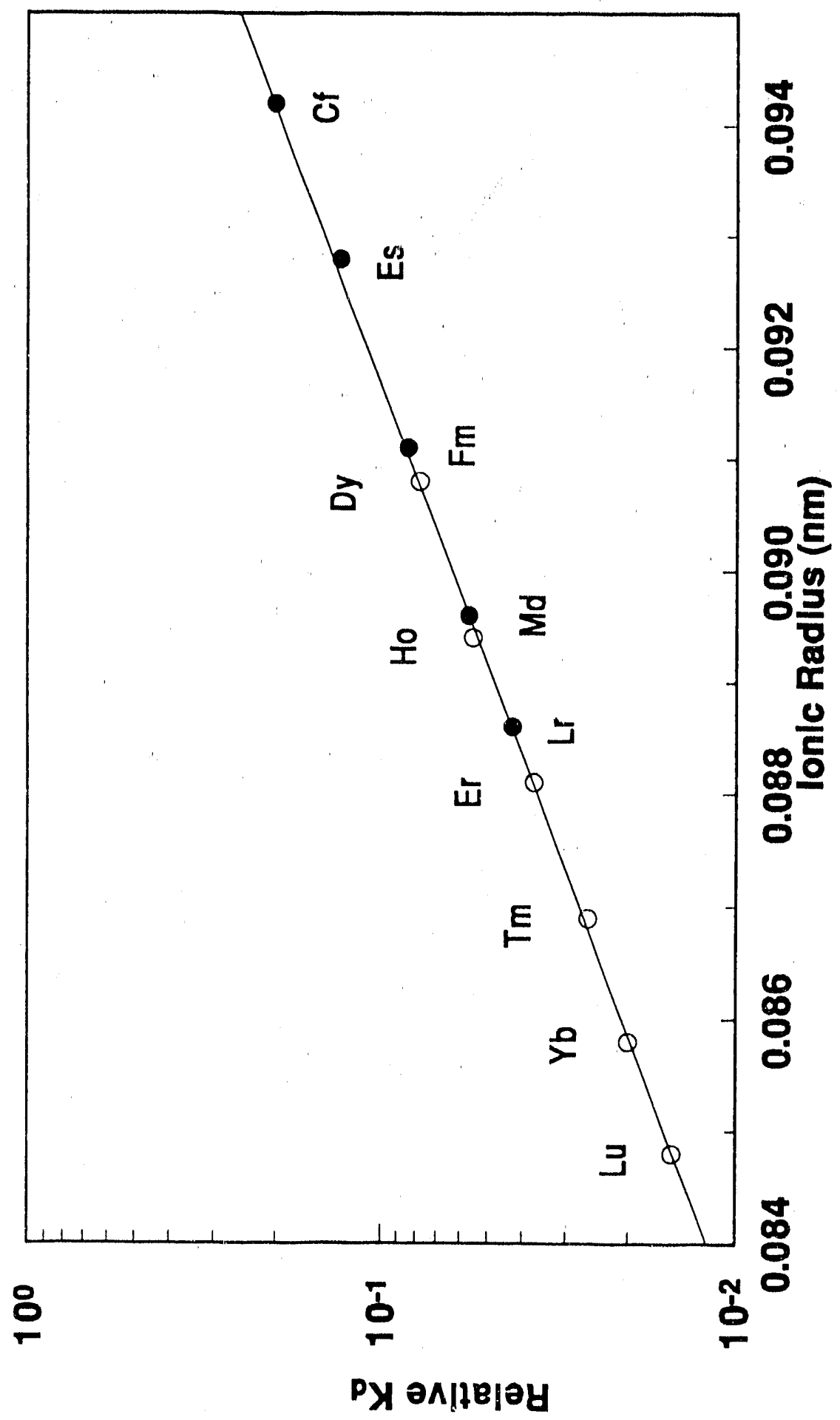

Figure 16: Ionic radius vs. Relative $\mathrm{K}_{\mathrm{d}}$ showing $\mathrm{Lr}$ position 
experiments, and the distribution of the observed $\mathrm{Lr}$ daıa points. This value, when inserted in the empirical Born equation, eq. 1, p. 7, gives a calculated enthalpy of hydration of $-3622 \pm 60 \mathrm{~kJ} / \mathrm{mol}$.

Subsequent to this work the elution position has been determined using an automated system by a multi-national collaboration [BRU88] and found to be $0.0881 \pm 0.00(1 \mathrm{~nm}$, in agreement with the initial result but with better statistics. The elution positions of $\mathrm{Md}^{3+}$ and $\mathrm{Lr}^{3+}$ were determined simultaneously by this collaboration. The hydration enthalpy was found to be $-3689 \pm 13 \mathrm{~kJ} / \mathrm{mol}$, again in agreement with the initial result.

\section{Production of ${ }^{261} \mathrm{Lr}$}

The production of ${ }^{261} \mathrm{Lr}$ by interactions of ${ }^{18} \mathrm{O}$ with ${ }^{248} \mathrm{Cm}$ can take place by one of two methods. The first is by electron capture in ${ }^{261} \mathrm{Rf}$ produced in the ${ }^{248} \mathrm{Cm}\left({ }^{18} \mathrm{O}, 5 \mathrm{n}\right){ }^{261} \mathrm{Rf}$ reaction. No electron-capture branch in ${ }^{261} \mathrm{Rf}$ has yet been experimentally observed. Using $\mathrm{Q}_{\mathrm{EC}}=2.7 \mathrm{MeV}$, assuming only a parity change for the beta decay, and a loeft $=6$, a partial EC half-life of 13.3 minutes results. This gives an EC branch of about $8 \%$. The second method of production is directly via the ${ }^{248} \mathrm{Cm}\left({ }^{18} \mathrm{O}, \mathrm{p} 4 \mathrm{n}\right){ }^{261} \mathrm{Lr}$ reaction. This type of compound nucleus pxn out reaction is not expected to have a large cross section in the heavy element region. The production of ${ }^{261} \mathrm{Rf}$ via the compound nucleus reaction is known [GHI70A] to have a cross section of 5 $\mathrm{nb}$ for an incident $18 \mathrm{O}$ energy of $97 \mathrm{MeV}$.

${ }^{261} \mathrm{Rf}$ is known to be an alpha-emitting isotope with a $65 \pm 10 \mathrm{sec}$. half-life [GHI70A]. An experiment to look for an electron-capture-decay branch in this isotope was performed. The $\mathrm{He} / \mathrm{KCl}$ aerosol jet was used to collect the reaction products. The collection can take place for a long time, as the ${ }^{261} \mathrm{Rf}$ will electron capture at the

collection site to 39 minute ${ }^{261} \mathrm{Lr}$. A series of experiments was performed with a collection time of 40 minutes. The aerosols were dissolved and injected into ACCESS, 
which then performed an $\alpha$-HIB separation based upon the previously determined elution position. Clean separation of the $\mathrm{Lr}$ fraction from the Md fraction is absolutely necessary, since ${ }^{261} \mathrm{Lr}$ has been observed to decay by fission [HOF87]. ${ }^{256} \mathrm{Md}$, which is a 75 minute e ectron-capturing isotope, will decay to 2.63 -hour $256 \mathrm{Fm}$, which has a $91.9 \%$ SF decay branch. It is therefore highly desirable to elute the Md fraction as far away from the $\mathrm{Lr}$ fraction as is possible. The $\mathrm{Lr}$ fraction was eluted with $0.5 \mathrm{M} \alpha-\mathrm{HIB}, \mathrm{pH}=3.37$. The $\mathrm{Lr}$ fraction came 80 drops, 6.2 free column volumes (FCV), FCV $=13$ drops, after the start of the elution, and a fifty drop (3.8 FCV) fraction was taken. The Md fraction started about 15 drops (1 FCV) later, so there was a very good separation between the two elements. Due to the sample size, the Lr fraction was collected in a polypropylene tube and evaporated in several portions on a Pt foil. The foil had a 'dimple' in the center of it and was placed on a washer on the top of a hot plate. This method has been found to keep the sample in the center of the foil better than any other method. After complete evaporation, the sample was flamed and placed on a Si-Au surface barrier detector. The processing time for this experiment was about 18 minutes from the end of collection until the sample was placed on the detector.

The decay curve of the observed fission events, which was fit with the MLDS [GRE90] program, is shown in figure 17. The half-life obtained from the MLDS fit is $44_{-11}^{+17}$ minutes, which agrees quite well with the value of $39 \pm 12$ minutes obtained in the discovery experiments [LOU87]. This is, despite the larger error-limits, a much better value for the half-life of this nuclide, as the discovery experiment had several disadvantages that were not present in this experiment. First of all, in the discovery experiment, ${ }^{261} \mathrm{Lr}$ was produced via transfer reactions from interactions of a ${ }^{22} \mathrm{Ne}$ beam with an ${ }^{254}$ Es target. This produces a large amount of ${ }^{262} \mathrm{~L}$.r as well as ${ }^{261} \mathrm{Lr}$. In the current experiment, as can be seen from the decay curve, there is almost no contribution from a long-lived component. The chemical procedure used in the discovery experiment 


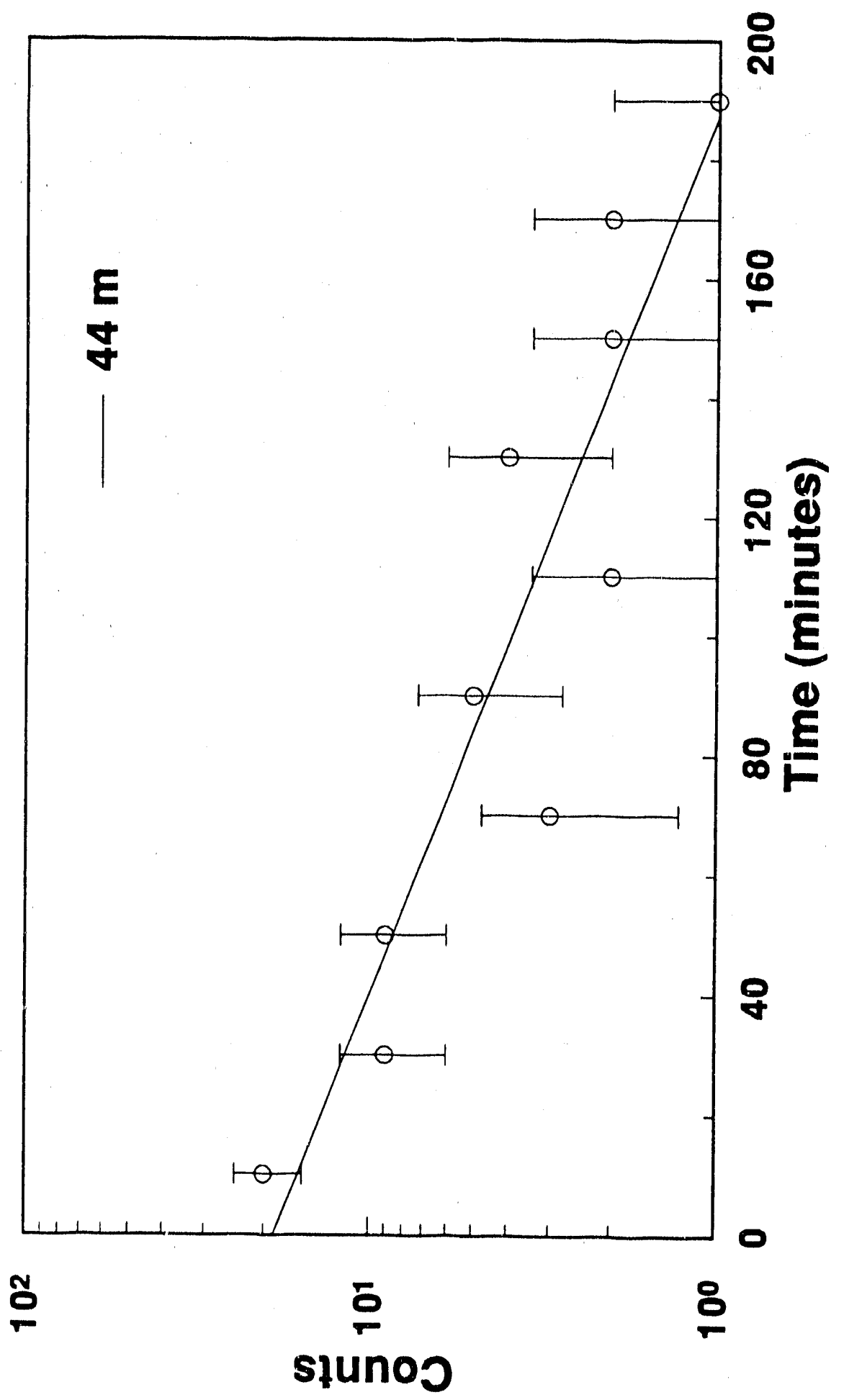

Figure 17: Decay curve for the ${ }^{261} \mathrm{Lr}$ fission events 
for the separation of a $\mathrm{Lr}$ fraction took approximately two hours, which is two and a half half-lives of the ${ }^{261} \mathrm{Lr}$. The chemical processing time in the current experiment averaged about seventeen minutes from the end of collection until the sample was placed on the detectors. The long processing time in the discovery experiments as well as the amount of ${ }^{262} \mathrm{Lr}$ present make a very accurate determination of the half-life of ${ }^{261} \mathrm{Lr}$ difficult. Also, very little can be determined about the fission properties of this isotope, as there is no way of positively knowing in the early part of the data collection which nuclide fissioning.

Figure 18 shows an energy histogram of the 62 fission events that were observed. Also shown in this plot is a line for the energy calibration using a ${ }^{252} \mathrm{Cf}$ source. From the appearance of this spectrum some preliminary conclusions about the fission properties of the fissioning species can be made. It is not yet clear whether ${ }^{261} \mathrm{Lr}$ will fission from the ground state or following electron capture to ${ }^{261}$ No, as has been observed for ${ }^{262} \mathrm{Lr}$ [LOU87]. Whatever the fissioning species is, it has reasonably symmetric mass division with a total kinetic energy of about $210 \mathrm{MeV}$, which is calculated by taking a weighted average over the ${ }^{252} \mathrm{Cf}$ spectrum, normalizing to $\mathrm{TKE}=186.5 \mathrm{MeV}$ for ${ }^{252} \mathrm{Cf}$ [HOF87A], and scaling the ${ }^{261} \mathrm{Lr}$ data to it. This TKE is not unusual for other fissioning isotopes in the region, which can be seen by plotting the TKE vs. $Z^{2} / A^{1 / 3}$ [HOF89] as shown in figure 19. Shown in the figure are the fits from the Viola [VIO66] and Unik et. al. [UNI74] systematics. Since only one fission fragment was collected, full information about the mass division and total kinetic energy are not available, thus the data point for ${ }^{261} \mathrm{Lr}$ is shown as a hollow square. The value for the TKE is estimated to be no better than $\pm 10 \mathrm{MeV}$.

The experimental production cross section for ${ }^{261} \mathrm{Lr}$ from this reaction was calculated to be $700 \pm 70 \mathrm{pb}$ based on the detected SF's. This would correspond to an EC branch in ${ }^{261} \mathrm{Rf}$ of $14 \pm 1.4 \%$. This is about a factor of two above the $8 \%$ estimate, which was calculated at the beginning of this section; however, it is not out of line with this 


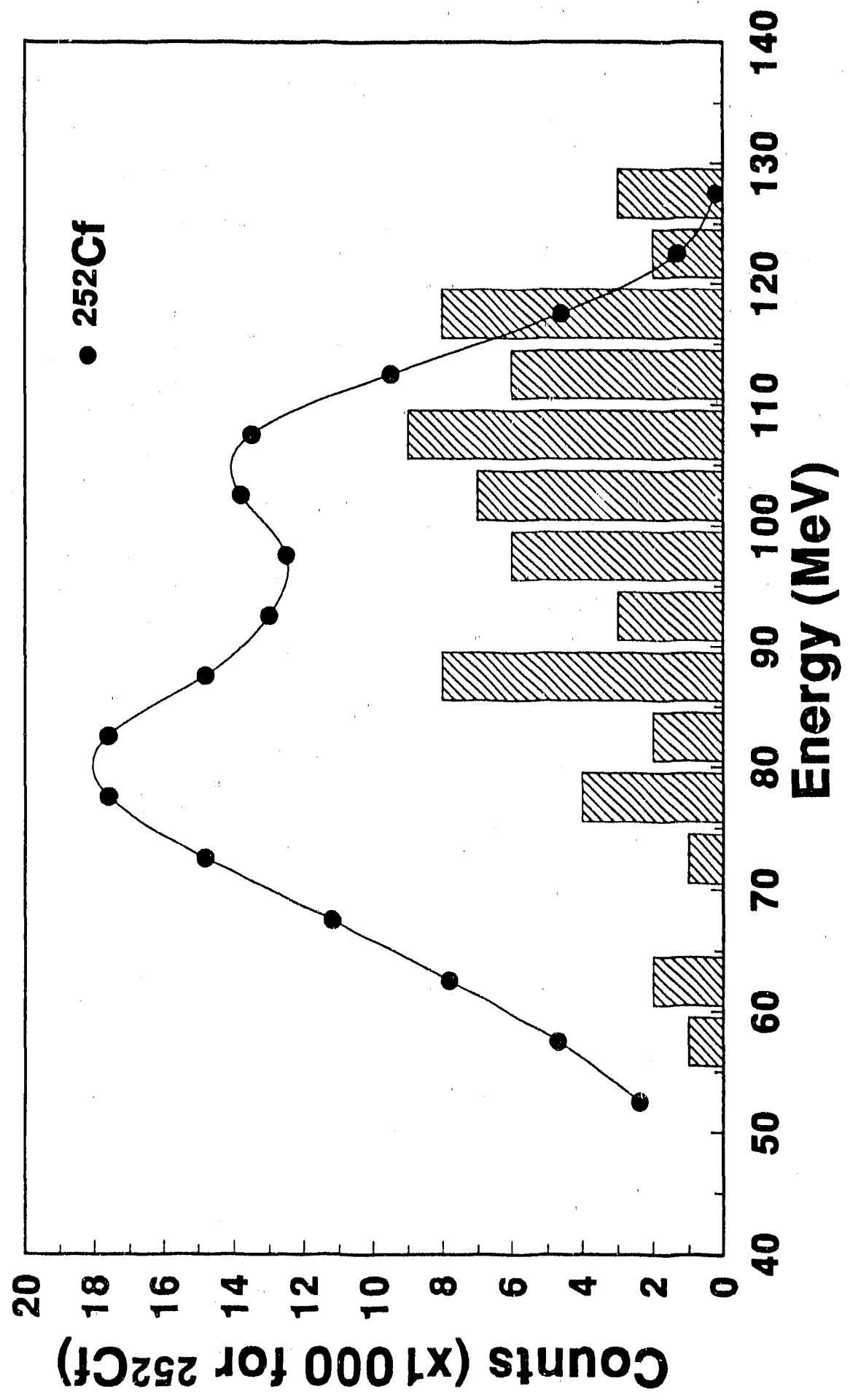

Figure 18: Energy histogram of the $62^{261} \mathrm{Lr}$ fission events. The smooth curve represents the energy calibration from ${ }^{252} \mathrm{Cf}$. 


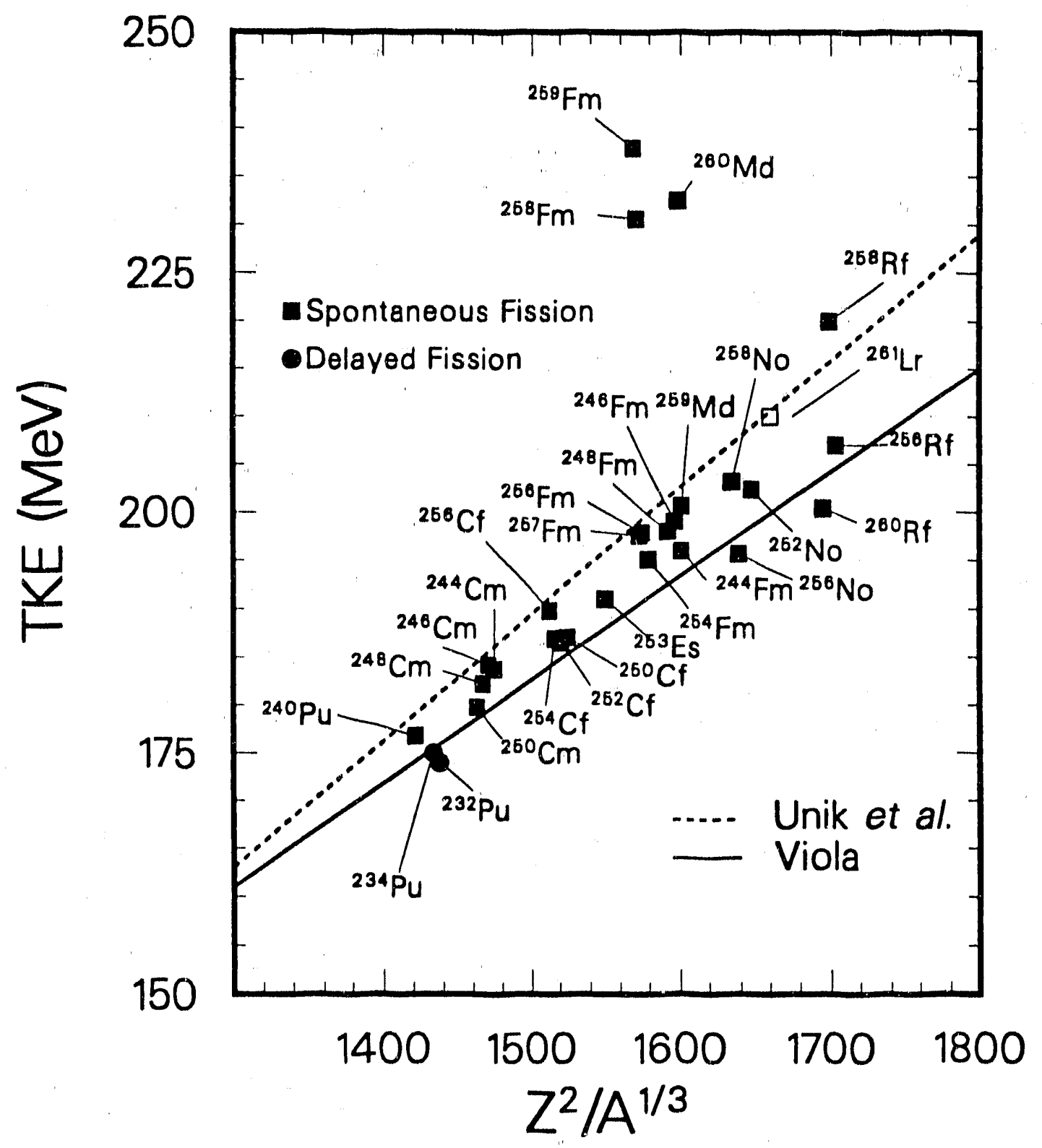

Figure 19: Plot of TKE vs. $Z^{2} / \mathrm{A}^{1 / 3}$. The dashed line is the linear fit of Unik et. al. [UNI74]; the solid line is from Viola [VIO66]. 
calculation. A $\log _{\mathrm{ft}}=6.4$ results from the proposed $14 \%{ }^{261} \mathrm{Rf}$ EC branch, when calculated using the nomogram in [LED78]. This $\log _{\mathrm{ft}}$ value is quite close to the value of 6.0 that was used to estimate the EC branch. This EC branch, if it really is such, would not have been detected in the discovery experiment for ${ }^{261} \mathrm{Rf}$, as the experiment was designed to look for short-lived activities. There was also a problem from the fissioning species that were produced directly, in particular ${ }^{256} \mathrm{Fm}$, which would mask this activity.

\section{Production of ${ }^{262} \mathrm{Lr}$}

The production of ${ }^{262} \mathrm{Lr}$ was studied in order to help determine the magnitude of compound-nucleus $(\mathrm{pxn})$ type reactions relative to compound-nucleus $((x+1) n)$ reactions in this region of the chart of the nuclides. The reaction used was ${ }^{248} \mathrm{Cm}\left({ }^{18} \mathrm{O}, \mathrm{p} 3 \mathrm{n}\right)^{262} \mathrm{Lr}$, with an $18 \mathrm{O}$ energy on target of $93 \mathrm{MeV} .{ }^{262} \mathrm{Lr}$ cannot be produced by electron-capture from ${ }^{262} \mathrm{Rf}$, which would be expected to have a very short SF half-life, and in fact, a 52 ms spontaneous fission activity has been assigned to this isotope [SOM85]. The 52-ms half-life is much too short for an electron capture process to compete with the fission process. Assuming a $\log _{\mathrm{fl}}>4.0,262 \mathrm{Rf}$ is expected to have an electron-capture half-life greater than 4 minutes. This relatively long half-life comes about primarily from the low $\mathrm{Q}_{\mathrm{EC}}$, which is predicted to be about $0.6 \mathrm{MeV}$. This half-life estimate would give an EC branch of $2 \times 10^{-4}$, the lowest value that it could have. This is merely to show that an EC branch is not likely to exist in the decay of ${ }^{262} \mathrm{Rf}$. It is therefore an ideal case to see if the $700 \mathrm{pb} \sigma$ for the $\mathrm{p} 4 \mathrm{n}$ reaction is reasonable.

The recoiling ${ }^{262} \mathrm{Lr}$ atoms produced in this reaction were caught in Au catcher foils. A series of six to seven hour bombardments was used, for a total of nine irradiations. The catcher foils were removed from the target system following the end of the bombardment, were cut from their support rings and placed in a $15 \mathrm{ml}$ centrifuge tube. Two drops of concentrated $\mathrm{HCl}$ and one drop of $\mathrm{HNO}_{3}$ were added to the tube, which 
was then heated in a hot water bath to speed the dissolution of the foil. The resulting solution was then placed on the top of an anion-exchange resin column. The column was 2-mm i.d. by $50-\mathrm{mm}$ in length. The activity was pushed onto the resin using $\mathrm{N}_{2}$ gas pressure. The actinide fraction was stripped from the column with concentrated $\mathrm{HCl}$. The eluate was collected in a glass planchette, which was placed beneath a heat lamp. After the concentrated $\mathrm{HCl}$ was evaporated, a small amount of $1 \mathrm{M} \mathrm{HCl}$ was added to the planchette and taken to dryness. The sample was picked up with $95 \mu l$ of $0.05 \mathrm{M} \alpha$-HIB, without any $\mathrm{pH}$ adjustment. This was injected into ACCESS and the Lr fraction was eluted from the column as before.

The decay curve for the 80 observed fission events is shown in figure $20 .{ }^{261} \mathrm{Lr}$ was also produced in this reaction, as is seen from the data fit. A long background component was used to account for the background on the detectors. This is necessary due to the long counting intervals used, which were at least 40 hours total time, to allow the 3.6-h ${ }^{262} \mathrm{Lr}$ to decay entirely. Taking into account the total chemical efficiency of $40 \%$, the $80 \%$ SF detector geometry, and the average saturation factor for a 6.5 hour bombardment, a production cross section of $240 \pm 24 \mathrm{pb}$ is calculated.

Compared to the $700 \mathrm{pb}$ cross section for the production of ${ }^{261} \mathrm{Lr}$, this seems to be somewhat low for a $(\mathrm{p} 3 \mathrm{n})$ out reaction. However, there are two factors which must be accounted for. First is that ${ }^{261} \mathrm{Lr}$ can be produced by EC in ${ }^{261} \mathrm{Rf}$. The second factor has to do with the bombarding energy. A touching splieres calculation, using a radius parameter of 1.4 , of the Coulomb barrier for ${ }^{18} \mathrm{O}+{ }^{248} \mathrm{Cm}$ gives a lab-frame barrier of 94.4 MeV. The energy on target for this reaction was $93 \mathrm{MeV}$, or below the Coulomb barrier when it is calculated in this manner. Since there was no evidence for the production of ${ }^{262} \mathrm{Lr}$ at $98 \mathrm{MeV}$ on target, it was necessary to drop the $18 \mathrm{O}$ energy to as low a level as possible. The excitation function for the $\mathrm{p} 3 \mathrm{n}$ reaction channel should have a higher maximum cross section than the $\mathrm{p} 4 \mathrm{n}$ reaction channel, in the absence of the Coulomb barrier. This would be analogous to the observations [ESK71][ESK73] 


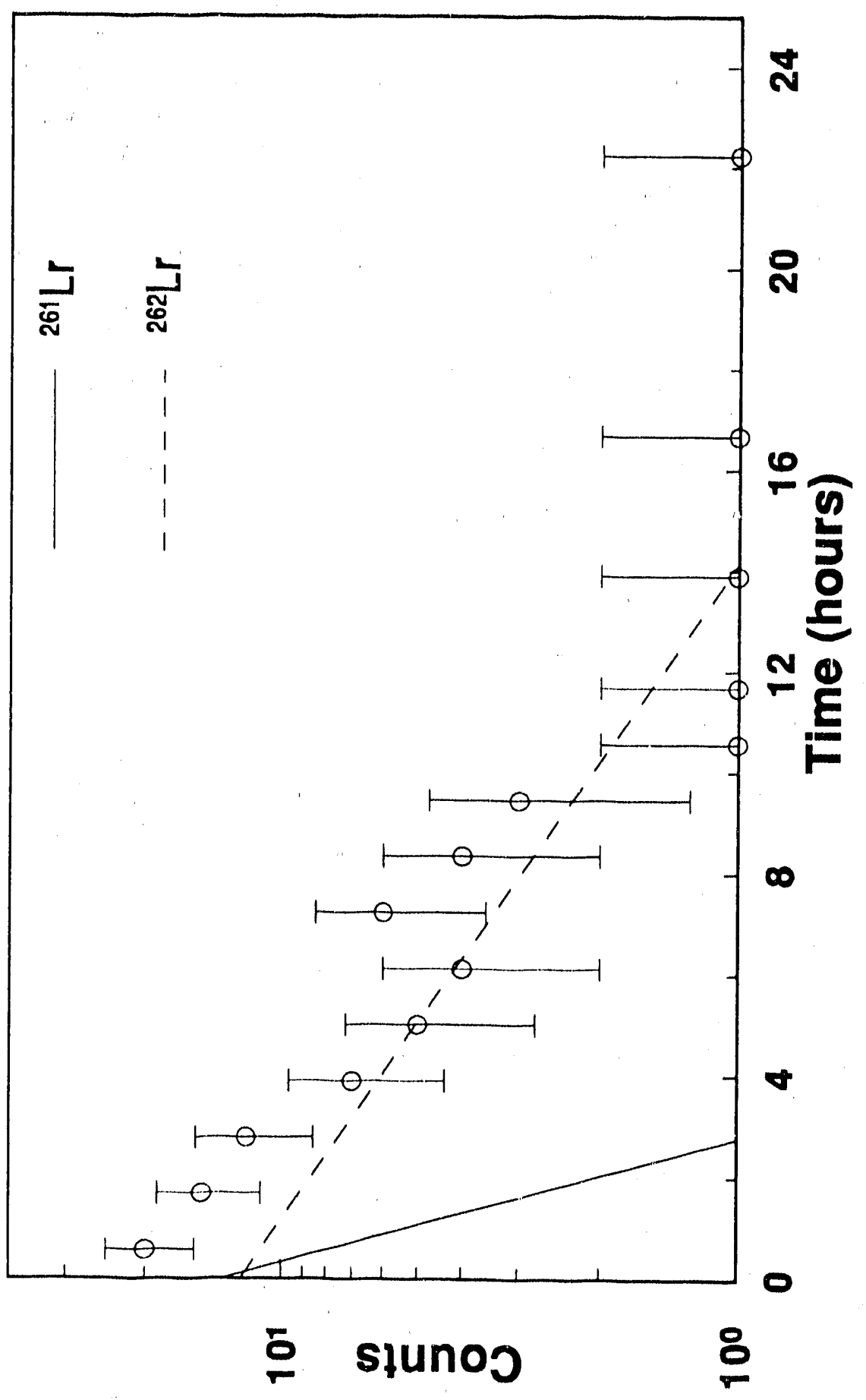

Figure 20: Decay curve for the fission events for the ${ }^{262} \mathrm{Lr}$ experiments. 
[SOM85] that, for projectiles heavier than ${ }^{11} \mathrm{~B}$, the compound-nucleus, $\mathrm{CN},(5 n)$ reactions have higher maximum cross sections than $\mathrm{CN}(4 \mathrm{n})$ reactions. These are comparable to the $\mathrm{CN}(\mathrm{p} 4 \mathrm{n})$ and $\mathrm{CN}(\mathrm{p} 3 \mathrm{n})$ reactions, respectively, as the same number of particles are being evaporated from the compound nucleus. Because of the Coulomb barrier, this 'larger' cross section part of the excitation function will not be accessible, and, therefore, a smaller cross section will be observed. As for the EC branch in $261 \mathrm{Rf}$, these experiments indicate that a value of $14 \%$ must be taken as an upper limit only, as the pxn reaction channels have been shown to have cross sections that are on the same order as the EC branch is expected to be. These results will be discussed later in the chapter V. 


\section{Chapter IV: Hahnium Experiments}

\section{A. Adsorption Studies}

${ }^{262} \mathrm{Ha}$ is the longest-lived isotope of this element, having a half-life of $34 \pm 4$ seconds. Procedures aimed at studying the chemical properties of this element must therefore be quite rapid. The source that is prepared from the chemical procedure must also be very clean, so as not to degrade the resolution of the alpha-spectra. Clean samples are an absolute necessity when working with very small numbers of atoms, as any mass in the sample can degrade the energy of the emitted alpha particles sufficiently so that $\mathrm{Ha}$, or contaminants in the samples, could be mistaken for each other.

${ }^{262} \mathrm{Ha}$ has decay properties that allow the definitive identification of this isotope. The decay scheme for ${ }^{262} \mathrm{Ha}$ and its alpha decay daughter, ${ }^{258} \mathrm{Lr}$, which has a half-life of 4.3 \pm 0.5 seconds, is shown in figure 21 . Since each event that is recorded by the RAGS system is recorded with the time that it occurred, the parent ${ }^{262} \mathrm{Ha}$ alpha decays can be correlated in time with the ${ }^{258} \mathrm{Lr}$ daughter decays. By observing the proper number of $\alpha-$ $\alpha$ correlated pairs, it can be proved that $262 \mathrm{Ha}$ was present in the samples.

Group 5 elements have been shown to adhere quite readily to glass and other surfaces under a variety of conditions [AHR76][TRA76][WEI81]. This characteristic adsorption property was employed to perform the first wet chemistry on Ha. Undesirable reaction contaminants, such as the actinide elements, were found not to adhere to the surfaces under the conditions used. The proposed procedures were tested using homologs of $\mathrm{Rf}$ and $\mathrm{Ha}$. Tracer activities of the group 5 elements $\mathrm{Nb}$ and $\mathrm{Ta}$ were formed by interactions of ${ }^{4} \mathrm{He}$ with natural $\mathrm{Y}$ and Lu targets, respectively. Tracer activities of the group 4 elements $\mathrm{Zr}$ and $\mathrm{Hf}$ were formed by interactions of ${ }^{4} \mathrm{He}$ with targets consisting of natural $\mathrm{Sr}$ and $\mathrm{Yb}$, respectively. Several adsorption conditions were tried, and the results are tabulated in Table IV. It was necessary to study several substrates to find the best 


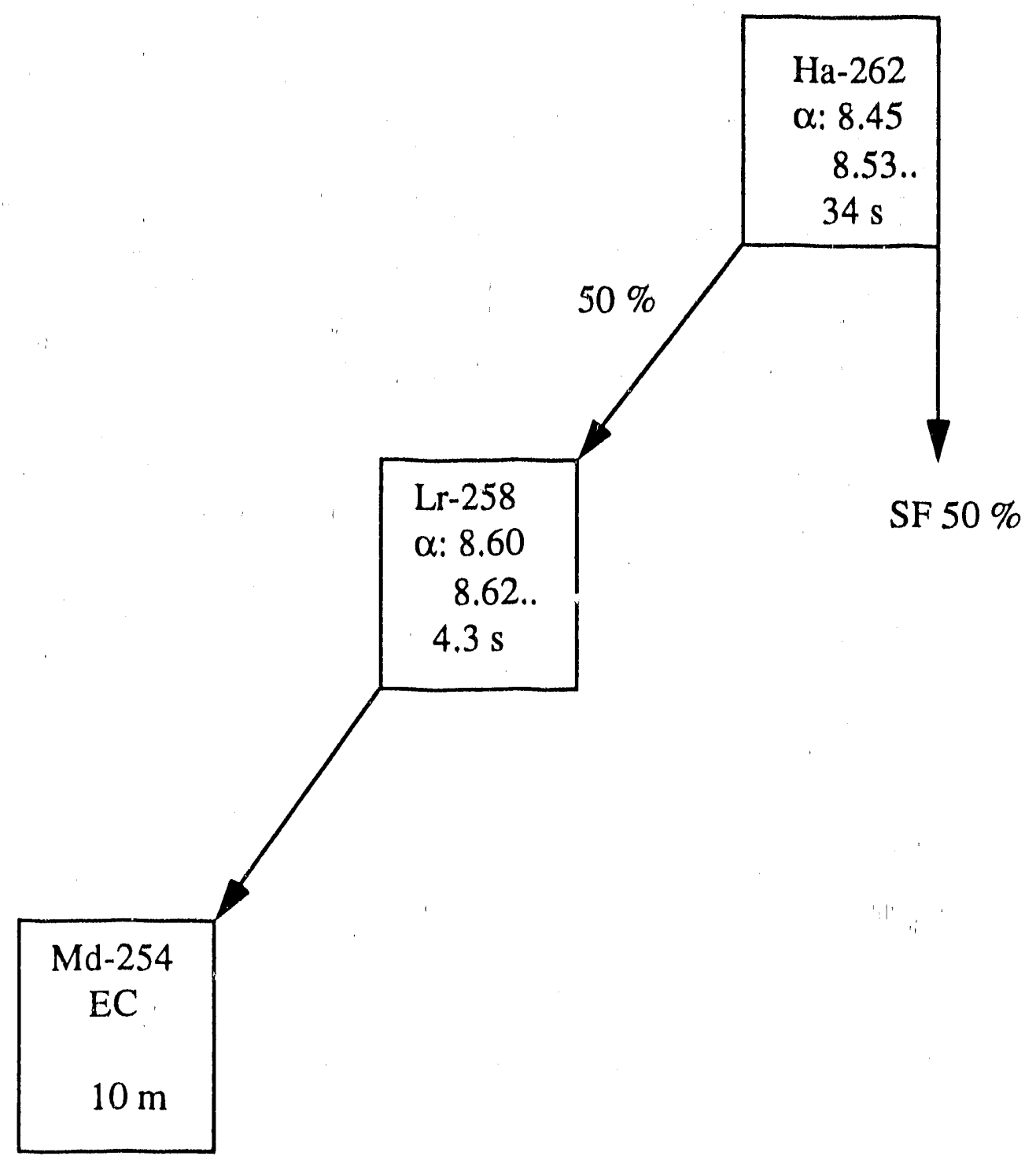

Figure 21: ${ }^{262} \mathrm{Ha}$ decay chain 
conditions for this experiment. Due to the low production rate for ${ }^{262} \mathrm{Ha}$, it is necessary to choose conditions where the highest adsorption yields can be attained. From the results it is observed that the best adsorption of the group 5 elements occurs from two fumings with concentrated $\mathrm{HNO}_{3}$ on a glass surface. These were the conditions that were chosen for the $\mathrm{Ha}$ experiments.

Table IV: Adsorption yields for some Group 4 and 5 Elements

\begin{tabular}{|c|c|c|c|c|c|c|c|}
\hline $\begin{array}{c}\text { Surface } \\
\text { Treatment }\end{array}$ & $\begin{array}{c}\text { Glass } \\
1 \mathrm{xHNO}_{3}\end{array}$ & $\begin{array}{c}\text { Glass } \\
2 \mathrm{xHNO}_{3}\end{array}$ & $\begin{array}{c}\text { Glass } \\
1 \times \mathrm{HCl}\end{array}$ & $\begin{array}{c}\mathrm{Pt} \\
1 \times \mathrm{HNO}_{3}\end{array}$ & $\begin{array}{c}\mathrm{Pt} \\
1 \times \mathrm{HCl}\end{array}$ & $\begin{array}{c}\text { Teflon } \\
1 \mathrm{xHNO}_{3}\end{array}$ & $\begin{array}{c}\text { Teflon } \\
1 \times \mathrm{HCl}\end{array}$ \\
\hline $\begin{array}{c}\text { Group 4 } \\
\mathrm{Zr}\end{array}$ & $4 \%$ & $0 \%$ & $30 \%$ & -- & -- & -- &.- \\
$\mathrm{Hf}$ & $28 \%$ & $11 \%$ & $59 \%$ & $27 \%$ & -- & $5 \%$ &.- \\
\hline Group 5 & & & & & & & \\
$\mathrm{Nb}$ & $35 \%$ & $42 \%$ & $28 \%$ & $22 \%$ & -- & $4 \%$ & $5 \%$ \\
$\mathrm{Ta}$ & $74 \%$ & $80 \%$ & $76 \%$ & $60 \%$ & $58 \%$ & $35 \%$ & $10 \%$ \\
\hline
\end{tabular}

${ }^{262} \mathrm{Ha}$ was produced using the ${ }^{249} \mathrm{Bk}\left({ }^{18} \mathrm{O}, 5 \mathrm{n}\right){ }^{262} \mathrm{Ha}$ reaction, with a production cross section of $5 \mathrm{nb}$. The detection rate that is expected for this experiment can be calculated using this production rate, the collection time, $60 \mathrm{sec}$, the length of time needed to perform the chemistry, $51 \mathrm{sec}$, and the detector geometry, 35\%. When these factors are calculated, a data rate of one detected event per three experiments, uncorrected for chemical yield, results. Therefore, to gain meaningful results for this experiment it is necessary to repeat the procedure many times. A total of 801 separate experiments were performed, with a total of 52 events, 26 fissions and $26 \alpha$-particles, being observed. A summed $\alpha$-spectrum from the experiments is shown in figure 22 , representing all of the data from these 801 separate experiments. In this spectrum it is important to notice the 


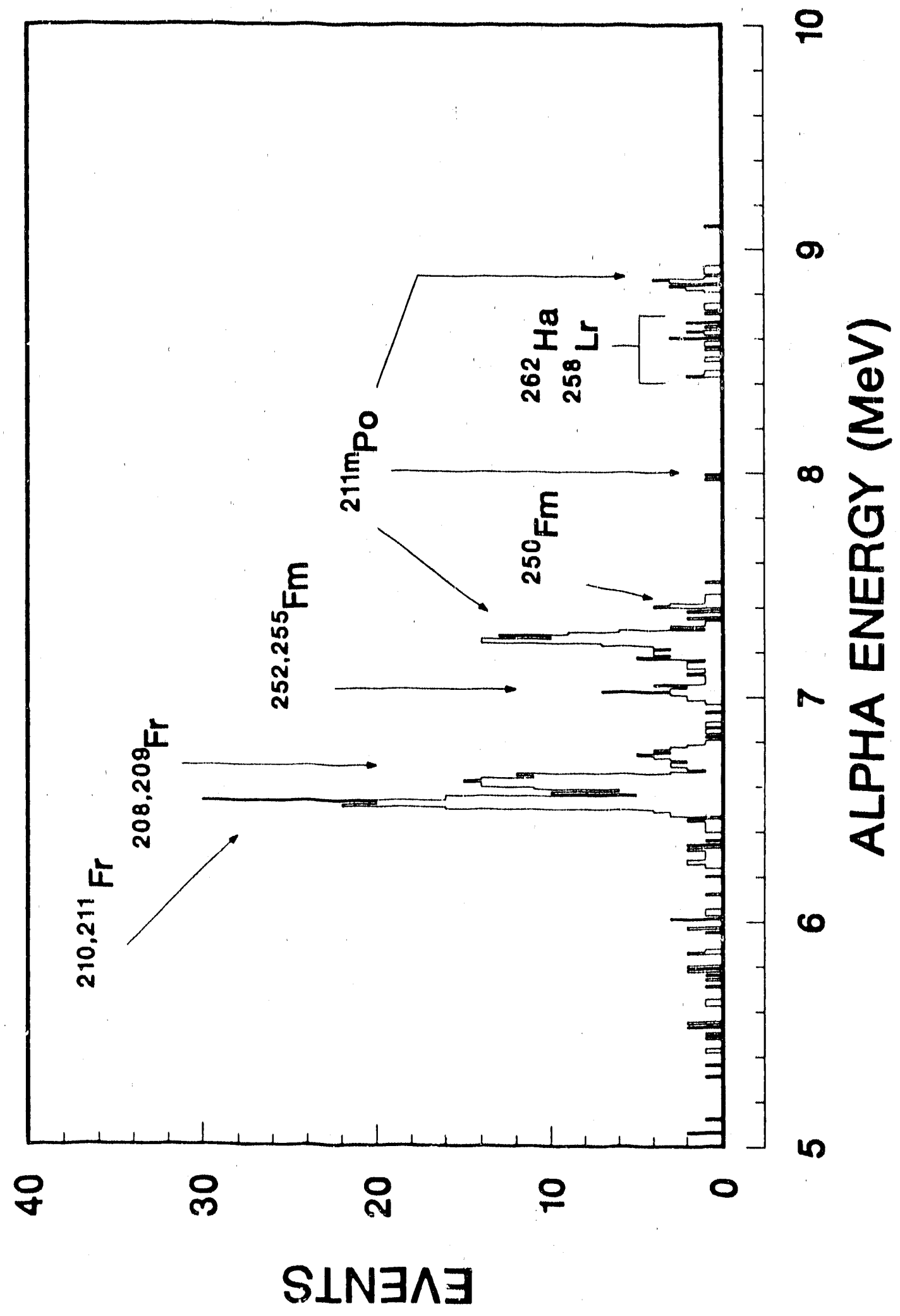

Figure 22: Summed $\alpha$ spectrum from the 801 adsorption experiments 
verv low leve!s of contamination from the actinide elements. The amount of Fm that is present in the spectrum represents decontamination from the actinides by a factor of 400 . The amount of Fm produced in this reaction is measured directly from the gas jet yield, which was checked periodically during the experiment by flaming an untreated sample to remove the $\mathrm{KCl}$ aerosols and then placing the sample on one of the $\mathrm{Si}(\mathrm{Au})$ surface barrier detectors. The efficiency of the gas jet was typically about $80 \%$.

Based on these results it is possible to conclude that in this system, Ha shows chemical properties similar to the homologous group 5 elements $\mathrm{Nb}$ and $\mathrm{Ta}$. However, the results of this experiment do not tell anything about the expected or predicted trend in the halide complexation of $\mathrm{Ha}$.

\section{B. Extraction Studies}

The second series of experiments performed on $\mathrm{Ha}$ were solvent extraction studies. The solvent extraction technique can be useful for the study of very short-lived species. (See p. 27) Single step solvent extraction procedures can be carried out in a short period of time provided that the kinetics of the extraction are fast, and good phase contact followed by a rapid separation of the two phases can be achieved. The organic solvents that are used for solvent extraction quite often have low boiling points, and can thus be evaporated quite quickly. The choice of solvent and/or extractant can often be such that after evaporation little or no residue is left hehind, and if there is residue, it can usually be flamed away.

An industrial procedure for the separation of $\mathrm{Nb}$ and Ta [WER54] was adapted for use as a micro-scale experiment. The separation of $\mathrm{Nb}$ and $\mathrm{Ta}$ can be achieved by the selective extraction of singly charged anionic species into methylisobutyl ketone (MIBK) from a mixed $\mathrm{HCl} / \mathrm{HF}$ acid solution. The basis for this separation is the extractability of Ta into MIBK from a mixed acid system. At low HF concentrations, Ta can be 
selectively extracted into MIBK, while $\mathrm{Nb}$ will remain in the aqueous phase. Other authors have reported similar results on macro-scale experiments [MIL55][STE53] [WAT57].

Raman studies have been performed to determine the $\mathrm{Nb}$ and $\mathrm{Ta}$ fluoride species that exist in aqueous solution [KEL63][KEL66]. It has been shown by Keller and ChethamStrode [KEL66] that the predominant Ta species in $\mathrm{HF}$ solutions are $\mathrm{TaF}_{6}^{-}$and $\mathrm{TaF}_{7}{ }^{2-}$. At high $\mathrm{HF}$ concentration, the $\mathrm{TaF}_{6}-$ species has been found to predominate. With $\mathrm{NH}_{4} \mathrm{~F}$ present in high concentration, the $\mathrm{TaF}_{7}{ }^{2-}$ species predominates. For the $\mathrm{Nb}$ systems, the predominant species in solution is the $\mathrm{NbOF}_{5}{ }^{2-}$ ion. Only at high $\mathrm{HF}$ concentration will the pure fluoride $\mathrm{NbF}_{6}{ }^{-}$species be formed. These results confirm earlier measurements which were performed non-spectrophotometrically [BUS59][NIK59].

Marcus and Kertes have pointed out that oxygen-containing organic solvents will preferentially extract singly-charged anionic species [MAR69A]. MIBK is such an oxygen containing organic solvent. This extraction property adequately explains the observed behavior that Ta will extract with very small amounts of HF present, whereas the HF concentration must be quite high before $\mathrm{Nb}$ will begin to extract. Thus it is the $\mathrm{MF}_{6}{ }^{-}$species that is being extracted by this solvent, and not the doubly charged oxyfluoride anion or the $\mathrm{MF}_{7}{ }^{2-}$ species.

The trend of forming the pure halide species more readily as one moves down the group 5 elements from $\mathrm{Nb}$ to $\mathrm{Ta}$ might be predicted to continue to $\mathrm{Ha}$. This experiment was designed to look for such behavior in $\mathrm{Ha}$. This experiment was also the first look at the aqueous halide complexing ability of $\mathrm{Ha}$.

Preliminary experiments were performed to determine if the industrial procedure could be adapted for use as a micro-scale experiment. The main concerns about the procedure were kinetics and efficiency. As has been mentioned many times before, all procedures aimed at the study of the chemical properties of Ha must be performed in the shortest time possible, due to the 34 second half-life of the longest-lived isotope, ${ }^{262} \mathrm{Ha}$. 
${ }^{182} \mathrm{Ta}, \mathrm{t}_{1 / 2}=114.43 \mathrm{~d}$, tracer was used to test the extraction procedure. With the small volumes used for this experiment, a $10 \mu \mathrm{l}$ aqueous phase and a $20 \mu \mathrm{l}$ organic phase, mechanical mixing is not possible, as it does not adequately mix the two phases. However, when the centrifuge tube is placed in the ultrasonic stream from an ultrasonic humidifier, complete phase mixing is achieved almost instantaneously. It was found that about $75 \%$ of the Ta tracer was extracted into the MIBK layer after only a few seconds of mixing. The lower yield relative to the yields that were reported in the literature was due primarily to incomplete removal of the $20 \mu \mathrm{M}$ MIBK phase. A second set of test experiments was performed using ${ }^{249} \mathrm{Cf}$ tracer to determine the behavior of the $3+$ actinides in this system. As expected, $\mathrm{Cf}$ did not extract into the MIBK layer, since the actinide ions do not form a singly charged anionic species under these conditions.

The conditions for this experiment were chosen such that it was specific for Ta and Ta-like species. There were two main reasons for this, the first being that since the separations were performed using glass centrifuge tubes, the HF concentration must be kept low. Secondly, it was found that when the acid concentration was raised above about $6 \mathrm{M}$, the interface between the organic and aqueous phase was no longer visible. A final set of tests was performed using $\mathrm{Pa}$, which has a $5+$ oxidation state, as do $\mathrm{Nb}$ and $\mathrm{Ta}$, and is chemically similar to $\mathrm{Nb}$. $\mathrm{Pa}$ was also found not to extract under the conditions used.

335 separations from one minute collections were performed on $\mathrm{Ha}$ using this procedure. Based upon the observed yields from the adsorption experiments, 14 SF decays and 14 alpha decays, including two correlated parent-daughter pairs should have been observed if $\mathrm{Ha}$ was exhibiting eka-Ta-îke chemical properties. In the energy range of 8.4-8.7 MeV, no alpha events were observed, nor were any fission events observed, indicating that $\mathrm{Ha}$ had not been extracted by MIBK under these conditions.

In summary, it has been demonstrated that the isotope assigned to ${ }^{262} \mathrm{Ha}$ displays group 5 characteristics, as was shown in the adsorption experiments. It is also shown that 
Ha does not exhibit eka-Ta-like chemical characteristics in these solvent extraction studies. These seemingly contradictory results can be explained by considering the chemical species that might be involved in these studies. In the adsorption experiments, no halide ions were present, so presumably some sort of an oxide compound is being formed, although the specific chemical form has not been studied. This species then readily adheres to the glass surface, as has already been found to be characteristic of Group 5 elements.

The solvent extraction experiments were designed to determine only if $\mathrm{Ha}$ was behaving as eka-Ta. From the observation that Ha had not been extracted by MIBK, it is obvious that a non-extractable anionic species has been formed. This could either be a pure halide complex, $\mathrm{HaF}_{7}{ }^{2-}$, or an oxygen-containing complex, $\mathrm{HaOF}_{5}{ }^{2-}$, as has been found with $\mathrm{Nb}$. It is of interest to note that $\mathrm{Pa}$, which shows Group 5 chemical characteristics, is not extracted by MIBK under these conditions. Pa forms complexes of the type $\mathrm{PaOF}_{5}{ }^{2-}$. Forming the pure halide species seems to be unlikely; however, this possibility cannot te excluded by these experiments. 


\section{Chapter V: Discussion}

\section{A: Chemistry of Lawrencium}

The results of the TTA extraction experiments showed that $260 \mathrm{Lr}$ has extraction properties similar to the other $3+$ actinide ions that were produced in the reaction, and as such is chemically similar to ${ }^{256} \mathrm{Lr}$ [SIL70]. 'This constituted the first chemical proof that the activity assigned to $260 \mathrm{Lr}$ does indeed belong to $\mathrm{Lr}$.

Table IV: 3+ Crystal Ionic Radii

\begin{tabular}{|c|c|c|c|c|c|c|}
\hline Element & $\begin{array}{c}\text { Radius } \\
(\mathrm{nm}) \\
\text { [DAV85] }\end{array}$ & $\begin{array}{c}\text { Spacing } \\
\mathrm{r}^{-}{ }^{-}(\mathrm{z}-1)\end{array}$ & $\begin{array}{c}\text { Radius } \\
(\mathrm{nm}) \\
\text { [BRA86] }\end{array}$ & $\begin{array}{c}\text { Spacing } \\
\mathrm{r}^{-\mathrm{r}}(\mathrm{z}-1)\end{array}$ & $\begin{array}{c}\text { Radius } \\
(\mathrm{nm}) \\
\text { exp. }\end{array}$ & $\begin{array}{c}\text { Spacing } \\
\mathrm{r}_{\mathbf{z}^{\mathrm{r}}(\mathrm{z}-1)}\end{array}$ \\
\hline $\mathrm{Am}$ & 0.0980 & & 0.0976 & & & \\
\hline $\mathrm{Cm}$ & 0.0970 & 0.0010 & 0.0960 & 0.0016 & & \\
\hline $\mathrm{Bk}$ & 0.0955 & 0.0015 & 0.0945 & 0.0015 & $0.0955^{\mathrm{a}}$ & \\
\hline $\mathrm{Cf}$ & 0.0945 & 0.0010 & 0.0932 & 0.0013 & $0.0942^{\mathrm{a}}$ & 0.0013 \\
\hline $\mathrm{Es}$ & 0.0934 & 0.0011 & 0.0920 & 0.0012 & $0.0928^{\mathrm{a}}$ & 0.0014 \\
\hline Fm & 0.0922 & 0.0012 & 0.0909 & 0.0011 & $0.0911^{\mathrm{b}}$ & 0.0017 \\
\hline $\mathrm{Md}$ & 0.0912 & 0.0010 & 0.0898 & 0.0011 & $0.0896^{\mathrm{b}}$ & 0.0015 \\
\hline $\mathrm{No}$ & 0.0902 & 0.0010 & 0.0889 & 0.0009 & & \\
\hline $\mathrm{Lr}$ & 0.0896 & 0.0006 & 0.0880 & 0.0009 & $0.0886^{\mathrm{c}}$ & $0.0010^{\mathrm{d}}$ \\
\hline
\end{tabular}

aRadii from electron diffraction studies [HAI73] bRadii from elution positions [CHO56]

'This work d Lr-Md spacing 
Bratsch and Lagowski [BRA86] predicted a value of the ionic radius that is 0.0006 $\mathrm{nm}$ smaller than the radius determined in this study, or a difference of less than one percent. These values are both based upon an ionic radius of $\mathrm{Er}^{3+}$ of $0.0881 \mathrm{~nm}$ as reported by Templeton and Dauben [TEM54]. However, this is little more than coincidental, as other actinide radii predicted by the same authors do not agree well with the experimentally observed values. The $\mathrm{Bk}^{3+}, \mathrm{Cf}^{3+}$, and $\mathrm{Es}^{3+}$ radii determined by electron diffraction [HAI72] are larger than the predicted values by about $0.0010 \mathrm{~nm}$. The differences between the observed and calculated $\mathrm{Fm}^{3+}$ and $\mathrm{Md}^{3+}$ radii are smaller. Table IV displays the theoretical and experimental values for most of the heavy actinide $3+$ ions. For additional comparison, the values predicted by David et. al. [DAV85] are also included.

The most interesting result of this ionic radius determination is the spacing between the $\mathrm{Md}^{3+}$ and $\mathrm{Lr}^{3+}$ ions. The relative elution positions of these two ions gives a spacing of about $0.0010 \mathrm{~nm}$, which, when compared to the spacings at the end of the lanthanide series, is abnormally small for a two $\mathrm{Z}$ spacing. Ho and $\mathrm{Er}$ are next to each other in the periodic table, whereas Md and $\mathrm{Lr}$ are separated by No. The spacing between $\mathrm{Ho}$ and $\mathrm{Er}$ is $0.0013 \mathrm{~nm}$, and the typical two $\mathrm{Z}$ spacing at the end of the series is $0.0022 \mathrm{~nm}$. The ionic radius of $\mathrm{Lr}^{3+}$ has been determined to be larger than had been expected. This gives evidence that the actinide contraction may be less pronounced than the well established lanthanide contraction. This unexpectedly large ionic radius makes the determination of the $\mathrm{No}^{3+}$ ionic radius very interesting. Nobelium, as was mentioned earlier, exists in aqueous solution as a $2+$ cation. Attempts to determine this radius are described in Appendix B.

One method of explaining this larger radius is the influence of relativistic effects. As mentioned before, direct relativistic effects will have the largest effect on low angularmomentum quantum number electrons, such as $s$ and $p$ electrons. These electrons will be accelerated to relativistic velocities as they pass near the nucleus in a heavy atom. The 
mass increase that they experience has the end result of decreasing the radius of the electronic orbit, and thus shrinks the electron shell. This direct effect will produce secondary effects, namely, as the $s$ and p electron shells shrink in size, their effectiveness in screening the nuclear charge will increase [PYY79]. This increased screening will then cause electrons in the $d$ and $f$ shells to have lower binding energies, and therefore extend radially farther from the nucleus than in the absence of relativistic effects. With the much larger charges of the actinides as compared with the lanthanides the corresponding relativistic effects are expected to be much larger. From the data it is not possible to determine whether $\mathrm{Lr}$ is displaying more relativistic effects than the rest of the actinides or not. Relativistic effects may be responsible for the heavy actinide ions with incumplete 5f electron shells being larger than expected when compared to $\mathrm{Lr}^{3+}$, which has a complete $5 \mathrm{f}$ electron shell, resulting in the small changes in radii observed at the end of the actinide series. The other possibility is that L $\mathrm{r}^{3+}$ is large when compared to the rest of the $3+$ ions at the end of the actinide series. With these experiments, it is not possible to determine which of these is the correct answer.

\section{B: Isotopes of Lawrencium}

The production cross sections of ${ }^{261} \mathrm{Lr}$ and ${ }^{262} \mathrm{Lr}$ from the interactions of ${ }^{18} \mathrm{O}$ with $248 \mathrm{Cm}$ have been determined as $700 \mathrm{pb}$ and $240 \mathrm{pb}$, respectively in Chapter III, p 48 and

51. There was the possibility that ${ }^{261} \mathrm{Lr}$ could have been produced by an electron-capture decay branch in ${ }^{261} \mathrm{Rf}$. However, with the observed cross section for the production of ${ }^{262} \mathrm{Lr}$ of $240 \mathrm{pb}$, it seems likely that ${ }^{261} \mathrm{Lr}$ is only produced by the nuclear reaction, for reasons which were discussed in greater detail at the end of chapter III. This does set an upper limit on the EC branch in ${ }^{261} \mathrm{Rf}$ of $14 \%$, arrived at by comparing the relative production cross sections for the parent and daughter nuclei.

It has also been demonstrated by these results that these longer-lived isotopes of $\mathrm{Lr}$ 
can be produced without using an ${ }^{254}$ Es target, for which future supplies still remain in question. The production of these two longer-lived isotopes permits more complex chemical experiments to be performed to examine different facets of the chemistry of the final member of the actinide series.

\section{C: Chemistry of Hahnium}

The experiments performed on Ha have shown that this element does display Group 5 chemical characteristics, as shown in particular by the adsorption experiments. It has also been demonstrated that the chemical properties in the MIBK extraction experiments are not similar to $\mathrm{Ta}$. This indicates that $\mathrm{Ha}$ is not following the trend of forming pure halide complexes that is noted in the transition from $\mathrm{Nb}$ to $\mathrm{Ta}$.

Ha has been chemically determined by these studies to belong in the Group 5 series in the periodic system, even though a simple extrapolation of the chemistry of this series to Ha cannot be made.

One other possibility for explaining the non-extraction of $\mathrm{Ha}$ by MIBK is that Ha may form a relativistically stabilized $3+$ cation in solution. This would then be analogous to an actinide $3+$ ion, which will not form an extractable complex under these conditions. Relativistic calculations by Desclaux [DES73] indicate that $\mathrm{Ha}^{3+}$ would only exist under strongly reducing conditions, so this explanation seems unlikely.

\section{D: Future}

The future of heavy element studies is dependent on two main factors. First is the half-lives of the isotopes available for study. As new longer-lived isotopes are discovered, it becomes possible to devise new experiments to explore different facets of an element's chemical properties. Experiments that could not be performed can now be 
considered with the discovery of ${ }^{261} \mathrm{Lr}$ and ${ }^{262} \mathrm{Lr}$. The second main consideration is the time that is necessary for a process to be performed. Most classical chemical methods cannot be used in the heavy element region. New processes that can be performed in a very short time must be developed. It has been shown in the current study that in some cases large-scale experiments can be adapted for use on a micro-scale. Gas-phase experiments are also possible.

Perhaps the most important limiting factor in studying the chemical properties of the heaviest elements is the availability of target materials suitable for the production of isotopes for study. In this work it has been demonstrated that, at least for Lr studies, isotopes can be produced without using the more exotic target materials such as ${ }^{254} \mathrm{Es}$. Since there are alternative methods for producing isotopes of $\mathrm{Lr}$, the future of chemical studies of the last member of the actinide series looks bright indeed. 


\section{APPENDIX A: Attempts to Reduce Lr ${ }^{3+}$}

\section{A: Introduction}

As mentioned earlier, one aspect of relativistic effects might be the stabilization of the $\mathrm{Lr}^{1+}$ ion, which might exist if the stabilization of the $7 \mathrm{~s}$ electron pair against oxidation is strong enough. The existence of a $1+$ oxidation state would be a change from the chemistry of $\mathrm{Lu}$, in which the $\mathrm{Lu}^{1+}$ state is not known. As is also true in the lanthanide series, the $2+$ oxidation state in the actinide series is not the most stable oxidation state in solution, with the exception of nobelium. Few predictions of the $3+12+$ couple for $\mathrm{Lr}$ exist; however, Bratsch and Lagowski have predicted $\mathrm{E}^{\circ} \cong-2.6 \mathrm{~V}$ [BRA86]. This large potential makes the $2+$ oxidation state of $\mathrm{Lr}$ inaccessible in aqueous solution. Lanthanides in the $2+$ oxidation state that have $\mathrm{E}^{\circ}$ values more negative than $-2.3 \mathrm{~V}$ are known to spontaneously oxidize to the $3+$ state when put into aqueous solution [MIK89]. But perhaps the reduction potential of the $3+/ 1+$ couple is somewhat smaller than this, and $\mathrm{Lr}^{1+}$ might, therefore, be accessible in aqueous solution. At the time of this study, no previous work on the reduction of $\mathrm{Lr}^{3+}$ had been attempted. Finding an experimental value for the $\mathrm{Lr}^{3+}$ reduction potential would then add another data point for comparison with relativistic theories, as well as other theories used for predicting chemical properties of the heaviest elements.

A separation scheme using HDEHP, di-2-ethylhexylorthophosphoric acid, reversephase chromatography provides an excellent separation of atoms based on their charge state. 1+ cations can be removed from such a column with very dilute, i.e., $0.1 \mathrm{M}$ acid, usually $\mathrm{HNO}_{3}$. Higher charge state cations can be removed with higher acid concentrations. Reducing agents can also be introduced into the system, and used in the aqueous phase to reduce species that are held on the resin bed. The reducing agents will 
only affect the separation insofar as they can reduce a species to a lower charge state and allow it to be stripped off of the column using a lower acid concentration than would normally be necessary to remove it. This then affords a reasonably quick separation of the reduced $\mathrm{Lr}^{1+}$ from the rest of the unreduced actinide $3+$ fraction.

\section{B: Chemistry}

Hydroxylamine hydrochloride is one of the strongest reducing agents that can exist in aqueous solution, with an $\mathrm{E}^{\circ}=-1.87 \mathrm{~V}$. A solution of $0.06 \mathrm{M} \mathrm{NH}_{4} \mathrm{OH} \cdot \mathrm{HCl}$ in $0.3 \mathrm{M} \mathrm{HCl}$ was prepared as the eluiing and reducing agent by dissolving $6.95 \mathrm{~g}$ of solid $\mathrm{NH}_{4} \mathrm{OH} \cdot \mathrm{HCl}$ in $100 \mathrm{ml}$ of $0.3 \mathrm{M} \mathrm{HCl}$. An HDEHP column was prepared by the standard method [HOR69] of sorbing HDEHP onto sized nydrophobic Celite-535 from an acetone solution. The resin was prepared prior to treatment with the HDEHP by careful washing with $0.1 \mathrm{M} \mathrm{HCl}$ followed by exposure to dichlorodimethylsilane vapors under vacuum for several days. The prepared resin was packed to a height of $5 \mathrm{~cm}$ in a Pt tipped glass column, 2-mm i.d. by $70-\mathrm{mm}$ length, using a glass rod. A small glass wool plug was used at the bottom of the column to support the resin. Another plug was placed on top of the resin bed to facilitate loading activity onto the column. $\mathrm{N}_{2}$ gas pressure was used to push solutions through the column.

${ }^{260} \mathrm{Lr}$ atoms produced in the ${ }^{249} \mathrm{Bk}\left({ }^{18} \mathrm{O}, \alpha 3 \mathrm{n}\right){ }^{260} \mathrm{Lr}$ reaction were transported using the $\mathrm{He} / \mathrm{KCl}$ aerosol jet transport system to a collection site on top of the experimental cave. After a predetermined collection time, typically about five minutes, the $\mathrm{KCl}$ spot was removed from the $\mathrm{Pt}$ collection foil by dissolving it with a couple of drops of $0.3 \mathrm{M}$ $\mathrm{HNO}_{3}$. This solution was placed on top of the HDEHP column and pustied oris the resin bed. The column was then rinsed with a small volume of $0.3 \mathrm{M} \mathrm{HCl}$ to remove any $1+$ and $2+$ cations, and also $\mathrm{Ac}^{3+}$. The removal of $\mathrm{Ac}$ is critical, as ${ }^{223} \mathrm{Ac}$, which can be

formed from $\mathrm{Pb}$ impurities in the target, has decay properties such that it can easily be 
mistaken for ${ }^{260} \mathrm{Lr}$. As shown in figure 15 , the $8.026 \mathrm{MeV} \alpha$ particle from ${ }^{215} \mathrm{At}$ will be time correlated with the $7.31 \mathrm{MeV} \alpha$ particle from ${ }^{219} \mathrm{Fr}$. This correlation gives a characteristic signature of the parent, 2.2 minute ${ }^{223} \mathrm{Ac}$. Removal of the $2+$ ions is also necessary, as ${ }^{255}$ No can easily be misidentified as ${ }^{260} \mathrm{Lr}$ due to its similar half-life and alpha-particle energies. After washing, the reducing agent, $0.06 \mathrm{M} \mathrm{NH} \mathrm{NH}_{4} \mathrm{OH} \cdot \mathrm{HCl}$ in $0.3 \mathrm{M}$ $\mathrm{HCl}$ is run through the column. This will reduce and strip $\mathrm{Lr}^{1+}$, if it is present under these conditions. Finally the remaining $3+$ actinide fraction is stripped from the column with $3 \mathrm{M} \mathrm{HCl}$. Three separate fractions are collected. First is the Ac fraction, followed by the reducible fraction, and lastly the non-reduced actinide fraction. Each sample is dried on a hot plate, then flamed and placed on a Si-A tu surface barrier detector and assayed for $\alpha$ and spontaneous fission activity. A flowchart of the procedure is shown in figure 23.

\section{C: Results and Discussion of the HDEHP Experiment}

A total of 24 experiments were performed using the HDEHP aqueous phase system. Under these conditions $\mathrm{Lr}^{3+}$ was not found to be reducible. $8.03-\mathrm{MeV} \alpha$ events characteristic of ${ }^{260} \mathrm{Lr}$ were found in the 'non-reducible' actinide fraction. In addition, no ${ }^{223} \mathrm{Ac}$ events were found in the $\mathrm{Ac}^{3+}$ fraction, which indicates that there was very little $\mathrm{Pb}$ contamination in the target.

There arc two possibilities for the negative results. The first is that $\mathrm{Lr}^{3+}$ has a high enough reduction potential such that it is not reducible by hydroxylamine. The second reason has to do with the reducing agent itself. The half-cell reaction for hydroxylamine is as follows:

$$
\mathrm{N}_{2}+2 \mathrm{H}_{2} \mathrm{O}+4 \mathrm{H}^{+}+2 \mathrm{e}^{-} \Leftrightarrow=2 \mathrm{NH}_{3} \mathrm{OH}^{+} \quad \mathrm{E}^{0}=-1.87 \mathrm{~V}
$$


Pick up activity with 2 drops $0.3 \mathrm{M}$ Nitric acid

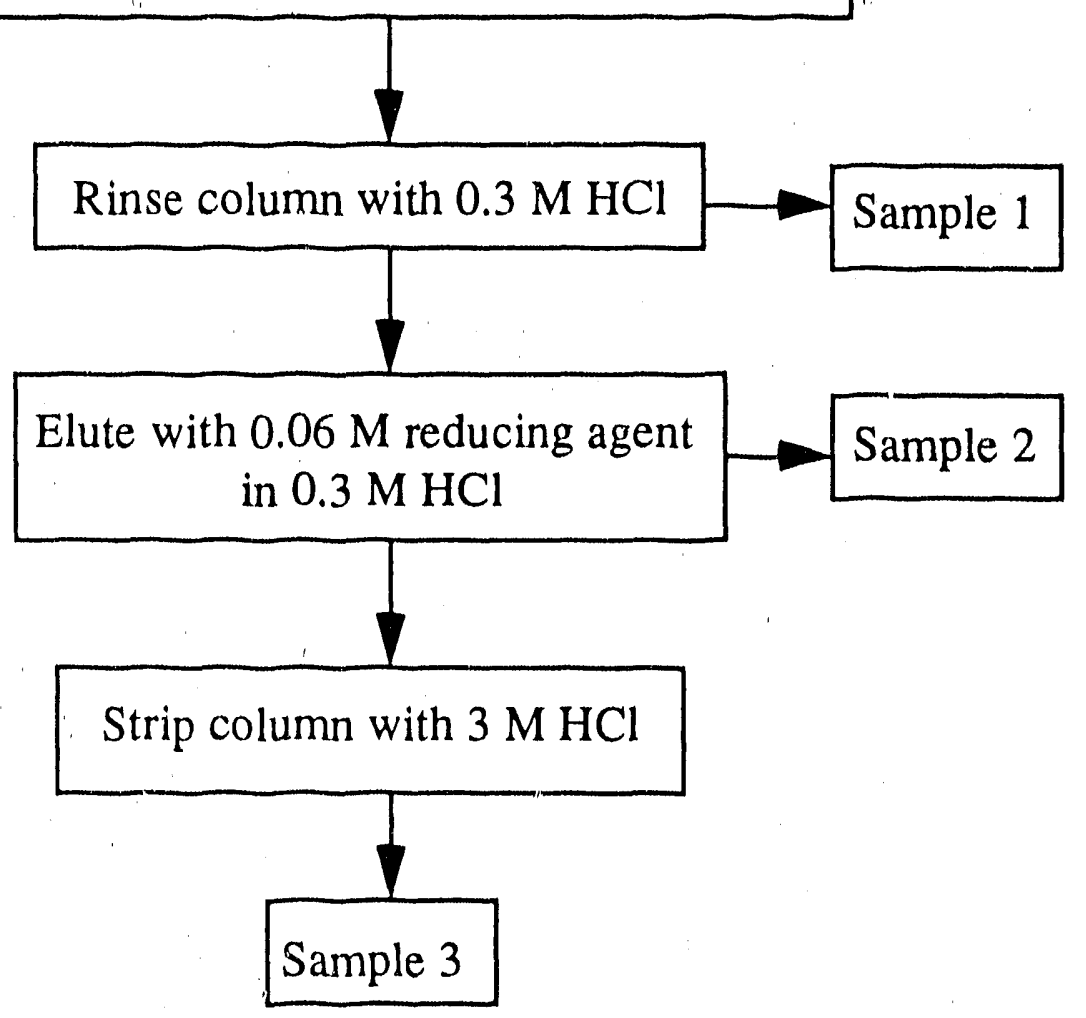

Figure 23: Flowchart of the HDEHP reduction procedure for $\mathrm{Lr}$ 
Hydroxylamine is a kinetically slow reducing agent due to the necessity of the molecular rearrangement. Therefore, the rapid processing time that is necessary for the experiment does not give hydroxylamine sufficient time to reduce $\mathrm{Lr}^{3+}$ to $\mathrm{L} \cdot \mathrm{r}^{1+}$.

\section{D: Non-Aqueous Experiments}

By changing the solvent from water to some organic solvent the limiting factor of the reduction of water can be removed from the systeın. Strong reducing agents are significantly more stable in non-aqueous solution. For instance, $\mathrm{SmI}_{2}$ cannot exist in aqueous solution, whereas in THF (tetrahydrofuran), the species will remain almost indefinitely as long as it is kept under an inert atmosphere. Free protons or the reduction of water itself via eq. 2 are the main limiting factors in aqueous solution.

$$
2 \mathrm{H}_{2} \mathrm{O}+2 \mathrm{e}^{-} \Leftrightarrow=\mathrm{H}_{2}+2 \mathrm{OH}^{-} \quad \mathrm{E}^{\circ}=-0.8277 \mathrm{~V} \quad \text { Eq. } 2
$$

The single largest advantage of choosing non-aqueous solvents for reduction procedures is that they can be chosen to not have protons which could be easily reduced, i.e., organic acids and alcohols. This still leaves a large selection of organic solvents in which to look for ideal properties to design an appropriate experiment for the reduction of $\mathrm{Lr}^{3+}$.

Adapting the HDEHP reverse-phase extraction procedure for use as a non-aqueous procedure seems to hold the most promise for a reduction experiment. The HDEHP procedure has advantages for this type of experiment in that ions of lower charge states can be remoyed sooner than ions with higher charge states. This is especially appreciated for this experiment due to the short half-life of ${ }^{260} \mathrm{Lr}$. This column extraction experiment can be carried out when an appropriate solvent has been found which must do three things. The first is it must not remove the HDEHP from the resin support. The second is that the solvent must not be reduced by the reducing species. The third is that the 
reducing agent and the aridyte must be soluble in the solvent. Ideally, the solvent should have characteristics similar to water, but without the reducible protons.

Propylene carbonate is one such solvent. It has a density of $1.189 \mathrm{~g} / \mathrm{ml}$, and a boiling point of $240^{\circ} \mathrm{C}$. HDEHP was found not to be soluble in propylene carbonate, which means that resin coated with HDEHP will not have the HDEHP stripped off of it upon passage of propylene carbonate through the column. Propylene carbonate does not have any alcoholic or acidic protons, and is thus considerably more resistant to strong reducing agents "han is wuter.

\section{D1: Column Procedure}

$\mathrm{Sm}^{2+}$, obtained from Morton Thiokol's Alfa Products Division, was used as the reducing agent. The $\mathrm{Sm}^{2+}$ was received as $0.1 \mathrm{M} \mathrm{SmI}_{2}$ dissolved in aninydrous THF, tored under Ar. A solution of $0.001 \mathrm{M} \mathrm{SmI}_{2}$ in propylene carbonate was prepared by adding one $\mathrm{ml}$ of the $\mathrm{Sm}$ stock solution to $99 \mathrm{ml}$ of propylene carbonate. Prior to use, the propylene carbonate was vacuum distilled from $\mathrm{Na}$ to remove any traces of water, which would instantaneously oxidize the $\mathrm{Sm}^{2+}$. The solution was prepared inside an inert atmosphere box under an Ar atmosphere. A specially made bottle was used to transfer the solution from the box to ACCESS, where it was hooked to one of the LDC pumps. A small pressure of Ar was kept inside the bottle to keep atmospheric $\mathrm{O}_{2}$ from oxidizing the $\mathrm{Sm}^{2+}$.

An HDEHP column was prepared by adsorbing HDEH? from acetone onto a hydrophobic celite support. The resin was packed into a glass column that was $2 \mathrm{~mm}$ i.d. by $25 \mathrm{~mm}$ length. The column was held in a specially constructed Al heater block in the ACCESS equipment rack. For this set of experiments, the column was kept at ambient

temperature. $\mathrm{Sm}^{2+}$ is a fast reducing agent, since only an electron transfer is required, and not a molecular rearrangement, as is required for hydroxylamine. It is therefore not 
necessary to heat the column for kinetic reasons.

Recoiling product atoms from the ${ }^{249} \mathrm{Bk}+18 \mathrm{O}$ reaction were transported via the $\mathrm{He} / \mathrm{KCl}$ aerosol jet to a collection site on top of the experimental cave. The $\mathrm{KCl}$ aerosols were collected on $\mathrm{Pt}$ foils at the end of the capillary tube. The $\mathrm{KCl}$ spot was dissolved in $0.05 \mathrm{M} \mathrm{HCl}$ and injected into the ACCESS injection lnop. The activity was then sorbed on the column from $0.05 \mathrm{M} \mathrm{HCl}$. After the activity was adsorbed onto the resin, the resin was washed with pure propylene carbonate to remove the aqueous phase. The $\mathrm{Sm}^{2+}$ solution was then pumped through the column. About 4 frec column volumes of the $\mathrm{Sm}^{2+}$ solution were pumped through the column and collected on heated Ta foils. The column was stripped using $3 \mathrm{M} \mathrm{HCl}$. This strip contains species with oxidation states greater than 1+. After evaporation of the solvent, the foils were placed on surface barrier detectors to look for $\alpha$ and SF decay.

\section{D2: Results and Discussion}

Several problems were encountered with this procedure. The first and most significant was the degradation of the reducing agent. $\mathrm{Sm}^{2+}$, in the iodide form, has a deep blue color in THF. When dissolved in propylene carbonate, the $\mathrm{Sm}^{2+}$ takes on a red color. During the chemical procedure the condition of the reducing agent can be monitored since the tubes in ACCESS are transparent. It was found that during the procedure, the propylene carbonate solution was tuining yellow before it reached the column. The yellow color indicates that the $\mathrm{Sm}$ "has oxidized to $\mathrm{Sm}^{3+}$. This might be occurring due to contact of the solution : with contaminants in the tubing. The tubing used in ACCESS is teflon, so there should not be any contamination from old solutions. Problems may also exist at the column, as propylene carbonatc may take a very long time to displace all of the water that is present from the resin. Since the experiments were performed using 3minute ${ }^{260} \mathrm{Lr}$, long processing times were avoided. Too short a flushirg period would 
result in oxidation of the $\mathrm{Sm}^{2+}$ from traces of water that remained on the column.

One other systematic problem was the increase in back pressure over time while pumping the propylene carbonate solution through the column. After a period of time, the system back pressure built up sufficiently to cause leaks at tubing connections, and finally resulted in tubing failure. The back pressure in the system had become greater than 300 psi, atier which the tubing ruptured.

An additional problem was experienced in the off-line testing of the procedure. No adequate way was found that could be used to test the procedure. The problem involves finding a species that will stick to the resin and be reducible to an oxidation state that will not stick to the resin. No such species can be found in the periodic table, short of the possibility of the $3+/ 1+$ oxidation state couple occurring in Lr. Additionally, it would be desirable to ensure that $1+$ species will be eluted from the resin using propylene carbonate. This cannot be tested using 1+ cations using the experimental procedure, as these cations will not stick to an HDEHP column from an acidic solution. It was also found to be quite difficult to dissolve evaporated tracers, such as ${ }^{137} \mathrm{Cs}$, in propylene carbonate.

For these reasons, the studies into the lower oxidation state of $\mathrm{Lr}$ using non-aqueous chemiral techniques were discontinued. 


\section{Appendix B: Attempts to Measure the Ionic Radius of $\mathrm{No}^{3+}$}

\section{A: Introduction}

The measurement of the ionic radius of the $\mathrm{No}^{3+}$ ion is of key interest, particularly since the ionic radius of the $\mathrm{Lr}^{3+}$ ion has been determined. This measurement would conclude the ionic radius determinations of the $3+$ actinide ions. In the last four elements of the lanthanide series there is an average spacing of $0.012 \mathrm{~nm}$ between adjacent elements [TEM54]. The spacing between $\mathrm{Md}^{3+}$ and $\mathrm{Lr}^{3+}$ has been determined to be $0.0013 \mathrm{~nm}$. Thus the crystal ionic radius spacing between the $\mathrm{Md}^{3+}$ ion and the $\mathrm{Lr}^{3+}$ ion corresponds to a spacing in the lanthanide series of only one $\mathrm{Z}$, whereas $\mathrm{Md}$ and $\mathrm{Lr}$ are $2 Z$ apart. The $\mathrm{No}^{3+}$ ion appears to have been squeezed out! Comparison of the end of the actinide series and the lanthanide series in terms of their relative $3+$ ionic radii becomes very interesting indeed.

The relative spacing between the other heavy members of the $3+$ actinide series is shown in table III. Of particular interest in this table is the small spacing between $\mathrm{Lr}^{3+}$ and $\mathrm{Md}^{3+}$ that was noted above. Compared to theoretical estimates, this spacing is quite small. The estimates by Bratsch and Lagowski [BRA86] seem to be the best values based on the smooth difference between neighboring atoms. This is expected from the way that they estimated their radii, as the values were scaled from the lanthanide radius values of Templeton and Dauben [TEM54]. The values from David [DAV85] show irregular spacing between each of the neighboring actinides from Am through $\mathrm{Lr}$, which does not compare well with the few experimental values that are available. Both of these theoretical estimates show that the radii should be fairly smoothly decreasing with increasing $\mathrm{Z}$. For comparison, it would then be very important to determine an 
experimental value for the ionic radius of $\mathrm{No}^{3+}$.

The ground-state electronic configuration of nobelium is $[R n] 5 f^{14} 7 s^{2}$. The $5 f$ shell forms a stronger inert core when compared to the $4 \mathrm{f}$ shell in the lanthanide series, borne out by the fact that the rare-earth homolog of nobelium, ytterbium, exists in the $3+$ oxidation state in aqueous solution. $\mathrm{Yb}$ does have an accessible $2+$ oxidation state, with a standard reduction potential of $\mathrm{E}^{\circ}=-0.578 \mathrm{~V}$ [LAT52]. Nobelium, on the other hand, exists in aqueous solution as a stable $2+$ ion, with its $5 \mathrm{f}^{14}$ electron shell remaining intact. The standard reduction potential of the $\mathrm{No}^{3+} / \mathrm{No}^{2+}$ couple was determined to be $\mathrm{E}^{\circ}=$ $1.4 \pm 0.2 \mathrm{~V}[$ SIL69].

This facet of nobelium chemistry provides special problems for the determination of the $3+$ crystal ionic radius. First is the problem of getting the $\mathrm{No}^{2+}$ ion oxidized to the $\mathrm{No}^{3+}$ oxidation state. This can be accomplished with the use of a strong oxidizing agent, such as $\mathrm{H}_{5} \mathrm{IO}_{6}, \mathrm{E}^{\circ}=1.7 \mathrm{~V}$. The second problem is to keep it in this oxidation state during the determination experiment. The classical methods for the determination of ionic radii are either $\mathrm{X}$ - ray diffraction or electron diffraction. However, these techniques require weighable quantities of a compound for the determination. In the case of nubelium, the maximum amount of material available is on the order of a few hundred atoms, which would decay with a 58 minute half-life if all of these atoms were ${ }^{259}$ No. This would give a sample that is much to small for analysis, as well as being too radioactive. Additionally, most of the sample would be lost to radioactive decay during sample preparation. Therefore, indirect methods such as the elution of the species from a cation-exchange resin column with $\alpha$-HIB must be used for this determination.

The system that was used in the attempt to determine the $\mathrm{No}^{3+}$ ionic radius was the same $\alpha$-HIB system that w'as used successfully in the $\mathrm{Lr}^{3+}$ ionic radius determination. This system required some modification, however, in order to keep the normally $\mathrm{No}^{2+}$ ion in the $\mathrm{No}^{3+}$ oxidation state. A small amount of a holding oxidant was added to the $\alpha$ $\mathrm{HIB}$ solution with the intention of keeping the $\mathrm{No}^{3+}$ in this oxidation state. $\mathrm{KMnO}_{4}$ has a 
large enough potential, $\mathrm{E}^{\circ}=1.679 \mathrm{~V}$, to retain No in the $3+$ oxidation state. The solution that is used to dissolve the $\mathrm{KCl}$ deposit from the He-jet collection site should also be an oxidizing solution, in order to initially oxidize the $\mathrm{No}^{2+}$ to $\mathrm{No}^{3+} . \mathrm{H}_{5} \mathrm{IO}_{6}$ cannot be used as the stabilizing agent, it s necessary to have the undissociated $\mathrm{H}_{5} \mathrm{IO}_{6}$ molecule present in solution along with the of all of the species are calculated for a solution that will have a $0.05 \mathrm{M} \alpha$-HIB anion concentration with a $0.05 \mathrm{H}_{5} \mathrm{IO}_{6}$ concentration, it would require a total $\alpha$-HIB concentration of $33.6 \mathrm{M}$. If an $\alpha$-HIB solution could be made this concentrated, it would be far too viscous for any practical use.

\section{B: Chemical Procedure}

The isotope of nobelium used for this study was $255 \mathrm{No}$, which is an alpha emitter with a 3.1 minute half-life. It was produced by the ${ }^{248} \mathrm{Cm}\left({ }^{16} \mathrm{O}, \alpha 5 \mathrm{n}\right)$ reaction. The target consisted of $742 \mu \mathrm{g} / \mathrm{cm}^{2}$ of ${ }^{248} \mathrm{Cm}$ electroplated in a $6-\mathrm{mm}$ diameter spot on a 2.089 $\mathrm{mg} / \mathrm{cm}^{2}$ Be backing foil. ${ }^{16} \mathrm{O}^{5+}$ beams of typically 0.5 particle $\mu \mathrm{A}$ were provided by the 88 inch cyclotron of the Lawrence Berkeley Laboratory. Recoiling product atoms were transported via a $\mathrm{He} / \mathrm{KCl}$ aerosol gas jet transport system to a collection site above the cave at the cyclotron.

The $\mathrm{KCl}$ deposit from the $\mathrm{He} / \mathrm{KCl}$ jet was dissolved after an appropriate collection time with a small volume of the oxidizing agent, typically $0.25 \mathrm{M} \mathrm{H}_{5} \mathrm{HO}_{6}$. The solution was then picked up in a transfer pipette and placed on the top of a cation exchange resin bed. The column was $5 \mathrm{~cm} \times 0.2 \mathrm{~cm}$ in size, packed with Benson BC-X12 strongly acidic cation exchange resin, $12 \mu \mathrm{m}$ particle size. The resin had been pretreated with $1 \mathrm{M}$ $\mathrm{NH}_{4} \mathrm{Cl}$ to convert it from the $\mathrm{H}^{+}$form to the $\mathrm{NH}_{4}{ }^{+}$form. The solution was pushed onto the resin using about $10 \mathrm{psi}$ of nitrogen. Next a small volume of water was passed through the resin to remove all of the $\mathrm{HCl}$ that was present. Finally the eluting agent, $\alpha$ - 
$\mathrm{HIB}$, adjusted to an appropriate $\mathrm{pH}$ for the experiment, was passed through the column. Since it was necessary to measure the relative elution positions of Lr-No, No-Md, and Md-Fm under these conditions, solutions of differing $\alpha$-HIB anion concentration were used so that activities could be eluted from the column in an appropriate amount of time for detecting the desired isotopes. Fractions from the column were collected dropwise on $\mathrm{Ta}$ foils. After the eluant was evaporated to dryness, the foils were flamed and then placed on silicon surface barrier detectors for alpha and spontaneous fission counting.

The data were collected and stored on magnetic tape using the RAGS system. The data were stored in event mode, with every event tagged with the time that it occurred. The data is also displayed on the computer terminal, so that the experiment can be monitored while in progress. The final analysis was performed off-line.

\section{C: Results and Discussion}

The most promising option for this procedure was to use $\mathrm{KMnO}_{4}$ as the stabilizing agent. Its presence in solution will not aiter the $\mathrm{pH}$ of the $\alpha$-HIB solution, which is critical for getting reproducible results. Changing the $\mathrm{pH}$ of the solution will change the $\alpha$-HIB anion concentration, and thereby alter the elution positions of the elements of interest from the column.

Several different conditions were tried for this experiment. The first set of experiments was performed using both $\mathrm{H}_{5} \mathrm{IO}_{6}$ and $\mathrm{KMnO}_{4}$, as a preoxidizer and stabilizing agent, respectively. A second set was performed using only $\mathrm{H}_{5} \mathrm{IO}_{6}$ to oxidize the $\mathrm{No}^{2+}$ prior to putting it onto the resin bed, without any stabilizing agent in the $\alpha$-HIB solution. The third set of experiments did not have any $\mathrm{H}_{5} \mathrm{IO}_{6}$ present, and only used the $\mathrm{KMnO}_{4}$ in the $\alpha$-HIB solution for the oxidation of $\mathrm{No}^{2+}$. The results of these three sets of conditions are summarized in table IV. A total of 42 separations was performed using these conditions. 
One of the largest problems encountered with this procedure was the shifting elution position of Fm. The other elements in the elution were shifting also, but Fm is the easiest element to detect in these experiments due to its large production tate and relatively short half-life. The shifting of the elution position makes the determination of precise relative elution positions impossible, as many separations need to be performed in order to accurately determine the position of $\mathrm{Lr}$ and No due to their low detection rate.

A second problem was the degradation of the $\alpha$-HIB solutions containing $\mathrm{KMnO}_{4}$. They were found to degrade fairly quickly at ambient temperature, and very rapidly when placed on a heated resin column. The column was run at room temperature in these experiments to alleviate this problem. The degradation was noted by the replacement of the normal purple $\mathrm{KMnO}_{4}$ color with the brown color of $\mathrm{MnO}_{2}$, the reduction product of $\mathrm{KMnO}_{4}$. This brown solution eventually produced a black precipitate on the bottom of the container, an additional confirmation that the $\mathrm{KMnO}_{4}$ species had been reduced. The most probable explanation for this is the oxidation of the alpha-hydroxyl group of the $\alpha$ HIB to an aldehyde. This type of reaction is normally carried out using $\mathrm{Na}_{2} \mathrm{Cr}_{2} \mathrm{O}_{7}$ as the oxidizing agent, however $\mathrm{KMnO}_{4}$ should be able to oxidize primary alcohol species as well. This effect also explains the shifting elution positions, as the older the $\alpha$-HIB solution is, the greater the extent of oxidation of the elutriant, thereby lowering its concentration and delaying the elution of the elements from the column.

It might be possible to perform this experiment by eluting the actinide elements from an HDEHP column using an oxidizing species. Elutions from an HDEHP column are frequently performed using $\mathrm{HNO}_{3}$ as the eluant, and are therefore performed under oxidizing conditions. Addition of a stabilizing agent should not have a large effect on the elution if its concentration can be kept low enough.

There are a couple of significant problems with this proposal. The first is that the elements will be eluted in order of increasing $\mathrm{Z}$, which is less than ideal from a contamination standpoint. In the transfer reactions that are used to produce the nuclides 
for study in these experiments, elements that are closest to the projectile $\mathrm{Z}$ are produced in the largest quantities. See for example [LEE83]. This will be a problem in that the elements that have higher production cross sections will be eluted from the resin first. The normal tails of the elution peaks will result in contamination of the elemental fractions with lower production rates which come after the fractions with higher production rates. This will cause problems trying to find precise elution positions by looking at specific isotopes that have similar decay properties. The order of elution of elements from this column is itself a problem in that the elements having the isotopes. with the shortest half-lives will be eluted from the column last, e.g., No and $\mathrm{Lr}$ isotopes. Therefore, an elution that is appropriate for the separation of Es and Fm cannot be used for the separation of No and Lr, simply because it would take too long.

Another problem with this procedure involves the tetrad effect. As can be seen in figure 24, the plot of ionic radius versus elution position from an HDEHP column does not give a straight line. Instead, it shows the well established tetrad effect for the lanthanide and actinide ions [HOR59][HOR69A][NUG70][PEP60][PEP70]. The main problem here is that in this system elements that have the same elution position do not have the same radius. The shape of the tetrad curve in the No region makes the expected elution position nearly the same as $\mathrm{Lr}$. This creates an additional problem in that ${ }^{255} \mathrm{No}$ is the best isotope to use for the determination of the No elution position, while ${ }^{260} \mathrm{Lr}$, which has very similar decay properties, is the isotope that is best suited for the determination of the Lr elution position.

From figure 25, it becomes apparent that the ionic radius of the $\mathrm{No}^{3+}$ ion cannot be determined using an HDEHP experiment, as there is no way of unfolding the tetrad effect from the elution position data. 


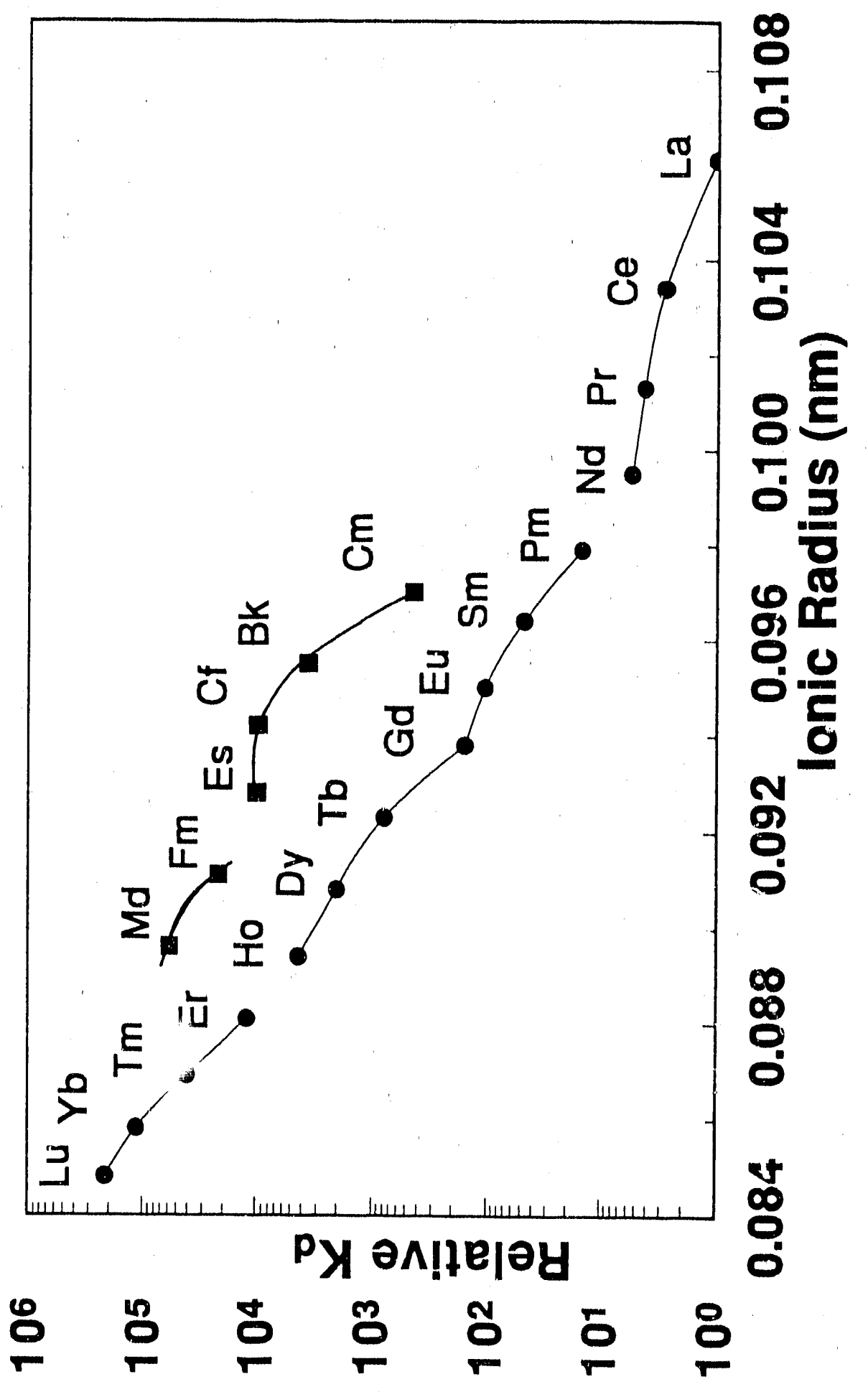

Figure 24: Relative distribution coefficients vs. $3+$ crystal ionic radii for elution from an HDEHP column with $\mathrm{HNO}_{3}$ 


\section{REFERENCES}

[AHR76] H. Ahrens, N. Kaffrell, N. Trautmann, G. Herrmann, Phys. Rew. C 14(1) 211 (1976)

[AUM74] D.C. Aumann, G. Müllen, Nucl. Instr. Meth. 75,115 (1974)

[BEM76] C.E. Bemis, D.C. Hensley, P.F. Dittner, R.L.Hahn, R.J. Silva, J.K.Tarrant, L.D. Hunt, ORNL-5137, 73 (1976)

[BEM77] C.E. Bemis, P.F. Dittner, R.J. Silva, R.L. Hahn, J.R. Tarrant, L.D. Hunt, D.C. Hensley, Dhys. Rev. C 16(3), 1146 (1977)

[BEM77A] C.E. Bemis, R.L. Ferguson, R.J. Silva, F. Plasil, G.G. O'Kelley, R.L. Hahn, D.C. Hensley, E.K. Hulet, R.W. Lougheed, Bull. Am. Phys. Soc. 22(4), 611 (1977)

[BOR81] R.J. Borg, G.J. Dienes, J. Inorg. Nucl. Chem. 43, 1129 (1981)

[BRA86] S.G. Bratsch and J.J. Lagowski, J. Phys. Chem. 20, 307 (1986)

[BRE84] L. Brewer, High Temp. Sci. 111, 1 (1984)

[BRU88] W. B 'le, M. Schädel, U.W. Scherer, J.V. Kratz, K.E. Gregorich, D. Lee, M. Nurmia, R.M. Chasteler, H.L. Hall, R.A. Henderson, D.C. Hoffman, Inorg. Chim. Acta 146, 267 (1988)

[BUS59] Yu.A. Buslaev and N.S. Nikolaev, Russ. J. Inorg. Chem. 4(2), 210 (1959)

[CHO56] G.R. Choppin and R.J. Silva, J. Inorg. Nucl. Chem. 3, 153 (1956)

[CHO61] G.R. Choppin and J.A. Chopoorian, J. Inorg. Nucl. Chem. 22, 97 (1961)

[DAV85] F. David, B. Fourest, J. Duplessis, J. Nucl. Mat. 130, 273 (1985)

[DES73] J.P. Desclaux, At. Data Nucl. Data 12, 311 (1973)

[DES80] J.P. Desclaux, B. Fricke, J. Phys. 41, 943 (1980)

[DON65] E.D. Donets, V.A. Shchegolev, V.A. Ermakov, Atomnaya Énergiya 19(2), 109 (1965)

[DRU70] V.A. Druin, Yadern. Fiz. 12, 268 (1970) 
[ESK71] K. Eskola, P. Eskola, M. Nurmia, A. Ghiorso, Phys. Rev. C 4(2), 632 (1971)

[ESK 73] P. Eskola, Phys. Rev. C Z(1), 280 (1973)

[EVA72] J.E. Evans, R.W. Lougheed, M.S. Coops, R.W. Hoff, E.K. Hulet, Nucl. Instr. Meth. 102, 389 (1972)

[FLE68] G.N. Flerov, V.A. Druin, A.G. Demin, Yu.V. Lobanov, N.K. Skobelev, G.N. Akapiev, B.V. Fefilov, I.V. Kolesov, K.A. Gavrilov, Yu.P. Kharitonov, L.P. Chelnokov, Joint Institute of Nuclear Research, Dubna, preprint P7-3808 (1968)

[FLE70] G.N. Flerov, Yu.Ts. Oganessian, Yu.V. Lobanov, Yu.A. Lazarev, S.P. Tretiakova, I.V. Kolesov, V.M. Plotko, Atomnaya Énergiya 22, 243 (1970)

[FLE76] G.N. Flerov, Proceedings of the Third International Conference on Nuclei Ear From Stability Cargèse Corsica. France 542 (1976)

[GHI61] A. Ghiorso, T. Sikkeland, A.E. Larsh, R.M. Latimer, Phys. Rev. Lett. 6, $473(1961)$

[GHI70] A. Ghiorso, M. Nurmia, K. Eskola, J. Harris, P. Eskola,Phys. Rev. Lett. 24, $1498(1970)$

[GHI70A] A. Ghiorso, M.Nurmia, K. Eskola, P. Eskola, Phys. Lett. 32B, 95 (1970)

[GHI71] A. Ghiorso, M. Nurmia, K. Eskola, P. Eskola, Phys. Rev. C 4(5), 1850 (1971)

[GLE8 8 ] V.A. Glebov, L. Kasztura, V.S. Nefedov, B.L. Zhuikov, Radiochimica Acta 46, 117 (1989)

[GRE90] K.E. Gregorich, submitted to Nucl.Inst. Methods (1990)

[HA173] R.G. Haire and R.D. Baybarz, J. Inorg. Nucl. Chem. 35, 489 (1973)

[HAL89] H.L. Hall, M.J. Nurmia and D.C. Hoffman, Nucl. Instr. Meth. Phys. Res. A276, 649 (1989)

[HES85] F.P. Hessberger, G. Münzenberg, S. Hofmann, Y.K. Agarwal, K. Poppensieker, W. Reisdorf, K.-H. Schmidt, J.R.H. Schneider, W.F.W. Schneider, H.J. Schött, P. Armbruster, B. Thuma, C.-C. Sahm, D. Vermeulen, Z. Phys. A 322, 557 (1985) 
[HOF87] D.C. Hoffman, D.M. Lee, C.M. Gannett, R.A. Henderson, R.W. Lougheed, E.K. Hulet, J.F. Wild, K.J. Moody, R.J. Dougan, LBL Nucl. Sci. Div. Ann. Rep., 1986-1987, LBL-25295

[HOF87A] D.C. Hoffman, L.P. Somerville, Lawrence Berkeley Laboratory report LBL-23475

[HOF89] D.C. Hoffman, Nucl. Phys.A502, 21c (1989)

[HOR69] E.P. Horowitz, C.A.A. Bloomquist, D.J. Henderson, J. Inorg. Nucl. Chem. 31, 1149 (1969)

[HOR69A] E.P. Horowitz, C.A.A. Bloomquist, D.J. Henderson, D.E. Nelson, J. Inorg. Nucl. Chem.31, 3255 (1969)

[HUL83] E.K. Hulet, Radiochimica Acta 32, 7 (1983)

[HUL84] E.K. Hulet, LLNL Nucl, Chem, Div Ann, Rep, 6-19 (1984)

[HYD87] E.K. Hyde, D.C. Hoffman, O.L. Keller, Radiochimica Acta 4(2), 57 (1987)

[KEL63] O.L. Keller, Jr., Inorg. Chem. 2, 783 (1963)

[KEL66] O.L. Keller, Jr., A. Chetham-Strode, Jr. Inorg. Chem. 5, 367 (1966)

[KEL77] O.L. Keller, Jr., G.T. Seaborg, Ann.Rev. Nucl. Sci. 27, 139 (1977)

[KEL84] O.L. Keller, Jr., Radiochimica Acta 37, 169 (1984)

[LAT52] W.M. Latimer, Oxidation Potentials, Prentice Hall, Inc., New York, (1952)

[LED78] C.M. Lederer and V.S. Shirley, eds., Table of Iso+opes, seventh edition, Wiley Interscience, New York, ap.-19, (1978)

[LEE83] D. Lee, K.J. Moody, M.J. Nurmia, G.T. Seaborg, H.R. von Gunten, D.C. Hoffman, Phys. Rev. C 27(6), 2656 (1983)

[MAR69] Y. Marcus, A.S. Kertes, Ion Exchange and Solvent Extraction of Metal Complexes, Wiley Interscience, New York, p. 287 (1969)

[MAR69A] Y. Marcus, A.S. Kertes, ibid, p. 634-45 (1969)

[MIK89] N.B. Mikheev and A.N. Kamenskaya, Radiokhimiya 31(1), 95 (1989)

[MIL55] G.W.C. Milner, G.A. Barnett, A.A. Smales, Analyst 80,380 (1955) 
[MO083] K.J. Moody, Actinide Prodluction in the Reaction of Heavy Ions With Curium-248, PhD Thesis, LBL-16249 (1983)

[MUL75] G. Müllen, D.C. Aumann, Nucl. Instr. Meth. 128, 425 (1975)

[MUN81] G. Munzenberg, S. Hofmann, F.P. Hessberger, W. Reisdorf, K.H. Schmidt, J.H.R.Schneider, P. Armbruster, C.C. Sahm, B. Thuma, Z. Phys. A30Q, 107 (1981)

[NIK59] N.S. Nikolaev and Yu.A. Buslaev, Russ. J. Inorg. Chem. 4.(1), 84 (1959)

[NOR70] L.C. Northcliff and R.F. Schilling, Nucl. Data Tables Z(3-4), 235 (1970)

[NUG70] L.J. Nugent, J. Inorg. Nucl. Chem. 32, 3485 (1970)

[OGA76] Yu.Ts. Oganessian, A.G. Demin, N.A. Danilov, G.N. Flerov, M.P. Ivanov, A.S. Iljinov, N.N. Kolesnikov, B.N. Markov, V.M. Plotko, S.P. Tretyakova, Nucl. Phys. A273, 505 (1976)

[OGA83] Yu.Ts. Oganessian, IINR-D7-83-644 (1983)

[PAL88] C.E.A. Palmer, H.L. Hall, P.A. Baisden, D.C. Hoffman, D.M. Lee, Lawrence Livermore Laboratory Nuclear Chemistry Division Annual Report, UCRL- ,126 (1988)

[PEP60] D.F. Peppard, G.W. Mason, S. Lewey, J. Inorg. Nucl. Chem.31, 2271 (1960)

[PEP70] D.F. Peppard, C.A.A. Bloomquist, E.P. Horowitz, S. Lewey, G.W. Mason, J. Inorg. Nucl. Chem. 32, 339 (1970)

[PHI66] C.S.G. Phillips, R.J.P. Williams, Inorganic Chemistry Vol.2, Oxford

University Press, New York, p. 58 (1966)

[PIT79] K.S. Pitzer, Acc. Chem. Res. 12, 271 (1979)

[PYY79] P. Pyykko, J. Desclaux, Acc. Chem. Res.12, 276 (1979)

[PYY88] P. Pyykko, Chem.Rev.88, 563 (1988)

[SEA45] G.T. Seaborg, Chem. Eng. News 23, 2190 (1945)

[SIL69] R.J. Silva, T. Sikkeland, M. Nurmin, A. Ghiorso, J. Inorg. Nucl. Chem. 31, 3405 (1969)

[SIL70] R.J. Silva, T. Sikkeland, M. Nurmia, A. Ghiorso, Inorg. Nl l. Chem. Lett. 6. $733(1970)$ 
[SIL.70A] R.J. Silva, J. Harris, M. Nurmia, K. Eskola, A. Ghiorso, Inorg. Nucl.

Chem. Lett. 6, 871 (1970)

[SMI56] H.L. Smith, D.C. Hoffman, J. Inorg. Nucl. Chem. 3, 243 (1956)

[SOM85] L.P. Somerville, M.J. Nurmia, J.M. Nitschke, A. Ghiorso, Phys. RevC31, 1801 (1985)

[STE53] P.C. Stevenson, H.G. Hicks, Anal. Chem. 25, 1517 (1953)

[STR67] V.M. Strutinsky, Nucl. Phys. A95, 420 (1967)

[TEM54] D.H. Templeton and C.H. Dauben, J. Am. Chem. Soc. 76, 5237 (1954)

[TRA76] N. Trautmann, G. Herrmann, J. Radioanal. Chem. 32, 533 (1976)

[UNI74] J.P. Unik, J.E. Gindler, L.E. Glendenin, K.F. Flynn, A. Gorski, R.K. Sjoblom, Proceedings of the Third International IAEA Symposium on the Physics and Chemistry of Fission, Rochester, 1973 (IAEA, Vienna), Vol. II., 33 (1974)

[VIO66] V.E. Viola, Nucl. Data A1, 391 (1966)

[WAT57] G.R. Waterbury and C.E. Bricker, Anal. Chem. 29(10), 1474 (1957)

[WEI81] M. Weis and H.O. Denschlag, J. Inorg. Nucl. Chem. 43, 437 (1981)

[WER54] J.R. Werning, K.B. Higbie, J.T. Grace, B.F. Speece, H.L. Gilbert, Ind. Eng. Chem. 46, 644 (1954)

[ZVA66] I. Zvara, Yu.T. Chuburkov, R. Caletka, T.S. Zvarova, M.R. Shalaevsky, B.V. Shilov, Atomn. Energ. 21, 83 (1966)

[ZVA69] I. Zvara, Yu.T. Chuburkov, R. Caletka, M.R. Shalaevsky, Radiokhimiya 11, 163 (1969)

[ZVA70] I. Zvara, Yu.T. Chuburkov, V.Z. Belov, G.V. Buklanov, B.B. Zakhvatev, T.S. Zvarova, O.D. Maslov, R. Caletka, M.R. Shalaevsky, J. Inorg. Nucl. Chem. 32, 1885 (1970)

[ZVA74] I. Zvara, B. Eichler, V.Z. Belov, T.S. Zvarova, Yu.S. Korotkin, M.R. Shalaevskii, V.A. Schegolev, M. Hussonnois, Sov. Radiochem. 16, 709 (1974)

[ZVA76] I. Zvara, V.Z. Belov, V.P. Domanov, M.R. Shaldevskii, Sov. Radiochem. 18, 328 (1976) 

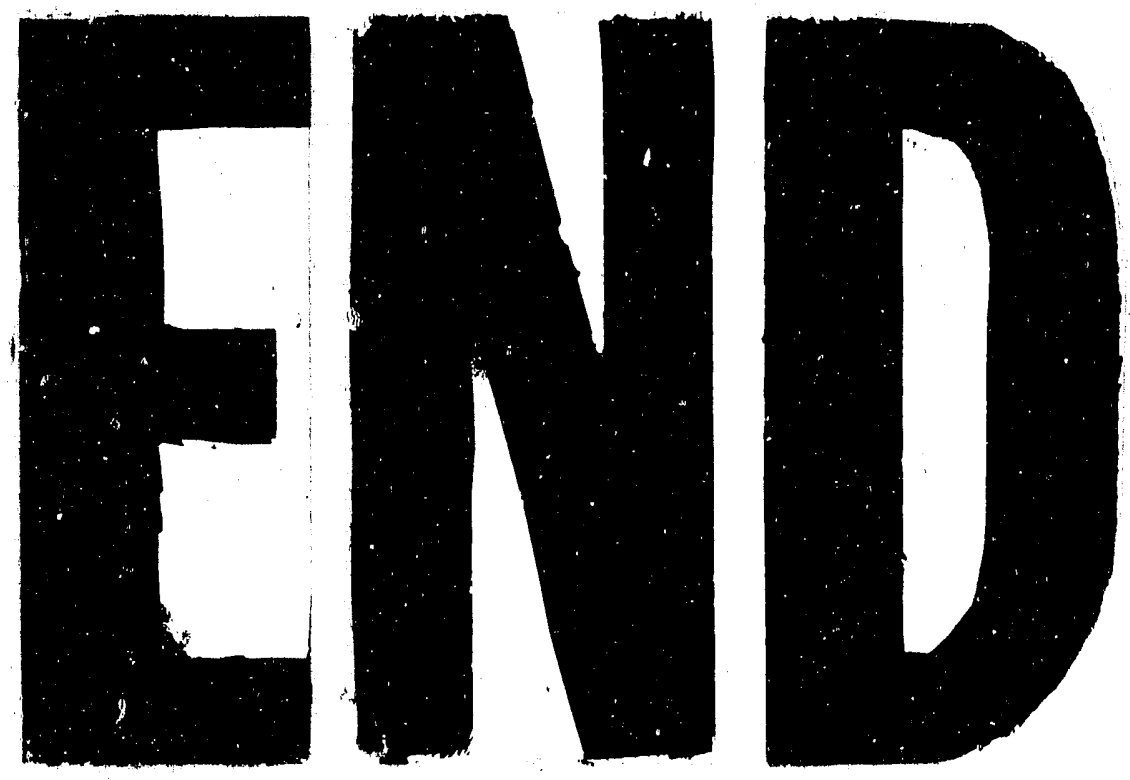

,
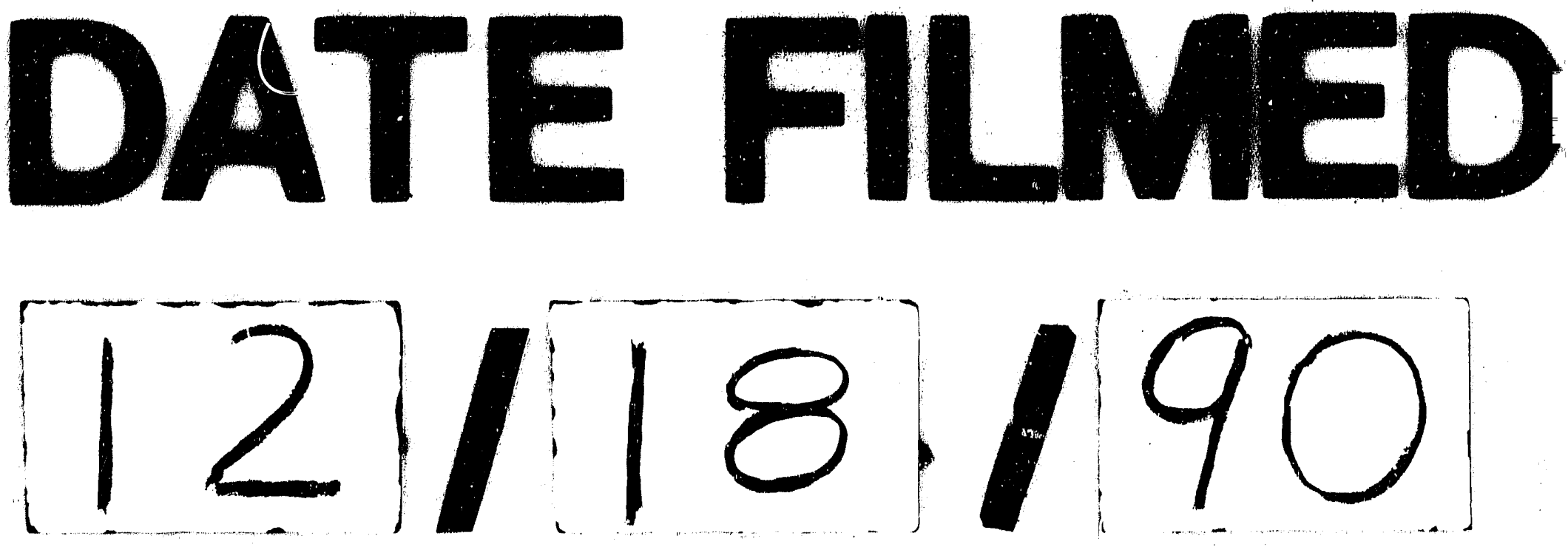
